\title{
A REDUCTION THEOREM FOR THE MCKAY CONJECTURE
}

\author{
by \\ I. M. Isaacs \\ Mathematics Department \\ University of Wisconsin \\ 480 Lincoln Drive \\ Madison WI 53706 USA \\ E-mail: isaacs@math.wisc.edu \\ Gunter Malle \\ Fachbereich Mathematik \\ Universität Kaiserslautern \\ Postfach 3049 \\ 67653 Kaiserslautern GERMANY \\ E-mail:malle@mathematik.uni-kl.de \\ Gabriel Navarro \\ Departament d'Àlgebra \\ Facultat de Matemàtiques \\ Universitat de València \\ 46100 Burjassot. València SPAIN \\ E-mail: gabriel@uv.es
}

\begin{abstract}
The McKay conjecture asserts that for every finite group $G$ and every prime $p$, the number of irreducible characters of $G$ having $p^{\prime}$-degree is equal to the number of such characters of the normalizer of a Sylow $p$-subgroup of $G$. Although this has been confirmed for large numbers of groups, including, for example, all solvable groups and all symmetric groups, no general proof has yet been found. In this paper, we reduce the McKay conjecture to a question about simple groups. We give a list of conditions that we hope all simple groups will satisfy, and we show that the McKay conjecture will hold for a finite group $G$ if every simple group involved in $G$ satisfies these conditions. Also, we establish that our conditions are satisfied for the simple groups $\mathrm{PSL}_{2}(q)$ for all prime powers $q \geq 4$, and for the Suzuki groups $\mathrm{Sz}(q)$ and Ree groups $\mathrm{R}(q)$, where $q=2^{e}$ or $q=3^{e}$ respectively, and $e>1$ is odd. Since our conditions are also satisfied by the sporadic simple group $J_{1}$, it follows that the McKay conjecture holds (for all primes $p$ ) for every finite group having an abelian Sylow 2-subgroup.
\end{abstract}

The third author is partially supported by the Ministerio de Educación y Ciencia proyecto MTM2004-06067-C02-01. 


\section{Introduction.}

As usual, we write $\operatorname{Irr}(G)$ to denote the set of irreducible (complex) characters of a finite group $G$. Also, if $p$ is a prime number, we write $\operatorname{Irr}_{p^{\prime}}(G)$ for the set of those characters in $\operatorname{Irr}(G)$ whose degree is not divisible by $p$. The well known McKay conjecture asserts that if $G$ is an arbitrary finite group and $p$ is any prime, then $\left|\operatorname{Irr}_{p^{\prime}}(G)\right|=\left|\operatorname{Irr}_{p^{\prime}}(N)\right|$, where $N$ is the normalizer in $G$ of a Sylow $p$-subgroup of $G$.

The McKay conjecture is known to be true for all solvable groups (for all primes) and for all $p$-solvable groups (for the prime $p$ ). In addition, it is known to be true (for all primes) for symmetric groups, general linear groups, sporadic simple groups and various other classes of simple or almost simple groups.

Since the conjecture is completely general, it is natural to hope that some argument might exist that would prove it for all groups simultaneously, but unfortunately, no one seems to have an inkling of how such a proof might proceed. Failing that, one could try to reduce the problem to a question about simple groups, which could then, in principle, be settled on a case-by-case basis. Indeed, in the early 1990s, E. C. Dade announced that he had found such a reduction.

In [3] and [4], Dade proposed a series of conjectures that relate the irreducible character degrees of a group $G$ with those of certain of its $p$-local subgroups. One of these, Dade's "projective conjecture", implies the McKay conjecture (and also much more). Dade asserted that the most "complex and delicate" of his conjectures, the "final conjecture" has the property that if it holds for all simple groups, then it automatically holds for arbitrary finite groups. Since Dade's final conjecture is a refinement of his projective conjecture, it too implies McKay. If Dade is correct, therefore, and it really does suffice to check the final conjecture for simple groups, this provides a potential route toward a proof of the McKay conjecture: check the final conjecture for all simple groups. Unfortunately, no proof seems to have been published that establishes Dade's assertion that it suffices to check the final conjecture for simple groups only. Also, it seems that checking Dade's final conjecture for simple groups is a daunting task.

For Dade's projective conjecture, however, there has been progress toward a reduction to simple groups. Although a full reduction has not been attained, G. R. Robinson and C. W. Eaton have shown that in a minimal counterexample, the Fitting subgroup would be central, and there would be a single conjugacy class of components. (See [18] and [6].)

Our goal in this paper is somewhat more modest: we present a reduction to simple groups that is specifically tailored to the McKay conjecture. Although much work remains to be done in order to establish that every nonabelian simple group satisfies our conditions for every prime, we do show that these conditions are met for all of the following simple groups: $\mathrm{PSL}_{2}(q)$, where $q$ is any prime power exceeding 3 ; the Suzuki simple groups $\mathrm{Sz}\left(2^{e}\right)$, and the Ree groups $\mathrm{R}\left(3^{e}\right)$, where in the latter two cases, of course, e must be odd and at least 3 .

Our conditions are fairly complex; they involve automorphism groups and perfect central extensions, and not just the simple group itself. (We mention the analogy with another of Dade's conjectures, his "invariant conjecture", which refines the projective conjecture to give compatibility with automorphisms.) Of course, for each isomorphism type of simple group, there are only finitely many perfect central extensions, and so there 
are just finitely many things to check.

We certainly do not claim that to establish the McKay conjecture in general, it suffices to check it for simple groups. Nevertheless, we shall see in Lemma 10.3 that if a simple group $X$ has a trivial Schur multiplier and a trivial outer automorphism group, then $X$ automatically satisfies our conditions if the McKay conjecture holds for $X$. In particular, Lemma 10.3 applies to the simple group $J_{1}$, which therefore satisfies our conditions. (It is well known that $J_{1}$ has trivial Schur multiplier and trivial outer automorphism group, and by [24], the McKay conjecture holds for all sporadic simple groups, including $J_{1}$.) Our conditions, therefore, hold for every simple group that has an abelian Sylow 2-subgroup since by [23], the only such groups are $J_{1}$, the groups $\mathrm{R}\left(3^{e}\right)$, and certain of the groups $\mathrm{PSL}_{2}(q)$. This enables us to prove the following.

THEOREM A. Let $G$ be a group with the property that each nonabelian composition factor is either of the form $\mathrm{PSL}_{2}(q)$ or is one of the Suzuki groups $\mathrm{Sz}(q)$ or Ree groups $R(q)$ mentioned above. Then the McKay conjecture holds in $G$ for all primes $p$. Also, the McKay conjecture holds for all primes $p$ for all finite groups that have an abelian Sylow 2-subgroup.

The statement of Theorem A may be somewhat misleading because in general, it is not sufficient to check composition factors. To establish the McKay conjecture for a group $G$, we require that every nonabelian simple group involved in $G$ should satisfy our conditions. (Recall that that a group $X$ is said to be "involved" in $G$ if there exist subgroups $H \triangleleft K \subseteq G$ such that $K / H \cong X$.) But each simple group involved in $G$ is involved in some composition factor of $G$, and since all nonabelian simple groups involved in any of the groups in the above list are also in the list, it is enough to check composition factors in Theorem A.

Given a prime $p$, we describe (in Section 10) a list of conditions that a nonabelian simple group $X$ of order divisible by $p$ must satisfy in order to qualify as being "good" for the prime $p$. We establish the following Theorem B, and then, once we show that the simple groups we listed previously are good for all primes that divide their orders, Theorem $\mathrm{A}$ is an immediate consequence.

THEOREM B. Fix a prime $p$ and let $G$ be a finite group. Suppose that every nonabelian simple group that is involved in $G$ and has order divisible by $p$ is good for $p$. Then the McKay conjecture holds in $G$ for the prime $p$.

Of course, Theorem B includes the fact that the McKay conjecture is valid for the prime $p$ in a $p$-solvable group, but we do not give an independent proof of this. Indeed, as we shall see, our proof of Theorem B relies on a strong form (due to T. R. Wolf) of the $p$-solvable case of the McKay conjecture.

A number of generalizations of the McKay conjecture have been proposed. The earliest of these was due to Alperin [1], who suggested a block-theoretic version. This AlperinMcKay conjecture is also a consequence of Dade's projective conjecture, and it too is is known to hold in many cases, including $p$-solvable groups. (See [17].)

More recently, Isaacs and Navarro [11] proposed a refinement of the McKay conjecture that subdivides each of the sets $\operatorname{Irr}_{p^{\prime}}(G)$ and $\operatorname{Irr}_{p^{\prime}}(N)$ into $(p-1) / 2$ subsets depending on the degrees of the characters modulo $p$. The refined conjecture then asserts that the cardinalities of the corresponding subsets of $\operatorname{Irr}_{p^{\prime}}(G)$ and $\operatorname{Irr}_{p^{\prime}}(N)$ are equal. More recently 
still, Navarro proposed [16] a version of the McKay conjecture involving Galois automorphisms, and Turull [21] has refined that to a statement involving fields of character values and Schur indices. But for the purpose of this paper, we ignore all of that and we revert to the basic McKay conjecture that we stated earlier. We would expect, however, that methods similar to those we present here might also reduce some of these refinements to questions about simple groups.

But there is one refinement of the McKay conjecture that we do not want to ignore. Indeed, it is the key to our inductive argument. Given $L \triangleleft G$ and a character $\nu \in \operatorname{Irr}(L)$, we write $\operatorname{Irr}(G \mid \nu)$ to denote the set of characters $\chi \in \operatorname{Irr}(G)$ that lie over $\nu$. (This means that $\nu$ is a constituent of the restriction $\chi_{L}$.) Of course, $\operatorname{Irr}_{p^{\prime}}(G \mid \nu)$ is the set of these characters that have $p^{\prime}$-degree.

CONJECTURE C (Relative McKay). Let $L \triangleleft G$ and fix a prime $p$. Choose $P / L \in$ $\operatorname{Syl}_{p}(G / L)$ and suppose that $\nu \in \operatorname{Irr}(L)$ is $P$-invariant. Then if $N=\mathbf{N}_{G}(P)$, we have $\left|\operatorname{Irr}_{p^{\prime}}(G \mid \nu)\right|=\left|\operatorname{Irr}_{p^{\prime}}(N \mid \nu)\right|$

Of course, we can recover the original McKay conjecture from this relative version by taking $L=1$ (and $\nu$ the principal character of $L$ ). It is known that the relative McKay conjecture is valid if $G$ is $p$-solvable. (See Theorem 1.14 of Wolf's paper [24].) Our main result extends Wolf's theorem by showing that Conjecture $\mathrm{C}$ holds if every nonabelian simple group that has order divisible by $p$ and is involved in $G / L$ is good for $p$.

In order to outline the two key steps in our argument, suppose that we are in the situation of Conjecture $\mathrm{C}$ and that all nonabelian simple groups involved in $G / L$ are good for $p$. Our first major reduction proceeds by induction on $|G / L|$. Appealing repeatedly to the inductive hypothesis, we show that we can assume $L$ is a central, cyclic $p^{\prime}$-group and that $G / L$ has a unique minimal normal subgroup $K / L$, where $K / L$ is nonabelian and has order divisible by $p$. In this situation, of course, $L=\mathbf{Z}(G)$ and $K / L$ is a direct product of isomorphic simple groups. Also, these simple factors have order divisible by $p$, and by hypothesis, they are good for $p$. In other words, the first reduction brings us to the following situation. (We mention that a result related to our first reduction has recently been published by M. Murai [15].)

HYPOTHESIS D. Let $X$ be a nonabelian simple group of order divisible by $p$, and let $\mathbf{Z}(G) \subseteq K \triangleleft G$, where $\mathbf{Z}(G)$ is a cyclic $p^{\prime}$-group and $K / \mathbf{Z}(G)$ is a direct product of isomorphic copies of $X$.

The second, and more technical step is summarized in the following theorem.

THEOREM E Fix a prime $p$ and assume Hypothesis D, where the simple group $X$ is good for $p$. Let $Q \in \operatorname{Syl}_{p}(K)$ and fix a faithful linear character $\nu$ of $\mathbf{Z}(G)$. Then there exists a subgroup $M$ of $G$ and a mapping ( )* of characters such that the following hold.

(a) $\mathbf{N}_{G}(Q) \subseteq M<G$.

(b) $\theta \mapsto \theta^{*}$ is a bijection from $\operatorname{Irr}_{p^{\prime}}(K \mid \nu)$ onto $\operatorname{Irr}_{p^{\prime}}((K \cap M) \mid \nu)$, and ( $)^{*}$ defines an isomorphism of the permutation actions of $M$ on these two sets.

(c) $\left|\operatorname{Irr}_{p^{\prime}}(G \mid \theta)\right|=\left|\operatorname{Irr}_{p^{\prime}}\left(M \mid \theta^{*}\right)\right|$ for all characters $\theta \in \operatorname{Irr}_{p^{\prime}}(K \mid \nu)$.

The organization of this paper is as follows. In Section 2, we temporarily set aside the definition of a good simple group and consider instead the class $\mathcal{X}$ of nonabelian 
simple groups $X$ (of order divisible by $p$ ) for which the conclusion of Theorem $\mathrm{E}$ is valid. (Theorem E can thus be paraphrased as "every good simple group lies in $\mathcal{X}$ ".) We then prove that Conjecture $\mathrm{C}$ holds if every nonabelian simple group involved in $G / L$ and having order divisible by $p$ lies in $\mathcal{X}$. In particular, this proves a version of Theorem $\mathrm{B}$, where the condition that the relevant simple groups are "good" is replaced by the assumption that they lie in $\mathcal{X}$. To complete the proof of Theorem B, therefore, it remains to show that all good simple groups actually do lie in $\mathcal{X}$, and this is the content of Theorem E.

In Sections 3 through 9, we develop some facts about factor sets and cohomology elements associated with invariant irreducible characters of normal subgroups of a group G. (Some of this material, especially in Section 3, is known.) In Section 10, we end the mystery and define what it means for a simple group $X$ to be good for a prime $p$. Then in Sections 11, 12 and 13, we prove a somewhat more general form of Theorem E, thereby establishing that all good simple groups are in the class $\mathcal{X}$. (The point here is that it suffices to check only finitely many things to establish that a simple group $X$ is good, while it is not clear how one might establish directly that $X$ lies in $\mathcal{X}$.) In Section 14, we prove a fairly elementary lemma about Schur multipliers of certain groups constructed from fields and Galois groups. Then finally, in Sections 15, 16 and 17, we show that the simple groups $\mathrm{PSL}_{2}(q), \mathrm{Sz}\left(2^{e}\right)$ and $\mathrm{R}\left(3^{e}\right)$ are good for all primes that divide their orders, and this establishes Theorem A.

We mention that since the final sections of this paper were written, the second author has proved that all alternating and sporadic simple groups are good for all primes, as are all simple groups of Lie type with exceptional Schur multipliers. (See [13].) Furthermore, considerable progress has been made toward proving that all simple groups of Lie type are good for all non-defining primes. (See the work of Malle [14] and B. Späth [19].)

\section{The first reduction.}

Fix a prime $p$. In this section, we assume Theorem $\mathrm{E}$ and use it to prove the relative McKay conjecture (Conjecture C) for the prime $p$. To be more precise, recall that we have defined $\mathcal{X}$ to be the class of all nonabelian simple groups of order divisible by $p$ for which the conclusion of Theorem $\mathrm{E}$ is valid. We prove the following.

(2.1) THEOREM. Let $L \triangleleft G$ and assume that every nonabelian simple group involved in $G / L$ and of order divisible by $p$ lies in the class $\mathcal{X}$. Let $P / L \in \operatorname{Syl}_{p}(G / L)$ and suppose that $\nu \in \operatorname{Irr}(L)$ is $P$-invariant. Then $\left|\operatorname{Irr}_{p^{\prime}}(G \mid \nu)\right|=\left|\operatorname{Irr}_{p^{\prime}}(N \mid \nu)\right|$, where $N=\mathbf{N}_{G}(P)$.

Proof. We proceed in a number of steps, working by induction on $|G / L|$.

Step 1. We can assume that $\nu$ is $G$-invariant.

Proof. Let $T=G_{\nu}$ be the stabilizer of $\nu$ in $G$. By hypothesis, $P \subseteq T$, and thus $|G: T|$ and $|N: N \cap T|$ are not divisible by $p$. By the Clifford correspondence, we know that induction defines bijections $\operatorname{Irr}(T \mid \nu) \rightarrow \operatorname{Irr}(G \mid \nu)$ and $\operatorname{Irr}((T \cap N) \mid \nu) \rightarrow \operatorname{Irr}(N \mid \nu)$, and since the indices of $T$ in $G$ and $T \cap N$ in $N$ are $p^{\prime}$-numbers, we see that $\left|\operatorname{Irr}_{p^{\prime}}(G \mid \nu)\right|=\left|\operatorname{Irr}_{p^{\prime}}(T \mid \nu)\right|$ and $\left|\operatorname{Irr}_{p^{\prime}}(N \mid \nu)\right|=\left|\operatorname{Irr}_{p^{\prime}}((N \cap T) \mid \nu)\right|$. If $T<G$, the inductive hypothesis guarantees that $\left|\operatorname{Irr}_{p^{\prime}}(T \mid \nu)\right|=\left|\operatorname{Irr}_{p^{\prime}}((T \cap N) \mid \nu)\right|$, and the result follows. We can thus assume that $T=G$, as claimed. 
Step 2. We can assume that $L \subseteq \mathbf{Z}(G)$, that $L$ is a $p^{\prime}$-group and that $\nu$ is faithful.

Proof. First, observe that if each character in $\operatorname{Irr}(P \mid \nu)$ has degree divisible by $p$, then the same is true about the sets $\operatorname{Irr}(G \mid \nu)$ and $\operatorname{Irr}(N \mid \nu)$, and thus $\left|\operatorname{Irr}_{p^{\prime}}(G \mid \nu)\right|=0=\left|\operatorname{Irr}_{p^{\prime}}(N \mid \nu)\right|$, and there is nothing further to prove. We can assume, therefore, that some member of $\operatorname{Irr}(P \mid \nu)$ has $p^{\prime}$-degree. In particular, $\nu(1)$ is not divisible by $p$.

By standard facts about character triples as in [9], the triple $(G, L, \nu)$ is isomorphic to a triple $\left(G^{*}, L^{*}, \nu^{*}\right)$, where $L^{*} \subseteq \mathbf{Z}\left(G^{*}\right)$ and $\nu^{*} \in \operatorname{Irr}\left(L^{*}\right)$ is faithful. In particular, $G / L \cong G^{*} / L^{*}$, and we let $P^{*} / L^{*}$ and $N^{*} / L^{*}$ be the subgroups of $G^{*} / L^{*}$ corresponding to $P / L$ and $N / L$ respectively. Then $P^{*} / L^{*}$ is a Sylow $p$-subgroup of $G^{*} / L^{*}$ and since $N / L=$ $\mathbf{N}_{G / L}(P / L)$, it follows that $N^{*} / L^{*}=\mathbf{N}_{G^{*} / L^{*}}\left(P^{*} / L^{*}\right)$, and we have $N^{*}=\mathbf{N}_{G^{*}}\left(P^{*}\right)$. The original hypotheses on $G, N, P$ and $L$, therefore, are reproduced with the corresponding subgroups in $G^{*}$.

By the definition of an isomorphism of character triples, we have bijections $\operatorname{Irr}(G \mid \nu) \rightarrow$ $\operatorname{Irr}\left(G^{*} \mid \nu^{*}\right)$ and $\operatorname{Irr}(N \mid \nu) \rightarrow \operatorname{Irr}\left(N^{*} \mid \nu^{*}\right)$. Also, we know that if $\theta$ and $\theta^{*}$ are characters that correspond under either of these bijections, then $\theta(1) / \nu(1)=\theta^{*}(1) / \nu^{*}(1)=\theta^{*}(1)$. Since $p$ does not divide $\nu(1)$, we conclude that $\left|\operatorname{Irr}_{p^{\prime}}(G \mid \nu)\right|=\left|\operatorname{Irr}_{p^{\prime}}\left(G^{*} \mid \nu^{*}\right)\right|$ and $\left|\operatorname{Irr}_{p^{\prime}}(N \mid \nu)\right|=$ $\left|\operatorname{Irr}_{p^{\prime}}\left(N^{*} \mid \nu^{*}\right)\right|$. It therefore suffices to prove the theorem with $G^{*}$ replacing $G$, and so we can assume that $L \subseteq \mathbf{Z}(G)$ and that the linear character $\nu \in \operatorname{Irr}(L)$ is faithful. It remains to be shown that we can assume that $|L|$ is a $p^{\prime}$-number.

We are assuming that there exists a character $\alpha \in \operatorname{Irr}(P \mid \nu)$ having $p^{\prime}$-degree. Since $|P / L|$ is a $p$-group and $L$ is abelian, $\alpha$ must have $p$-power degree, and thus $\alpha$ is linear and $\nu$ extends to $P$. Now write $\nu=\mu \lambda$, where $\mu$ has $p^{\prime}$-order and $\lambda$ has $p$-power order in the group of linear characters of $L$. Then $\lambda$ is a power of $\nu$, and hence $\lambda$ extends to $P$. But $P / L$ is a full Sylow $p$-subgroup of $G / L$ and $\lambda$ has $p$-power order, and thus $\lambda$ has an extension $\tau \in \operatorname{Irr}(G)$. (See, for example, Theorem 6.26 of [9].)

Multiplication by $\tau^{-1}$ defines a bijection $\operatorname{Irr}_{p^{\prime}}(G \mid \nu) \rightarrow \operatorname{Irr}_{p^{\prime}}(G \mid \mu)$ and similarly, multiplication by $\left(\tau_{N}\right)^{-1}$ defines a bijection $\operatorname{Irr}_{p^{\prime}}(N \mid \nu) \rightarrow \operatorname{Irr}_{p^{\prime}}(N \mid \mu)$. In this case, we see that it suffices to prove the theorem with $\mu$ in place of $\nu$, and with $L / \operatorname{ker}(\mu)$ in place of $L$. Since $|L / \operatorname{ker}(\mu)|=o(\mu)$ is a $p^{\prime}$-number, the proof of Step 2 is complete.

Step 3. We can assume that $G / L$ has a unique minimal normal subgroup $K / L$ and that $K P \triangleleft G$. Also, $L=\mathbf{Z}(G)$ and $K / L$ is nonabelian and has order divisible by $p$.

Proof. We can certainly assume that $L<G$, and we fix a minimal normal subgroup $K / L$ of $G / L$. Write $S=K P$ and $M=\mathbf{N}_{G}(S)$, and assume that $M<G$. Since $N \subseteq M$, the inductive hypothesis yields $\left|\operatorname{Irr}_{p^{\prime}}(M \mid \nu)\right|=\left|\operatorname{Irr}_{p^{\prime}}(N \mid \nu)\right|$, and so it suffices to prove that $\left|\operatorname{Irr}_{p^{\prime}}(G \mid \nu)\right|=\left|\operatorname{Irr}_{p^{\prime}}(M \mid \nu)\right|$.

Observe that $S$ permutes the set $\operatorname{Irr}_{p^{\prime}}(K \mid \nu)$, and that $M$ permutes the subset of this set consisting of the $S$-invariant members. Now let $\chi \in \operatorname{Irr}_{p^{\prime}}(G \mid \nu)$, and note that the irreducible constituents of $\chi_{K}$ lie in $\operatorname{Irr}_{p^{\prime}}(K \mid \nu)$, and similarly, if $\psi \in \operatorname{Irr}_{p^{\prime}}(M \mid \nu)$, the irreducible constituents of $\psi_{K}$ lie in $\operatorname{Irr}_{p^{\prime}}(K \mid \nu)$. We argue that both $\chi_{K}$ and $\psi_{K}$ have $S$-invariant irreducible constituents and that, in fact, the set of $S$-invariant irreducible constituents of each of these characters forms a single $M$-orbit. (Of course, the latter statement is clear for $\psi$ since $\psi \in \operatorname{Irr}(M)$, and so it requires proof only for $\chi$.)

Since $S / K$ is a $p$-group and $K$ acts trivially on $\operatorname{Irr}_{p^{\prime}}(K \mid \nu)$, it follows that all orbits in the action of $S$ on $\operatorname{Irr}_{p^{\prime}}(K \mid \nu)$ have $p$-power size. But the $S$-invariant characters $\chi_{K}$ and 
$\psi_{K}$ have $p^{\prime}$-degree, and so we see that each of them must have some $S$-invariant irreducible constituent. Suppose now that $\theta$ and $\theta^{\prime}$ are $S$-invariant constituents of $\chi_{K}$. Then $\theta^{\prime}=\theta^{g}$ for some element $g \in G$, and thus both $S$ and $S^{g}$ are contained in the stabilizer $T$ of $\theta^{\prime}$ in $G$. Since $S / K$ is a Sylow subgroup of $T / K$, it follows that $S^{g t}=S$ for some element $t \in T$. Then $g t \in M$ and $\theta^{\prime}=\theta^{g t}$, and thus $\theta^{\prime}$ and $\theta$ lie in the same $M$-orbit, as claimed.

Now let $\Delta$ be a set of representatives for the $M$-orbits of $S$-invariant characters in $\operatorname{Irr}_{p^{\prime}}(K \mid \nu)$. We have seen that each member of $\operatorname{Irr}_{p^{\prime}}(G \mid \nu)$ and of $\operatorname{Irr}_{p^{\prime}}(M \mid \nu)$ lies over a unique member of $\Delta$. It follows that

$$
\left|\operatorname{Irr}_{p^{\prime}}(G \mid \nu)\right|=\sum_{\delta \in \Delta}\left|\operatorname{Irr}_{p^{\prime}}(G \mid \delta)\right| \quad \text { and } \quad\left|\operatorname{Irr}_{p^{\prime}}(M \mid \nu)\right|=\sum_{\delta \in \Delta}\left|\operatorname{Irr}_{p^{\prime}}(M \mid \delta)\right|
$$

But $|G / K|<|G / L|$, and so we can apply the inductive hypothesis with $K, S$ and $\delta$ in place of $L, P$ and $\nu$ to conclude that $\left|\operatorname{Irr}_{p^{\prime}}(G \mid \delta)\right|=\left|\operatorname{Irr}_{p^{\prime}}(M \mid \delta)\right|$ for each character $\delta \in \Delta$. The result follows in this case, and so we can assume that $M=G$. Then $S \triangleleft G$, and in particular, $G / K$ is $p$-solvable.

Since we are done in the case that $G / L$ has a minimal normal subgroup $K / L$ such that $G / K$ fails to be $p$-solvable, we can assume that every factor group of $G / L$ by a minimal normal subgroup is $p$-solvable. On the other hand, if $G / L$ is $p$-solvable, we know that $\left|\operatorname{Irr}_{p^{\prime}}(G \mid \nu)\right|=\left|\operatorname{Irr}_{p^{\prime}}(N \mid \nu)\right|$ by Wolf's theorem [24]. We can thus assume that $G / L$ is not $p$-solvable, and hence it has a unique minimal normal subgroup $K / L$. Also, $K / L$ is not $p$-solvable, and so it is not abelian, and its order is divisible by $p$. Finally, $\mathbf{Z}(G)$ contains $L$ and is normal in $G$, but it does not contain $K$ since $K / L$ is nonabelian. Then $\mathbf{Z}(G)=L$, as required.

At this point, we know that $L=\mathbf{Z}(G)$ is a cyclic $p^{\prime}$-group with a faithful linear character $\nu$. Also, $K / L$ is a direct product of isomorphic simple groups in the class $\mathcal{X}$. Since $P / L \in \operatorname{Syl}_{p}(G / L)$, we see that we can write $P \cap K=L \times Q$, where $Q \in \operatorname{Syl}_{p}(K)$, and thus $N \subseteq \mathbf{N}_{G}(Q)$. By definition of the class $\mathcal{X}$, we know that there exists a subgroup $M$ and a character map ( )* satisfying conditions (a)-(c) of Theorem E.

Now $L \subseteq N \subseteq \mathbf{N}_{G}(Q) \subseteq M<G$, and so by the inductive hypothesis, we have $\left|\operatorname{Irr}_{p^{\prime}}(M \mid \nu)\right|=\left|\operatorname{Irr}_{p^{\prime}}(N \mid \nu)\right|$. Since $K \mathbf{N}_{G}(Q)=G$, we have $K M=G$, and we see that if we let $\Delta$ be a set of representatives for the $M$-orbits on $\operatorname{Irr}_{p^{\prime}}(K \mid \nu)$, then each member of $\operatorname{Irr}_{p^{\prime}}(G \mid \nu)$ lies over a unique member of $\Delta$. Also, since ( $)^{*}$ defines an $M$-permutation isomorphism, it follows that $\Delta^{*}=\left\{\theta^{*} \mid \theta \in \Delta\right\}$ is a set of representatives for the $M$-orbits on $\operatorname{Irr}_{p^{\prime}}((K \cap M) \mid \nu)$, and so each member of $\operatorname{Irr}_{p^{\prime}}(M \mid \nu)$ lies over a unique member of $\Delta^{*}$. Finally, we have

$$
\left|\operatorname{Irr}_{p^{\prime}}(G \mid \nu)\right|=\sum_{\theta \in \Delta}\left|\operatorname{Irr}_{p^{\prime}}(G \mid \theta)\right|=\sum_{\theta \in \Delta}\left|\operatorname{Irr}_{p^{\prime}}\left(N \mid \theta^{*}\right)\right|=\left|\operatorname{Irr}_{p^{\prime}}(M \mid \nu)\right|=\left|\operatorname{Irr}_{p^{\prime}}(N \mid \nu)\right|
$$

where the second equality follows by condition (c) of Theorem E. The result now follows. 


\section{Factor sets.}

We begin work now toward the definition of "good" simple groups and the proof of Theorem E. In this section we review some basic material about projective representations, factor sets and cohomology elements; we establish the notation that will be needed for the more technical material that follows, and we prove some preliminary results.

Let $K \triangleleft G$, and suppose that $\theta \in \operatorname{Irr}(K)$ is $G$-invariant. As is well known, $\theta$ determines a unique element in the Schur multiplier $M(G / K)=H^{2}\left(G / K, \mathbb{C}^{\times}\right)$, and we write $[\theta]_{G / K}$ to denote this cohomology element. The element $[\theta]_{G / K}$, together with information about the group $G / K$, determines the number of characters $\chi \in \operatorname{Irr}(G \mid \theta)$ for which the degree ratio $\chi(1) / \theta(1)$ is any given integer, and in particular if $\theta(1)$ is a $p^{\prime}$-number, then $[\theta]_{G / K}$ determines $\left|\operatorname{Irr}_{p^{\prime}}(G \mid \theta)\right|$.

The usual approach to defining the cohomology element $[\theta]_{G / K}$ is to start with a representation of $K$ affording $\theta$ and to extend it to an appropriate projective representation of $G$. This projective representation is associated with a certain factor set (i.e. 2-cocycle), which is a complex-valued function $f$ defined on $G \times G$ and satisfying $f(x, y) f(x y, z)=f(x, y z) f(y, z)$ for all elements $x, y, z \in G$. It is possible to choose the projective representation so that the factor set is constant on cosets of $K$ in each variable, and thus it defines a factor set of $G / K$. The corresponding element of $H^{2}\left(G / K, \mathbb{C}^{\times}\right)$is $[\theta]_{G / K}$. (See, for example, Chapter 11 of [9].)

For our purposes, it is more convenient to carry out the construction in the language of modules. But since the phrase "projective module" is taken, we will define a quasimodule to be the module analog of a projective representation. Specifically, if $G$ is any group and $V$ is a $\mathbb{C}$-space, we say that $V$ is a $G$-quasimodule if for each element $g \in G$, there is an invertible linear operator $v \mapsto v \bullet g$ defined on $V$ such that for $s, t \in G$, we have $(v \cdot s) \cdot t=f(s, t) v \bullet(s t)$ for some (nonzero) scalar $f(s, t)$. In this situation, it is easy to see that the function $f: G \times G \rightarrow \mathbb{C}$ is a factor set, and we say that the dot map is a quasiaction of $G$ on $V$.

Now let $K \triangleleft G$ and suppose that the dot action of $K$ on the $G$-quasimodule $V$ makes $V$ into a genuine $K$-module. (When this happens, we write $v k$ in place of $v \bullet k$ for $v \in V$ and $k \in K$.) Assume in addition that

(1) $(v k) \cdot g=v \cdot(k g)$ and

(2) $(v \cdot g) k=v \cdot(g k)$.

In this situation, we say that the $G$-quasimodule $V$ is well behaved with respect to the normal subgroup $K$, and we refer to it as a well behaved quasiextension of the $K$ module $V$. Note that if $f$ is the factor set associated with a $G$-quasimodule $V$, then $V$ is well behaved with respect to $K \triangleleft G$ if and only if $f(k, g)=1=f(g, k)$ for all $k \in K$ and $g \in G$. The significance of this is apparent in the following.

(3.1) LEMMA Let $K \triangleleft G$ and let $f$ be a factor set of $G$ such that $f(k, g)=1=f(g, k)$ for all $k \in K$ and $g \in G$. Then $f(k x, l y)=f(x, y)$ for all $k, l \in K$ and $x, y \in G$. In other words, $f$ is constant on cosets of $K$, and hence it defines a factor set on the group $G / K$.

Proof. It suffices to show that $f(k x, y)=f(x, y)$ and $f(x, y k)=f(x, y)$ for all $x, y \in G$ and $k \in K$. Since $f(k, x)=1=f(k, x y)$, we have

$$
f(k x, y)=f(k, x) f(k x, y)=f(k, x y) f(x, y)=f(x, y),
$$


as wanted. Similarly,

$$
f(x, y k)=f(x, y k) f(y, k)=f(x y, k) f(x, y)=f(x, y)
$$

and the proof is complete.

Now let $V$ be a quasimodule for $G$ that is well behaved with respect to $K \triangleleft G$. Let $g \in G$ and write $\sigma$ to denote the linear operator on $V$ defined by $v \mapsto v \bullet g$, for $v \in V$. Then

$$
v\left(g k g^{-1}\right) \sigma=\left(v\left(g k g^{-1}\right)\right) \cdot g=v \cdot\left(g k g^{-1} g\right)=v \cdot(g k)=(v \cdot g) k=(v \sigma) k,
$$

where the second and fourth equalities follow from the assumption that $V$ is well behaved with respect to $K$. This calculation motivates the following definition.

If $K \triangleleft G$ and $V$ is a $K$-module, we shall say that an invertible linear operator $\sigma: V \rightarrow V$ is compatible with an element $g$ in $G$ (with respect to $K$ ) if

$$
v\left(g k g^{-1}\right) \sigma=(v \sigma) k
$$

for all $k \in K$ and $v \in V$. Note that to test whether or not $\sigma: V \rightarrow V$ is compatible with $g$, it suffices to know the automorphism of $K$ induced by $g$; the actual element $g$ is irrelevant.

Our calculation shows that if $V$ is a $G$-quasimodule that is well behaved with respect to $K \triangleleft G$, then the dot-action of each element $g \in G$ on $V$ is compatible with $g$ with respect to $K$. To construct a well behaved quasiextension of a given $K$-module $V$, therefore, it is necessary that we find for each element $g \in G$, a linear operator on $V$ that is compatible with $g$ with respect to $K$.

(3.2) LEMMA. Let $V$ be a $K$-module, where $K \triangleleft G$, and let $\sigma, \tau: V \rightarrow V$.

(a) If $\sigma$ is compatible with $s \in G$, then $\sigma^{-1}$ is compatible with $s^{-1}$.

(b) If $\sigma$ and $\tau$ are compatible respectively with $s, t \in G$, then $\sigma \tau$ is compatible with st.

(c) If $V$ is irreducible as a $K$-module and $\sigma$ and $\tau$ are both compatible with $s \in G$, then $\tau=\alpha \sigma$ for some nonzero scalar $\alpha$.

Proof. If $\sigma$ is compatible with $s$, we have $v\left(s k s^{-1}\right) \sigma=v \sigma k$ for all $k \in K$ and $v \in V$. We can rewrite this by substituting $k$ for $s k s^{-1}$ and $v$ for $v \sigma$, and we obtain $\left(v \sigma^{-1}\right) k \sigma=$ $v\left(s^{-1} k s\right)$. Then $v\left(s^{-1} k s\right) \sigma^{-1}=v \sigma^{-1} k$, and so $\sigma^{-1}$ is compatible with $s^{-1}$, proving (a).

In situation (b), we have

$$
v\left(s t k t^{-1} s^{-1}\right) \sigma \tau=v \sigma\left(t k t^{-1}\right) \tau=v \sigma \tau k,
$$

where the first equality is valid since $\sigma$ is compatible with $s$ and the second holds because $\tau$ is compatible with $t$. This proves that $\sigma \tau$ is compatible with $s t$, as wanted.

Finally, for (c), we assume that $\sigma$ and $\tau$ are both compatible with $s$. Then $\tau^{-1}$ is compatible with $s^{-1}$ by (a), and we deduce from (b) that $\sigma \tau^{-1}$ is compatible with $1 \in G$. In other words, $\sigma \tau^{-1}$ commutes with the action of $K$ on the irreducible $K$-module $V$, and thus $\sigma \tau^{-1}$ is a scalar multiplication. 
Return now to the situation where $K \triangleleft G$ and $\theta \in \operatorname{Irr}(K)$ is $G$-invariant, and let $V$ be a $K$-module affording $\theta$. Our goal is to construct a well behaved quasiextension of the $K$-module $V$. In other words, we want a quasiaction of $G$ on $V$ that extends the $K$-action and that is well behaved with respect to $K$. (We shall consistently abuse language in this context and refer to $V$ as a quasiextension of the character $\theta$.)

Given $x \in G$, the maps $v \mapsto v\left(x k x^{-1}\right)$ for $k \in K$ make $V$ into a $K$-module affording the character $\theta^{x}=\theta$. There is thus an invertible linear operator $\sigma: V \rightarrow V$ that defines an isomorphism between these $K$-module structures on $V$. In other words, we have $v\left(x k x^{-1}\right) \sigma=v \sigma k$ for all $k \in K$ and $v \in V$, and thus $\sigma$ is compatible with $x$ on $V$. This shows that compatible operators exist for all elements $x \in G$.

For each element $x \in G$, fix a compatible operator $\sigma_{x}$ on $V$. Then $\sigma_{x y}$ is compatible with $x y$ for all $x, y \in G$, and by Lemma 3.2(b), so too is $\sigma_{x} \sigma_{y}$. By Lemma 3.2(c), it follows that $\sigma_{x} \sigma_{y}=f(x, y) \sigma_{x y}$ for some nonzero-scalar-valued function $f$. If we now define $v \cdot x=v \sigma_{x}$ for all $x \in G$, we see that this defines a quasiaction of $G$ on $V$. Our task now is to choose the linear operators $\sigma_{x}$ so that the resulting quasiaction will be well behaved with respect to $K$.

If $\sigma: V \rightarrow V$ is a linear operator and $k \in K$, write $k \sigma$ to denote the operator $v \mapsto(v k) \sigma$ and $\sigma k$ for the operator $v \mapsto(v \sigma) k$. Since the map $v \mapsto v k$ is clearly compatible with $k$, it follows from Lemma 3.2(b) that if $\sigma$ is compatible with $g \in G$, then $k \sigma$ is compatible with $k g$ and $\sigma k$ is compatible with $g k$.

Now fix a transversal $T$ for the cosets of $K$ in $G$. (Here and whenever we choose a coset transversal, we will always assume that it contains the identity element.) For each element $t \in T$, choose an invertible linear operator $\sigma_{t}$ on $V$ compatible with $t$, and do this so that $\sigma_{1}$ is the identity map. Now if $g \in G$, we can write $g=k t$, with $k \in K$ and $t \in T$. Because this decomposition is unique, we can define $v \bullet g=(v k) \sigma_{t}$.

(3.3) COROLLARY. The above construction defines a well behaved quasiextension of $\theta$ to $G$.

Proof. Since the linear operator $\sigma_{g}=k \sigma_{t}$ is compatible with $g=k t$ for all $g \in G$, we know that the map $v \mapsto v \bullet g=v \sigma_{g}$ makes $V$ into a $G$-quasimodule. Also, since $1 \in T$ and $\sigma_{1}$ is the identity, the quasiaction of $G$ on $V$ extends the original action of $K$.

What remains is to show that $(v l) \bullet g=v \bullet(l g)$ and $(v \bullet g) l=v \bullet(g l)$ for all $g \in G$ and $l \in K$. Writing $g=k t$, as before, we have $l g=l k t$, and thus

$$
v \cdot(l g)=v(l k) \sigma_{t}=(v l) \cdot(k t)=(v l) \cdot g
$$

as wanted. Finally, we have $g l=k t l=k\left(t l t^{-1}\right) t$, and thus

$$
v \cdot(g l)=v\left(k t l t^{-1}\right) \sigma_{t}=(v k)\left(t l t^{-1}\right) \sigma_{t}=(v k) \sigma_{t} l=(v \bullet g) l
$$

where the third equality holds because $\sigma_{t}$ is compatible with $t$. The proof is now complete.

It follows now that the original $K$-module $V$ affording the $G$-invariant character $\theta$ has a well behaved quasiextension to $G$, with a factor set $f$ defined on $G \times G$ and satisfying 
$f(g, k)=1=f(k, g)$ for all elements $k \in K$ and $g \in G$. Then $f$ is constant on cosets of $K$, and thus it defines a factor set of $G / K$. We define the cohomology element $[\theta]_{G / K}$ to be the cohomology class of $G / K$ determined by $f$, when $f$ is viewed as a factor set of $G / K$. The following shows that $[\theta]_{G / K}$ is well defined.

(3.4) LEMMA. All well behaved quasiextensions of $K$-modules affording the $G$-invariant character $\theta \in \operatorname{Irr}(K)$ yield the same cohomology element of $G / K$.

Proof. Let $V_{1}$ and $V_{2}$ be well behaved quasiextensions of $K$-modules affording $\theta$. Then $V_{1}$ and $V_{2}$ are isomorphic as $K$-modules, and so we can identify these spaces and assume that we have two quasiactions $v \bullet g$ and $v * g$ of $G$ on a single vector space $V$, where both quasiactions are well behaved with respect to $K$, and so in particular, $v \cdot k=v k=v * k$ for all $v \in V$ and $k \in K$.

We know that both maps $v \mapsto v \bullet g$ and $v \mapsto v * g$ are compatible with $g$. Since $V$ affords an irreducible character of $K$, it follows by Lemma 3.2(c) that $v * g=\alpha(g) v \bullet g$, for some scalar $\alpha(g)$, depending on $g$ but not on $v$. It is routine to check that the factor set $f$ associated with the dot-action and the factor set $f^{*}$ associated with the star-action are related by the formula

$$
f^{*}(x, y)=\frac{\alpha(x) \alpha(y)}{\alpha(x y)} f(x, y),
$$

and thus $f$ and $f^{*}$ are cohomologous as factor sets of $G$. To complete the proof, we must show that $f$ and $f^{*}$ are cohomologous when they are viewed as factor sets of $G / K$, and for this purpose, it suffices to show that $\alpha$ is constant on cosets of $K$.

Using the fact that both quasiactions are well behaved with respect to $K$, we have

$$
\alpha(k g) v \cdot(k g)=v *(k g)=(v k) * g=\alpha(g)(v k) \cdot g=\alpha(g) v \cdot(k g) .
$$

Thus $\alpha(k g)=\alpha(g)$ and $\alpha$ is constant on cosets of $K$, as required.

Next, we present an observation that will allow us to compute the factor set $f$ associated with a well behaved quasiextension of a $K$-module, where $K \triangleleft G$. Note that since $f$ is constant on cosets of $K$, it suffices to compute $f(s, t)$, where $s$ and $t$ run over a transversal for the cosets of $K$ in $G$.

(3.5) LEMMA Let $K \triangleleft G$ and let $V$ be a $G$-quasimodule that is well behaved with respect to $K$. Fix a transversal $T$ for $K$ in $G$ and let $\sigma_{t}: V \rightarrow V$ be the linear operator $v \mapsto v \bullet t$ for $t \in T$. Now given $s, t \in T$, write $s t=k u$, where $k \in K$ and $u \in T$ depend on $s$ and $t$. Then

$$
f(s, t) v k \sigma_{u}=v \sigma_{s} \sigma_{t}
$$

for all $v \in V$.

Proof. By the definition of $f$, we know that

$$
f(s, t) v \cdot(s t)=(v \cdot s) \cdot t=v \sigma_{s} \sigma_{t} .
$$

and the result follows because $v \cdot(s t)=v \bullet(k u)=(v k) \bullet u=v k \sigma_{u}$. 
Let $K \triangleleft G$, as before. Suppose that $N \subseteq G$ with $K N=G$, and let $H=K \cap N$. Let $\theta \in \operatorname{Irr}(K)$ and $\theta^{\prime} \in \operatorname{Irr}(H)$ be stabilized by $N$. (Note that $\theta$ is $N$-invariant if and only if it is $G$-invariant.) In this situation, we have cohomology elements $[\theta]_{G / K}$ and $\left[\theta^{\prime}\right]_{N / H}$, which, of course, lie in different cohomology groups. But $G / K$ and $N / H$ are naturally isomorphic, and so we can identify their Schur multipliers via the natural map. It is therefore meaningful to compare $[\theta]_{G / K}$ and $\left[\theta^{\prime}\right]_{N / H}$, and in particular, we can ask if these cohomology elements are equal. (Finding conditions where such equality holds is the main goal of the next several sections of this paper.) Note that if equality does hold, then there is a bijection from $\operatorname{Irr}(G \mid \theta)$ to $\operatorname{Irr}\left(N \mid \theta^{\prime}\right)$ such that if $\chi$ and $\chi^{\prime}$ correspond, we have $\chi(1) / \theta(1)=\chi^{\prime}(1) / \theta^{\prime}(1)$.

An easy example where we have equality of cohomology elements is the following, which we will use later.

(3.6) LEMMA. Let $K \triangleleft G$ and suppose $N \subseteq G$ and $K N=G$. Let $\theta \in \operatorname{Irr}(K)$ be $G$-invariant and suppose that $\theta_{H}=\theta^{\prime}$ is irreducible, where $H=K \cap N$. Then $[\theta]_{G / K}=$ $\left[\theta^{\prime}\right]_{N / H}$.

Proof. Let $V$ be a $K$-module affording $\theta$ and choose a well behaved quasiextension to $G$, with factor set $f$. The restriction of the quasiaction of $G$ to $N$ yields a well behaved quasiextension to $N$ of $V$, where now we view $V$ as an $H$-module that affords $\theta^{\prime}$. The restriction of $f$ to $N$ is a factor set $f^{\prime}$ of $N$ that is constant on cosets of $H$ in $N$. Now view $f$ as a factor set of $G / K$ and $f^{\prime}$ as a factor set of $N / H$.

The natural isomorphism from $N / H$ to $G / K$ maps a coset of $H$ in $N$ to the coset of $K$ in $G$ that contains it. It follows that under this identification, we have $f=f^{\prime}$, and thus $[\theta]_{G / K}=\left[\theta^{\prime}\right]_{N / H}$ as wanted.

Suppose that $V$ is a well behaved quasiextension to $G$ of $\theta \in \operatorname{Irr}(K)$, where $K \triangleleft G$ and $\theta$ is $G$-invariant. Let $C \subseteq \mathbf{C}_{G}(K)$ and suppose $c \in C$. Since the linear operator $v \mapsto v \bullet c$ on $V$ is compatible with $c$ and $c$ centralizes $K$, we see that this operator commutes with the action of $K$ on $V$. Because $V$ is irreducible as a $K$-module, it follows that $v \bullet c=\rho(c) v$ for some scalar $\rho(c)$ depending on $c$ (but not on $v$ ).

We define the $C$-type of the quasimodule $V$ to be an ordered pair consisting of two complex-valued functions. The first of these is the function $\rho: C \rightarrow \mathbb{C}$ that we have just defined. The second component of the $C$-type of $V$ is the function of two variables defined on $(C K / K) \times(G / K)$ and obtained by viewing the factor set $f$ associated with $V$ as being defined on $(G / K) \times(G / K)$ and then restricting the first variable to the subgroup $C K / K$. The $C$-type of a quasiextension of $\theta$ will play an important role in what follows.

The following technical lemma will be useful.

(3.7) LEMMA. Let $K \triangleleft G$ and $C \triangleleft G$ with $[K, C]=1$, and let $Z=K \cap C$, so that $Z \subseteq \mathbf{Z}(K)$. Let $N \subseteq G$ such that $K N=G$ and $C \subseteq N$, and write $H=K \cap N$. Let $\nu \in \operatorname{Irr}(Z)$ and suppose that $\theta \in \operatorname{Irr}(K)$ and $\theta^{\prime} \in \operatorname{Irr}(H)$ both lie over $\nu$. Assume that both $\theta$ and $\theta^{\prime}$ are $N$-invariant, so that $\theta$ is actually $G$-invariant. Then there exist well behaved quasiextensions $V$ and $V^{\prime}$ of $\theta$ and $\theta^{\prime}$ to $G$ and $N$ such that $V$ and $V^{\prime}$ have identical C-types.

Of course, for this to make sense, we must consider the factor set $f$ for $V$ as defined 
on $G / K$ and the factor set $f^{\prime}$ for $V^{\prime}$ as being defined on $N / H$, and then we must identify $G / K=N / H$ via the natural isomorphism.

Proof of Lemma 3.7. Let $V$ and $V^{\prime}$ be modules affording $\theta$ and $\theta^{\prime}$ on $K$ and $H$ respectively. Choose a transversal $R$ for $Z=H \cap C$ in $C$ and a transversal $S$ for $H C$ in $N$. Then the set $T=R S$ is a transversal for $H$ in $N$ and also for $K$ in $G$. It suffices to construct quasiextensions of $V$ and $V^{\prime}$ with factor sets $f$ and $f^{\prime}$ such that $f(r, q s)=f^{\prime}(r, q s)$ for all elements $r, q \in R$ and $s \in S$ and to check that each element $c \in C$ acts via the same scalar on both $V$ and $V^{\prime}$

To construct our quasiextensions, we choose for each element $t \in T$, a linear operator $\sigma_{t}$ on $V$ and a linear operator $\sigma_{t}^{\prime}$ on $V^{\prime}$ such that $\sigma_{t}$ and $\sigma_{t}^{\prime}$ are compatible with $t$ with respect to $K$ and $H$, respectively. Since the elements of $R$ centralize $K$ and $H$, we see that every linear operator on $V$ or $V^{\prime}$ that is compatible with $s \in S$ will also be compatible with $r s$ for all $r \in R$. We can thus choose our linear operators such that $\sigma_{r s}=\sigma_{s}$ for all elements $r \in R$ and $s \in S$, and similarly for the operators $\sigma^{\prime}$ on $V^{\prime}$. (Recall that we are assuming that $1 \in R$ so that $S \subseteq T$.) Also, we assume that $1 \in S$ and that $\sigma_{1}$ and $\sigma_{1}^{\prime}$ are identity maps, and thus $\sigma_{r}$ and $\sigma_{r}^{\prime}$ are identity maps for all $r \in R$. These choices determine well behaved quasiextensions of $\theta$ and $\theta^{\prime}$ and we let $f$ and $f^{\prime}$ be the corresponding factor sets of $G$ and of $N$.

Now let $r, q \in R$. We can then write $r q=z p$ for elements $z \in Z$ and $p \in R$. If $s \in S$, we have $r(q s)=z(p s)$, and hence by Lemma 3.5, we have

$$
f(r, q s) v z \sigma_{p s}=v \sigma_{r} \sigma_{q s} .
$$

But $\sigma_{r}$ is the identity and $\sigma_{p s}=\sigma_{s}=\sigma_{q s}$. Also, $z$ acts on $V$ via the scalar $\nu(z)$. It follows that $f(r, q s) \nu(z) v=v$, and thus $f(r, q s)=\nu(z)^{-1}$. A similar calculation shows that $f^{\prime}(r, q s)=\nu(z)^{-1}$, and so $f(r, q s)=f^{\prime}(r, q s)$, as wanted.

Now if $c \in C$ is arbitrary, we can write $c=z r$ for some element $z \in Z$ and $r \in R$. Then for $v \in V$, we have

$$
v \cdot c=v \cdot z r=(v z) \cdot r=\nu(z) v \sigma_{r}=\nu(z) v
$$

and thus $\rho(c)=\nu(z)$ for the quasimodule $V$. A similar calculation yields the same result for $V^{\prime}$ and the proof is complete.

\section{Factor set induction.}

Let $G_{0} \subseteq G$, where $G$ is an arbitrary finite group, and fix a transversal $T$ for the right cosets of $G_{0}$ in $G$. The action of $G$ on the right cosets of $G_{0}$ induces an action of $G$ on $T$, and we indicate this action with a dot. If $t \in T$ and $x \in G$, therefore, then $t \cdot x$ is the unique element of $T$ in the coset $\left(G_{0}\right) t x$. Of course, this is really an action, and so we have $(t \cdot x) \cdot y=t \cdot x y$ for all elements $t \in T$ and $x, y \in G$. Note also that $t x(t \cdot x)^{-1}$ is in $G_{0}$ for all $t \in T$ and $x \in G$.

Now suppose that $f$ is a factor set on $G_{0}$. We define the induced function $f^{G}$ on $G \times G$ by setting

$$
f^{G}(x, y)=\prod_{t \in T} f\left(t x(t \cdot x)^{-1},(t \cdot x) y(t \cdot x y)^{-1}\right)
$$


We will see that $f^{G}$ is a factor set on $G$, and that the map $f \mapsto f^{G}$ defines a map on cohomology groups. Since this is really the standard corestriction map, we should, perhaps, refer to $f^{G}$ as the "corestricted" function associated with $f$. We will see, however, that there is a connection between $f^{G}$ and tensor induction of modules, and so from our view, it seems more natural to refer to $f^{G}$ as the "induced" function.

We show now that $f^{G}$ is a factor set of $G$. To see this, let $x, y, z \in G$ and write $u_{t}=$ $t x(t \cdot x)^{-1}, v_{t}=(t \cdot x) y(t \cdot x y)^{-1}$ and $w_{t}=(t \cdot x y) z(t \cdot x y z)^{-1}$. Note that $u_{t} v_{t}=t x y(t \cdot x y)^{-1}$ and that $v_{t} w_{t}=(t \cdot x) y z(t \cdot x y z)^{-1}$. We compute

$$
\begin{aligned}
f^{G}(x, y) f^{G}(x y, z) & =\prod_{t \in T} f\left(u_{t}, v_{t}\right) f\left(u_{t} v_{t}, w_{t}\right) \\
& =\prod_{t \in T} f\left(u_{t}, v_{t} w_{t}\right) f\left(v_{t}, w_{t}\right) \\
& =f^{G}(x, y z) f^{G}(y, z),
\end{aligned}
$$

and it follows that $f^{G}$ is a factor set on $G$, as claimed.

Suppose now that $K \triangleleft G$ with $K \subseteq G_{0}$, and assume that $f$ is constant on cosets of $K$ in $G_{0}$. It follows easily that $f^{G}$ is constant on cosets of $K$ in $G$. In this situation, if we view $f$ as a factor set on $G_{0} / K$ and we induce to $G / K$ using the image of the transversal $T$ modulo $K$, the resulting factor set of $G / K$ is just the factor set $f^{G}$ viewed as a factor set of $G / K$. In other words, when $f^{G}$ is viewed as a factor set of $G / K$, it is equal to $f^{G / K}$.

Now if $g$ is another factor set of $G_{0}$ that is cohomologous with $f$, we claim that $f^{G}$ and $g^{G}$ are cohomologous as factor sets of $G$. To see this, write $f=g h$, where $h(u, v)=a(u) a(v) a(u v)^{-1}$ and $a$ is some scalar-valued function on $G_{0}$. Clearly, then $f^{G}=g^{G} h^{G}$. To compute $h^{G}$, we define

$$
b(x)=\prod_{t \in T} a\left(t x(t \cdot x)^{-1}\right)
$$

for elements $x \in G$. We then have

$$
\begin{aligned}
h^{G}(x, y) & =\prod_{t \in T} h\left(t x(t \cdot x)^{-1},(t \cdot x) y(t \cdot x y)^{-1}\right) \\
& =\prod_{t \in T} a\left(t x(t \cdot x)^{-1}\right) a\left((t \cdot x) y(t \cdot x y)^{-1}\right) a\left(t x y(t \cdot x y)^{-1}\right)^{-1} \\
& =b(x) b(y) b(x y)^{-1} .
\end{aligned}
$$

This shows that $h^{G}$ is cohomologously trivial, and thus $f^{G}$ and $g^{G}$ are cohomologous, as claimed.

We mention also that if $f$ is a factor set of $G_{0}$ and we compute $f^{G}$ using two different transversals for the right cosets of $G_{0}$ in $G$, then the resulting induced factor sets are cohomologous. But since we will not have occasion to vary our choice of transversal, we will not actually need this observation.

Next, we generalize the process of tensor induction of modules to quasimodules. Suppose that $K \triangleleft G$ with $K \subseteq G_{0}$, and let $V$ be a $G_{0}$-quasimodule that is well behaved with 
respect to $K$ and has factor set $f$. Assign an arbitrary but fixed order to the members of the right transversal $T$ of $G_{0}$ in $G$. We can then write $T=\left\{t_{1}, t_{2}, \ldots, t_{e}\right\}$, where $e=|T|=\left|G: G_{0}\right|$. Write $V^{\otimes G}=V \otimes V \otimes \cdots \otimes V$, where there are $e$ factors. If a vector $v_{t} \in V$ is selected for each element $t \in T$, we shall write $\bigotimes_{t \in T} v_{t}$ to denote the vector $v_{t_{1}} \otimes v_{t_{2}} \otimes \cdots \otimes v_{t_{e}}$

Now we make $V^{\otimes G}$ into a $G$-quasimodule by setting

$$
\left(\bigotimes_{t \in T} v_{t}\right) \cdot g=\bigotimes_{t \in T} w_{t}
$$

where $w_{t} \in V$ is defined for all $t \in T$ by the formula

$$
w_{t} \cdot g=\left(v_{t}\right) \cdot\left(t g(t \cdot g)^{-1}\right) .
$$

(Note that for each element $g \in G$, this uniquely determines an invertible linear operator on $V^{\otimes G}$. We will show that what results is a quasiaction of $G$ on $V^{\otimes G}$.) In the following computation we use dots to denote three different things: the given quasiaction of $G_{0}$ on $V$, the quasiaction of $G$ on $V^{\otimes G}$ that we have just defined and the action of $G$ on the transversal $T$, as before. We trust that this will not cause too much confusion.

If $x, y \in G$, and vectors $u_{t} \in V$ are arbitrary, we compute $\left(\left(\bigotimes u_{t}\right) \cdot x\right) \bullet y$. Write $\left(\otimes u_{t}\right) \cdot x=\bigotimes v_{t}$ and $\left(\otimes v_{t}\right) \cdot y=\bigotimes w_{t}$. We then have

$$
v_{t} \cdot x=\left(u_{t}\right) \cdot\left(t x(t \cdot x)^{-1}\right)
$$

and

$$
w_{t} \cdot y=\left(v_{t}\right) \cdot\left(t y(t \cdot y)^{-1}\right) .
$$

Substitute $t \cdot x$ for $t$ in the latter equation to get

$$
\begin{aligned}
w_{t} \cdot x y & =\left(v_{t} \cdot x\right) \cdot\left((t \cdot x) y(t \cdot x y)^{-1}\right) \\
& =\left(\left(u_{t}\right) \cdot\left(t x(t \cdot x)^{-1}\right)\right) \cdot\left((t \cdot x) y(t \cdot x y)^{-1}\right) \\
& =f\left(t x(t \cdot x)^{-1},(t \cdot x) y(t \cdot x y)^{-1}\right) u_{t} \cdot\left(t x y(t \cdot x y)^{-1}\right) .
\end{aligned}
$$

On the other hand, the $(t \cdot x y)$-factor of $\left(\bigotimes u_{t}\right) \cdot(x y)$ is $u_{t} \cdot\left(t x y(t \cdot x y)^{-1}\right)$. It follows that

$$
\left(\left(\bigotimes u_{t}\right) \cdot x\right) \cdot y=\prod_{t \in T} f\left(t x(t \cdot x)^{-1},(t \cdot x) y(t \cdot x y)^{-1}\right)\left(\bigotimes u_{t}\right) \cdot(x y)
$$

We see now that this construction makes $V^{\otimes G}$ into a $G$-quasimodule with factor set $f^{G}$. We refer to this as the tensor-induced quasimodule.

Recall now that we have $K \triangleleft G$ with $K \subseteq G_{0}$ and that the $G_{0}$-quasimodule $V$ is well behaved with respect to $K$. If $k \in K$, then $t \cdot k=t$, and hence $t k(t \cdot k)^{-1}=t k t^{-1} \in K$. It follows that $f^{G}(k, x)=1$ for all $x \in G$, and similarly, $f^{G}(x, k)=1$ for all $x \in G$. We 
conclude that our $G$-quasimodule $V^{\otimes G}$ is well behaved with respect to $K$. In particular, $V^{\otimes G}$ is a $K$-module. Also, we see that if $k \in K$ then

$$
\left(\bigotimes u_{t}\right) k=\bigotimes u_{t}\left(k t^{-1}\right)
$$

and it follows that the character $\theta$ of $K$ afforded by $V^{\otimes G}$ is exactly $\prod_{t \in T} \beta^{t}$, where $\beta$ is the character of $K$ afforded by $V$.

Finally, suppose that $\beta$ and $\theta$ are irreducible characters of $K$, and let $C \triangleleft G$ with $C \subseteq \mathbf{C}_{G_{0}}(K)$. Recall that the $C$-type of $V$ consists of two functions. One of these is simply the factor set $f$ (viewed on $G_{0} / K$ ) with the first variable restricted to elements of $K C / K$. The other component of the $C$-type is the scalar-valued function $\rho$ on $C$ defined by the formula $v \bullet c=\rho(c) v$. Now to compute the $C$-type of $f^{G}$, we let $c \in C$ and $g \in G$ and we compute

$$
f^{G}(c, g)=\prod_{t \in T} f\left(t c(t \cdot c)^{-1},(t \cdot c) g(t \cdot c g)^{-1}\right)
$$

But $C \triangleleft G$ and $C \subseteq G_{0}$, and it follows that $t \cdot c=t$ for all $t \in T$. Also $t c t^{-1} \in C$, and so each factor in the above product is known if we know the $C$-type of $V$. Also, since $t \cdot c=t$ for $t \in T$ and $c \in C$, we see that

$$
\left(\bigotimes v_{t}\right) \cdot c=\bigotimes\left(v_{t} \cdot\left(t c t^{-1}\right)\right)
$$

and so $c$ acts via the scalar $\prod_{t \in T} \rho\left(t c t^{-1}\right)$. We have proved the following.

(4.1) THEOREM. Let $K \triangleleft G$ and suppose that $\beta \in \operatorname{Irr}(K)$ has stabilizer $G_{0}$ in $G$ and that the product of the $\left|G: G_{0}\right|$ distinct $G$-conjugates of $\beta$ is a character $\theta \in \operatorname{Irr}(K)$. Let $V$ be a well behaved quasiextension to $G_{0}$ of a $K$-module affording $\beta$, and let $f$ be the corresponding factor set. Fix a transversal for the right cosets of $G_{0}$ in $G$. Then $V^{\otimes G}$ is a well behaved quasiextension of a $K$-module affording $\theta$, and $f^{G}$ is the corresponding factor set. Also if $C \triangleleft G$ centralizes $K$, then the $C$-type of $V^{\otimes G}$ is determined by the $C$-type of $V$ and the choice of transversal.

We stress that we have computed a specific factor set corresponding to the tensorinduced quasimodule, and not just its cohomology class. This will be significant in what follows.

\section{Central Products.}

In this short section, we review some known material. Suppose that $K / \mathbf{Z}(K)$ is a direct product of nonabelian simple subgroups. (We saw this situation in Section 1, but there, the simple factors were all isomorphic, which we are not assuming now.) Write $Z=\mathbf{Z}(K)$ and $K / Z=\prod U_{i} / Z$, where the product is direct and $U_{i} / Z$ is simple for all subscripts $i$. If $i \neq j$, we have $\left[U_{i}, U_{j}\right] \subseteq U_{i} \cap U_{j}=Z$, and thus $\left[U_{i}, U_{j}, U_{j}\right]=1$. It follows by the three-subgroups lemma that $U_{i}$ centralizes $\left(U_{j}\right)^{\prime}$. Because $U_{j} / Z$ is nonabelian and simple, however, we have $U_{j}=\left(U_{j}\right)^{\prime} Z$, and thus $U_{i}$ centralizes $U_{j}$.

Now consider a more general situation. Suppose that a group $K$ is a product of subgroups $U_{i}$, and let $Z=\bigcap U_{i}$. We say that $K$ is the central product of the $U_{i}$ 
provided that $K / Z$ is the direct product of its subgroups $U_{i} / Z$ and $\left[U_{i}, U_{j}\right]=1$ for $i \neq j$. It is clear in this situation that $Z \subseteq \mathbf{Z}(K)$ and that $U_{i} \triangleleft K$ for all $i$.

An important special case is where a group $K$ factors as a product of just two subgroups. If $K=A B$, where $[A, B]=1$, then $K$ is automatically the central product of $A$ and $B$.

We need to consider the irreducible characters of central products. The basic facts are summarized in the following (known) result.

(5.1) LEMMA. Let $K=\prod_{i=1}^{e} U_{i}$ be a central product with $Z=\bigcap U_{i}$, and let $\nu$ be a linear character of $Z$. Given characters $\theta_{i} \in \operatorname{Irr}\left(U_{i} \mid \nu\right)$, there exists a unique character $\chi \in \operatorname{Irr}(K \mid \nu)$ such that $\chi_{U_{i}}$ is a multiple of $\theta_{i}$ for all $i$. Also, if $u_{i} \in U_{i}$, then

$$
\chi\left(u_{1} u_{2} \cdots u_{e}\right)=\theta_{1}\left(u_{1}\right) \theta_{2}\left(u_{2}\right) \cdots \theta_{e}\left(u_{e}\right) .
$$

Furthermore, every character $\chi \in \operatorname{Irr}(K)$ arises in this way.

Proof. Let $K^{*}$ be the external direct product of the groups $U_{i}$ and observe that there is a natural surjection from $K^{*}$ to $K$ carrying the $e$-tuple $\left(u_{1}, \ldots, u_{e}\right)$ to the product $u_{1} \cdots u_{e}$. Every character $\chi \in \operatorname{Irr}(K)$, therefore, can be lifted to a unique character $\chi^{*} \in \operatorname{Irr}\left(K^{*}\right)$. But $K^{*}$ is a direct product, and so we can write $\chi^{*}=\theta_{1} \times \cdots \times \theta_{e}$, where $\theta_{i} \in \operatorname{Irr}\left(U_{i}\right)$. We thus have

$$
\chi\left(u_{1} \cdots u_{e}\right)=\chi^{*}\left(\left(u_{1}, \ldots, u_{e}\right)\right)=\theta_{1}\left(u_{1}\right) \cdots \theta_{e}\left(u_{e}\right) .
$$

In particular, $\chi(1)=\prod \theta_{i}(1)$ and we see that $\chi_{U_{i}}$ is a multiple of $\theta_{i}$.

As $Z \subseteq \mathbf{Z}(K)$, we know that $\chi \in \operatorname{Irr}(K \mid \nu)$ for some linear character $\nu$ of $Z$, and thus $\chi(z)=\chi(1) \nu(z)$ for all $z \in Z$. In the formula of the previous paragraph, we can choose $i$ arbitrarily and set $u_{i}=z$ and $u_{j}=1$ for $j \neq i$. This yields

$$
\chi(1) \nu(z)=\chi(z)=\theta_{i}(z) \prod_{j \neq i} \theta_{j}(1)=\frac{\chi(1)}{\theta_{i}(1)} \theta_{i}(z),
$$

and thus $\theta_{i}(z)=\theta_{i}(1) \nu(z)$. Thus $\theta_{i} \in \operatorname{Irr}\left(U_{i} \mid \nu\right)$, as wanted.

To complete the proof, we must show that if we choose $\theta_{i} \in \operatorname{Irr}\left(U_{i} \mid \nu\right)$ arbitrarily, then the character $\chi^{*}=\theta_{1} \times \cdots \times \theta_{e}$ in $\operatorname{Irr}\left(K^{*}\right)$ is the lift of some character of $K$. We must show, in other words, that if $\left(u_{1}, \ldots, u_{e}\right)$ is in the kernel of our surjection $K^{*} \rightarrow K$, then this element lies in $\operatorname{ker}\left(\chi^{*}\right)$. We have $u_{1} \cdots u_{e}=1$, and since $\prod U_{i} / Z$ is direct, it follows that $u_{i} \in Z$ for all $i$, and thus $\theta_{i}\left(u_{i}\right)=\theta_{i}(1) \nu\left(u_{i}\right)$. We have

$$
\chi^{*}\left(\left(u_{1}, \ldots, u_{e}\right)\right)=\prod \theta_{i}\left(u_{i}\right)=\prod \theta_{i}(1) \prod \nu\left(u_{i}\right)=\chi(1) \nu\left(\prod u_{i}\right)=\chi(1)
$$

and we are done.

In the situation of Lemma 5.1, we refer to $\chi$ as the dot product of the characters $\theta_{i}$ and we write $\chi=\theta_{1} \cdot \cdots \cdot \theta_{e}$.

Although we shall need this generality later, we specialize now to the case where there are just two factors. Let $K=A B$ be a central product, and write $Z=A \cap B$. Let 
$\nu \in \operatorname{Irr}(Z)$ and suppose that $\alpha \in \operatorname{Irr}(A)$ and $\beta \in \operatorname{Irr}(B)$ lie over $\nu$. Given a module $V$ affording $\alpha$ and a module $W$ affording $\beta$, there is a natural way to make $V \otimes W$ into a $K$-module affording $\alpha \cdot \beta$. We define $(v \otimes w)(a b)=v a \otimes w b$ for $a \in A$ and $b \in B$. (In other words, the linear operator on $V \otimes W$ induced by $a b$ is $\sigma \otimes \tau$, where $\sigma$ and $\tau$ are the linear operators on $V$ and $W$ induced by $a$ and $b$.) To see that this is well defined, suppose that $a b=a^{\prime} b^{\prime}$ with $a^{\prime} \in A$ and $b^{\prime} \in B$. Then $a^{-1} a^{\prime}=b\left(b^{\prime}\right)^{-1}$ lies in $Z$, and so if we write $z$ to denote this element, we know that $z$ acts like the scalar $\nu(z)$ on both $V$ and $W$. It follows for $v \in V$ and $w \in W$ that $v a^{\prime}=\nu(z) v a$ and $w b^{\prime}=\nu(z)^{-1} w b$, and thus $v a^{\prime} \otimes w b^{\prime}=v a \otimes w b$, as required.

\section{Central products and factor sets.}

In this section, we suppose that $K \triangleleft G$ and $C \triangleleft G$ with $[K, C]=1$, and we write $K \cap C=Z$. Let $\theta \in \operatorname{Irr}(K)$ and $\gamma \in \operatorname{Irr}(C)$ be $G$-invariant and lie over a common linear character $\nu$ of $Z$. In this case, $K C$ is a central product and the character $\theta \bullet \gamma$ is defined and is $G$-invariant. We study the cohomology element $[\theta \bullet \gamma]_{G / K C}$.

Recall that if $V$ and $W$ afford $\theta$ and $\gamma$, then $V \otimes W$ affords $\theta \cdot \gamma$ with the (well defined) action $(v \otimes w)(k c)=v k \otimes w c$ for $k \in K$ and $c \in C$. We construct a particular well behaved quasiextension of $V \otimes W$ to $G$, and we compute the corresponding factor set $f$ of $G / K C$.

(6.1) LEMMA. Assume the above notation and let $t \in G$. If $\sigma: V \rightarrow V$ and $\tau: W \rightarrow W$ are linear operators compatible with $t$, then $\sigma \otimes \tau$ is compatible with $t$ on $V \otimes W$.

Proof. We have

$$
\begin{aligned}
(v \otimes w)\left(t(k c) t^{-1}\right)(\sigma \otimes \tau) & =(v \otimes w)\left(t k t^{-1}\right)\left(t c t^{-1}\right)(\sigma \otimes \tau) \\
& =v\left(t k t^{-1}\right) \sigma \otimes w\left(t c t^{-1}\right) \tau \\
& =v \sigma k \otimes w \tau c \\
& =(v \otimes w)(\sigma \otimes \tau)(k c)
\end{aligned}
$$

as required, where $v \in V, w \in W, k \in K$ and $c \in C$ are arbitrary.

We need the following elementary lemma about tensor products over fields.

(6.2) LEMMA. Let $V$ and $W$ be nonzero vector spaces. Let $\mu$ and $\mu^{\prime}$ be linear operators on $V$ and let $\nu$ and $\nu^{\prime}$ be linear operators on $W$, where $\mu^{\prime}$ and $\nu^{\prime}$ are invertible. Suppose that $\mu \otimes \nu=\mu^{\prime} \otimes \nu^{\prime}$ as linear operators on $V \otimes W$. Then there exists a nonzero scalar $\alpha$ such that $\mu=\alpha \mu^{\prime}$ and $\nu=(1 / \alpha) \nu^{\prime}$.

Proof. It is no loss to assume that $\mu^{\prime}$ and $\nu^{\prime}$ are the identity operators on $V$ and $W$. Then $v \mu \otimes w \nu=v \otimes w$ for all $v \in V$ and $w \in W$.

Suppose that $v$ and $v \mu$ are linearly independent for some vector $v \in V$. Then the equation $v \otimes w=v \mu \otimes w \nu$ forces $w=0$. Since $W \neq 0$, we deduce that $\mu$ maps every onedimensional subspace of $V$ into itself. But $\mu$ is linear, and this forces $\mu$ to be multiplication by some scalar $\alpha$. Also, $\alpha \neq 0$ since there exist $v$ and $w$ with $v \otimes w$ nonzero. The result now follows.

Now let $K \triangleleft G$ and $C \triangleleft G$ be as before, with $[K, C]=1$, and $Z=K \cap C$. Also as before, let $\theta \in \operatorname{Irr}(K)$ and $\gamma \in \operatorname{Irr}(C)$ be $G$-invariant and lie over the linear character $\nu$ of $Z$, and let $V$ and $W$ afford $\theta$ and $\gamma$, respectively, so that $V \otimes W$ affords $\theta \bullet \gamma$ on $K C$. 
To compute $[\theta \cdot \gamma]_{G / K C}$, we build a well behaved quasiextension of $V \otimes W$ to $G$ as in Section 3. We choose a transversal $T$ for the cosets of $K C$ in $G$ (with $1 \in T$ ) and for each element $t \in T$, we construct an operator on $V \otimes W$ that is compatible with $t$. To do this, we choose (and hold fixed) linear operators $\sigma_{t}$ on $V$ and $\tau_{t}$ on $W$, each of them compatible with $t$. (And we take $\sigma_{1}$ and $\tau_{1}$ to be identity operators.) By Lemma 6.1, the operator $\sigma_{t} \otimes \tau_{t}$ is compatible with $t$ on the $K C$-module $V \otimes W$, and we use these operators to determine our quasiextension.

To compute the corresponding factor set $f$, which will be constant on cosets of $K C$, it suffices to determine $f(s, t)$ for $s, t \in T$.

(6.3) LEMMA. Assume the above notation. Let $s, t \in T$ and write $s t=(k c) u$, where $k \in K, c \in C$ and $u \in T$. We can then write $f(s, t)=\alpha \beta$, where $\alpha$ and $\beta$ are complex numbers that are uniquely determined by the equations

(a) $v \sigma_{s} \sigma_{t}=\alpha v k \sigma_{u}$ and

(b) $w \tau_{s} \tau_{t}=\beta w c \tau_{u}$

for $v \in V$ and $w \in W$.

Observe that the elements $s, t \in T$ uniquely determine $u$ and $k c$, but that in general, $k$ and $c$ are not unique. But once the elements $k \in K$ and $c \in C$ are chosen for each pair $s, t \in T$, the equations given in (a) and (b) of Lemma 6.3 uniquely determine the scalars $\alpha$ and $\beta$, as claimed.

Proof of Lemma 6.3. By Lemma 6.1, the linear operator $\sigma_{t} \otimes \tau_{t}$ on the $K C$-module $V \otimes W$ is compatible with $t$, and thus we can use these operators to construct a well behaved quasiextension of $\theta \bullet \gamma$ with factor set $f$. By Lemma 3.5 we have

$$
f(s, t)(v \otimes w)(k c)\left(\sigma_{u} \otimes \tau_{u}\right)=(v \otimes w)\left(\sigma_{s} \otimes \tau_{s}\right)\left(\sigma_{t} \otimes \tau_{t}\right)
$$

and thus

$$
v k \sigma_{u} \otimes f(s, t)\left(w c \tau_{u}\right)=\left(v \sigma_{s} \sigma_{t}\right) \otimes\left(w \tau_{s} \tau_{t}\right)
$$

for all $v \in V$ and $w \in W$.

We can now apply Lemma 6.2 with $\mu=\sigma_{s} \sigma_{t}, \mu^{\prime}=k \sigma_{u}, \nu=\tau_{s} \tau_{t}$ and $\nu^{\prime}=f(s, t) c \tau_{u}$. We conclude that there exists a nonzero scalar $\alpha$ such that $\mu=\alpha \mu^{\prime}$ and $\nu=(1 / \alpha) \nu^{\prime}$. In particular,

$$
v \sigma_{s} \sigma_{t}=\alpha v k \sigma_{u}
$$

for all $v \in V$, as wanted. Also,

$$
w \tau_{s} \tau_{t}=(1 / \alpha) f(s, t) w c \tau_{u}
$$

for all $w \in W$, and so we take $\beta=f(s, t) / \alpha$ to complete the proof.

Before we proceed with our principal applications of Lemma 6.3, we digress to discuss the special case where $K C$ is a direct product (and not merely a central product.) 
(6.4) COROLLARY. Let $N \triangleleft G$ and suppose $N=K \times C$ is a direct product, where each of $K$ and $C$ is normal in $G$. Let $\varphi \in \operatorname{Irr}(N)$ be $G$-invariant, and write, as we may, $\varphi=\theta \gamma$, where $\theta, \gamma \in \operatorname{Irr}(N)$ and $C \subseteq \operatorname{ker}(\theta)$ and $K \subseteq \operatorname{ker}(\gamma)$. Then $[\varphi]_{G / N}=[\theta]_{G / N}[\gamma]_{G / N}$.

Note that $\theta$ and $\gamma$ are $G$-invariant, and so they do determine cohomology elements. The assertion of the corollary thus makes sense.

Proof of Corollary 6.4. Let $V$ and $W$ be $N$-modules corresponding to $\theta$ and $\gamma$, respectively, and construct well behaved quasiextensions to $G$. Fix a transversal $T$ for $N$ in $G$ and for each element $t \in T$, let $\sigma_{t}$ and $\tau_{t}$ be the linear operators determined by the quasiactions of $t$ on $V$ and $W$, respectively. Each of these operators, therefore, is compatible with $t$.

Now to put ourselves into the situation of Lemma 6.3, observe that we can identify $\theta$ and $\gamma$ with characters of $K$ and $C$ respectively, and we can view $V$ and $W$ as modules for $K$ and $C$ corresponding to these characters. From this point of view, we have $\varphi=\theta \cdot \gamma$ and Lemma 6.3 applies. Following the notation of that lemma, we can write $f(s, t)=\alpha \beta$ for $s, t \in T$, where $\alpha$ and $\beta$ are scalars determined by Equations (a) and (b) of Lemma 6.3 and depending on $s$ and $t$.

Recall that in the general situation of Lemma 6.3, the elements $k \in K$ and $c \in C$ were not uniquely determined by $s$ and $t$; only their product $k c$ was unique. Once $k$ and $c$ were fixed, however, the scalars $\alpha$ and $\beta$ were uniquely determined. In the present situation, $k$ and $c$ are determined by $k c$, and so $\alpha$ and $\beta$ are well-defined functions on $T \times T$. We can thus view $\alpha$ and $\beta$ as functions defined on $G \times G$ that are constant on cosets of $N$.

Since $C$ acts trivially on the $N$-module $V$, we can replace the element $k$ in Equation (a) of Lemma 6.3 by $k c$ (and similarly, in Equation (b), we can replace $c$ by $k c$ ). Now (a) becomes

$$
v \sigma_{s} \sigma_{t}=\alpha(s, t) v(k c) \sigma_{u},
$$

where $s t=(k c) u$. Comparison of this with the formula of Lemma 3.5 shows that the function $\alpha$ is exactly the factor set corresponding to the quasiextension $V$ of $\theta$. Similarly, $\beta$ is the factor set corresponding to $W$, and since $f=\alpha \beta$, the result follows.

As an application of Corollary 6.4, we show how it is possible to establish equality of cohomology elements of invariant characters when the factor group $G / N$ is metacyclic. (Although we will not actually use this result, it seems natural to include it.)

(6.5) THEOREM. Let $N_{1} \triangleleft G_{1}$ and $N_{2} \triangleleft G_{2}$, and let $\eta: G_{1} / N_{1} \rightarrow G_{2} / N_{2}$ be an isomorphism. Suppose that $N_{1} \subseteq C_{1} \triangleleft G_{1}$, where $C_{1} / N_{1}$ and $G_{1} / C_{1}$ are cyclic, and let $C_{1} g_{1}$ be a generator for $G_{1} / C_{1}$. Let $C_{2} / N_{2}=\eta\left(C_{1} / N_{1}\right)$ and choose an element $g_{2}$ in the coset $\eta\left(N_{1} g_{1}\right)$. Let $\theta_{i} \in \operatorname{Irr}\left(N_{i}\right)$ be $G_{i}$-invariant and choose extensions $\varphi_{i} \in \operatorname{Irr}\left(C_{i}\right)$ of $\theta_{i}$. Now $\left(\varphi_{i}\right)^{g_{i}}=\lambda_{i} \varphi_{i}$ for some uniquely determined linear character $\lambda_{i}$ of $C_{i} / N_{i}$ and we suppose that the isomorphism $\eta$ carries $\lambda_{1}$ to $\lambda_{2}$. Then $\left[\theta_{1}\right]_{G_{1} / N_{1}}=\left[\theta_{2}\right]_{G_{2} / N_{2}}$, where we identify the Schur multipliers of the groups $G_{i} / N_{i}$ via the isomorphism $\eta$.

It seems appropriate to make a few remarks. First, the extensions $\varphi_{i}$ exist since the characters $\theta_{i}$ are $C_{i}$-invariant and the groups $C_{i} / N_{i}$ are cyclic. Since $\left(\varphi_{i}\right)^{g_{i}}$ is another extension of $\theta_{i}$, it follows via Gallagher's theorem that $\left(\varphi_{i}\right)^{g_{i}}=\lambda_{i} \varphi_{i}$ for some linear character $\lambda_{i}$ as claimed. It also follows easily by Gallagher's theorem that the linear 
characters $\lambda_{i}$ do not depend on the choices of the extensions $\varphi_{i}$. Finally, we mention that $\lambda_{i}$ depends on the choice of the generating coset $C_{i} g_{i}$ but not on the specific element $g_{i}$.

We need a preliminary result that is probably well known.

(6.6) LEMMA. Let $\theta \in \operatorname{Irr}(N)$ be $G$-invariant, where $N \triangleleft G$. Then $[\bar{\theta}]_{G / N}=\left([\theta]_{G / N}\right)^{-1}$.

Proof. Recall that if $\sigma$ is a linear operator on a vector space $V$, then its adjoint $\sigma^{*}$ is the operator on the dual space $V^{*}$ defined by $\left(\sigma^{*}(\lambda)\right)(v)=\lambda(\sigma(v))$ for $v \in V$ and $\lambda \in V^{*}$.

Now let $V$ be a well behaved quasiextension of $\theta$ to $G$ with factor set $f$. We will make the dual space $V^{*}$ into a quasimodule for $G$ by letting $x \in G$ act on $V^{*}$ via the adjoint of the linear operator induced on $V$ by $x^{-1}$. In other words, we have $(\lambda \bullet x)(v)=\lambda\left(v \bullet x^{-1}\right)$ for $\lambda \in V^{*}$ and $v \in V$.

To show that this defines a quasiaction and to compute its factor set, let $x, y \in G$. Given $\lambda \in V^{*}$, we want to show that $(\lambda \cdot x) \cdot y$ is a scalar multiple of $\lambda \cdot(x y)$, and we want to find the scalar. We compute that

$$
\begin{aligned}
((\lambda \cdot x) \cdot y)(v) & =(\lambda \cdot x)\left(v \cdot y^{-1}\right) \\
& =\lambda\left(\left(v \cdot y^{-1}\right) \cdot x^{-1}\right) \\
& =f\left(y^{-1}, x^{-1}\right) \lambda\left(v \cdot\left(y^{-1} x^{-1}\right)\right) \\
& =f\left(y^{-1}, x^{-1}\right) \lambda\left(v \cdot(x y)^{-1}\right) \\
& =f\left(y^{-1}, x^{-1}\right)(\lambda \cdot(x y))(v) .
\end{aligned}
$$

This shows that indeed, our construction makes $V^{*}$ into a quasimodule and that the associated factor set $g$ is given by the formula $g(x, y)=f\left(y^{-1}, x^{-1}\right)$. In particular, $g(1, x)=1=g(x, 1)$ for all $x \in G$, and thus $V^{*}$ is a well behaved quasiextension of $V^{*}$, viewed as an $N$-module.

Since it is well known that $V^{*}$ affords the character $\bar{\theta}$ of $N$, all that remains is to show that the cohomology class of $g$ is the inverse of that of $f$ in the Schur multiplier of $G / N$. To do this, define $\alpha(x)=f\left(x, x^{-1}\right)$ and observe that

$$
\begin{aligned}
\alpha(x y) f(x, y) g(x, y) & =f\left(x y, y^{-1} x^{-1}\right) f(x, y) g(x, y) \\
& =f\left(x, y y^{-1} x^{-1}\right) f\left(y, y^{-1} x^{-1}\right) g(x, y) \\
& =\alpha(x) f\left(y, y^{-1} x^{-1}\right) f\left(y^{-1}, x^{-1}\right) \\
& =\alpha(x) f\left(y y^{-1}, x^{-1}\right) f\left(y, y^{-1}\right) \\
& =\alpha(x) \alpha(y),
\end{aligned}
$$

where the second and fourth equalities hold because $f$ is a factor set. This shows that the factor set $f g$ is cohomologically trivial on $G$. But $f, g$ and $\alpha$ are constant on cosets of $N$, and so the result follows. 
Proof of Theorem 6.5. Let $G$ be the subgroup of $G_{1} \times G_{2}$ consisting of all ordered pairs $(x, y)$ such that $\eta\left(N_{1} x\right)=N_{2} y$. Then $G$ has a normal subgroup $N=N_{1} \times N_{2}$, and (viewing the $N_{i}$ as subgroups of $G$ ) we have a natural isomorphism $G / N_{1} \cong G_{2}$ with $N / N_{1}$ mapping to $N_{2}$. (And similarly, of course, with the subscripts interchanged.) Also, since $\eta\left(C_{1} / N_{1}\right)=C_{2} / N_{2}$, there is a subgroup $C \triangleleft G$ such that $C$ maps to $C_{1}$ and to $C_{2}$ under these isomorphisms. In particular, $C_{1} / N_{1} \cong C / N \cong C_{2} / N_{2}$ is cyclic and the linear characters $\lambda_{i}$ of $C_{i} / N_{i}$ both correspond to the same linear character $\lambda$ of $C / N$. Finally, let $g=\left(g_{1}, g_{2}\right)$, so that $g \in G$ and $C g$ generates the cyclic group $G / C$.

Now view $\theta_{1}$ and $\theta_{2}$ as characters of $N$ and note that $\varphi_{1}$ and $\varphi_{2}$ can be viewed as characters of $C$ that extend $\theta_{1}$ and $\theta_{2}$, respectively. Let $\psi=\theta_{1} \overline{\theta_{2}}$ and note that $\xi=\varphi_{1} \overline{\varphi_{2}}$ is an extension of $\psi$ to $C$. It is easy to see that $\left(\varphi_{i}\right)^{g}=\lambda \varphi_{i}$, and since $\lambda \bar{\lambda}$ is trivial, it follows that $\xi^{g}=\xi$. Then $\xi$ is $G$-invariant, and as $G / C$ is cyclic, we see that $\xi$ extends to $G$. We conclude that $\psi$ extends to $G$, and so

$$
1=[\psi]_{G / N}=\left[\theta_{1}\right]_{G / N}\left[\overline{\theta_{2}}\right]_{G / N}=\left[\theta_{1}\right]_{G / N}\left(\left[\theta_{2}\right]_{G / N}\right)^{-1} .
$$

Here, of course, the second equality is a consequence of Theorem 6.4 and the third holds by Lemma 6.6. We now have $\left[\theta_{1}\right]_{G / N}=\left[\theta_{2}\right]_{G / N}$, and the result follows.

We return now to the general case. Let $G, K, C$ and $T$, be as before, where $K$ and $C$ are normal in $G$ and centralize each other, and $T$ is a transversal for the cosets of $K C$ in $G$. Also let $\theta, \gamma, V$ and $W$ be as before, where $\theta \in \operatorname{Irr}(K)$ and $\gamma \in \operatorname{Irr}(C)$ are $G$-invariant, and where $V$ and $W$ are modules affording $\theta$ and $\gamma$, respectively. Recall that we are assuming that $\theta$ and $\gamma$ both lie over a common linear character $\nu$ of $Z=K \cap C$, and thus $\theta \cdot \gamma \in \operatorname{Irr}(K C)$ is defined.

Fix a well behaved (with respect to the action of $K$ ) quasiaction of $G$ on $V$, and let $h$ be the associated factor set. (Recall that we can choose to view $h$ either as a factor set of $G$, constant on cosets of $K$, or as a factor set of $G / K$.) In Lemma 6.3, choose the operators $\sigma_{t}$ on $V$ to be the maps $v \mapsto v \bullet t$ for $t \in T$ and choose arbitrary operators $\tau_{t}$ of $W$ compatible with $t \in T$. As in Lemma 6.3, we use the maps $\sigma_{t}$ and $\tau_{t}$, to construct a quasiextension of the $K C$-module $V \otimes W$, with factor set $f$. Our next goal is to compute the factor set $h / f$ of $G$ using as little information as possible. (Note that $h$ lies in the cohomology class $[\theta]_{G / K}$ and $f$ lies in the cohomology class $[\theta \cdot \gamma]_{G / K C}$, but both can be viewed as factor sets of $G$ or of $G / K$, and so $h / f$ can be viewed as a factor set of either $G$ or $G / K$.)

Given the transversal $T$ for the cosets of $K C$ in $G$, we know that for $s, t \in T$, there are uniquely determined elements $u \in T$ and $n \in K C$ such that $s t=n u$. We view $n$ and $u$ as functions from $T \times T$ into $K C$ and into $T$ respectively, and we write $n=n(s, t)$ and $u=u(s, t)$. Also, we recall that the $C$-type of the $G$-quasiextension $V$ of $\theta$ consists of two complex-valued functions. One of these is $\rho$, defined on $C$ and determined by the formula $v \bullet c=\rho(c) v$ for $v \in V$. The second component of the $C$-type of $V$ is the factor set $h$ corresponding to $V$, with the first variable restricted to $C$.

We can now state our result. 
(6.7) THEOREM. In the above situation, the factor set $h / f$ of $G$ is determined by the choice of the transversal $T$, the group $C$, the automorphisms of $C$ induced by the elements of $T$, the functions $n$ and $u$ on $T \times T$, the $C$-module $W$, the operators $\tau_{t}$ on $W$ for $t \in T$, the linear character $\nu$ of $Z$, and the $C$-type of the $G$-quasimodule $V$.

What we do not need to know is the actual $G$-quasimodule $V$ or its factor set $h$. To some extent, even the group $K$ is irrelevant, as in the following.

(6.8) COROLLARY. Let $K \triangleleft G$ and $C \triangleleft G$, with $[K, C]=1$, and suppose that $C \subseteq$ $N \subseteq G$ and $K N=G$. Let $H=K \cap N$ and $Z=K \cap C$, so that $Z \subseteq H$ and $Z \subseteq \mathbf{Z}(K)$. Let $\theta \in \operatorname{Irr}(K)$ and $\gamma \in \operatorname{Irr}(C)$ be $G$-invariant and lie over a linear character $\nu$ of $Z$, and let $\theta^{\prime} \in \operatorname{Irr}(H)$ also lie over $\nu$, where $\theta^{\prime}$ is $N$-invariant. Suppose that $V$ and $V^{\prime}$ are well behaved quasiextensions of $\theta$ and $\theta^{\prime}$ to $G$ and $N$ that have the same $C$-type, and let $h$ and $h^{\prime}$ be the corresponding factor sets of $G / K$ and $N / H$, which we identify. Then there exist factor sets $f$ and $f^{\prime}$ of $G / K C=N / H C$ lying in the cohomology classes $[\theta \cdot \gamma]_{G / K C}$ and $\left[\theta^{\prime} \cdot \gamma\right]_{N / H C}$, respectively, such that $h / f=h^{\prime} / f^{\prime}$ as factor sets of $G / K=N / H$.

Proof. Choose a transversal $T$ for the cosets of $H C$ in $N$ and observe that $T$ is also a transversal for $K C$ in $G$. Of course, in this situation, the function $n: T \times T \rightarrow K C$ actually maps into $H C$. Since we can use the same operators $\tau_{t}$ on $W$ to compute the factor sets $f$ and $f^{\prime}$, the result follows via Theorem 6.7.

We mention that the assumption in Corollary 6.8 that the quasiextensions $V$ and $V^{\prime}$ of $\theta$ and $\theta^{\prime}$ have identical $C$-types is not a burden. By Lemma 3.7, such quasiextensions necessarily exist.

Proof of Theorem 6.7. Given an arbitrary element $x \in G$, write $\sigma_{x}$ to denote the linear operator $v \mapsto v \bullet x$ on $V$. (We have already defined $\sigma_{t}$ for $t \in T$; this simply extends that definition.)

If $c \in C$, then by definition of the factor set $h$, we have $h(c, t) \sigma_{c t}=\sigma_{c} \sigma_{t}$. Also, since $c \in C$, we know that the operator $\sigma_{c}$ is simply multiplication by some scalar $\rho(c)$, and we have $\sigma_{c t}=(\rho(c) / h(c, t)) \sigma_{t}$. Since the element $c t \in C T$ uniquely determines $c$ and $t$, we can define the function $\delta$ on the subset $C T$ by $\delta(c t)=\rho(c) / h(c, t)$, and we have $\sigma_{c t}=\delta(c t) \sigma_{c}$. The key observation here is that $\delta$ depends only on the transversal $T$ and the $C$-type of $V$. In particular, it is determined by the given information.

Fix a transversal $R$ for $Z=K \cap C$ in $C$, and observe that $R T$ is a transversal for $K$ in $G$. We want to use Lemma 3.5 with the transversal $R T$ to compute the factor set $h$. To do this, let $p s, q t \in R T$, where $p, q \in R$ and $s, t \in T$. We can write $(p s)(q t)=l(r u)$, where $l \in K, r \in R$ and $u \in T$. Also, $(p s)(q t)$ lies in the same coset of $K C$ as $s t$, and thus $u=u(s, t)$ and we can write $s t=k c u$, where $k \in K, c \in C$ and $k c=n(s, t)$. (As usual, $k c$ is uniquely determined by $s$ and $t$, but $k$ and $c$ need not be. We can, however, fix a choice of $k$ and $c$ for each pair $s, t \in T$.)

Now

$$
l(r u)=(p s)(q t)=p q^{s^{-1}} s t=p q^{s^{-1}} k c u .
$$

Canceling $u$ and using the fact that $K$ and $C$ centralize each other, we see that $l k^{-1}=$ $p q^{s^{-1}} r^{-1} \in K \cap C=Z$. Furthermore, the element $l k^{-1}$ (depending on $s, t \in T$ ) is determined by the given data and the choices we have made. 
By Lemma 3.5, we have

$$
h(p s, q t) v l \sigma_{r u}=v \sigma_{p s} \sigma_{q t}
$$

for $v \in V$. Since $\sigma_{r u}=\delta(r u) \sigma_{u}$ and similarly for $\sigma_{p s}$ and $\sigma_{q t}$, we can rewrite this equation as

$$
h(p s, q t) \delta(r u) v l \sigma_{u}=\delta(p s) \delta(q t) v \sigma_{s} \sigma_{t} .
$$

By Lemma 6.3, we can write $f(s, t)=\alpha \beta$ where

(a) $v \sigma_{s} \sigma_{t}=\alpha v k \sigma_{u}$ and

(b) $w \tau_{s} \tau_{t}=\beta w c \tau_{u}$

for all $v \in V$ and $w \in W$. In particular the scalar $\beta$ (depending on $s$ and $t$ ) is determined from the given data and our choices.

This yields

$$
h(p s, q t) \delta(r u) v l \sigma_{u}=\delta(p s) \delta(q t) \alpha v k \sigma_{u},
$$

and thus since $l k^{-1}$ acts on $V$ via the scalar $\nu\left(l k^{-1}\right)$, we have

$$
h(p s, q t) \delta(r u) \nu\left(l k^{-1}\right)=\alpha \delta(p s) \delta(q t) .
$$

Finally, since $f(s, t)=\alpha \beta$, we obtain the formula

$$
h(p s, q t)=\frac{\alpha \delta(p s) \delta(q t)}{\delta(r u) \nu\left(l k^{-1}\right)}=f(s, t) \frac{\delta(p s) \delta(q t)}{\beta \delta(r u) \nu\left(l k^{-1}\right)} .
$$

Now $f$ is constant on cosets of $K C$ in $G$, and so $f(s, t)=f(p s, q t)$, and we deduce that

$$
\frac{h(p s, q t)}{f(p s, q t)}=\frac{\delta(p s) \delta(q t)}{\beta \delta(r u) \nu\left(l k^{-1}\right)} .
$$

Each factor on the right side of this equation is determined by the given data and the choices we have made, and so the result follows.

In the previous proof, we chose the transversal $R$ and for each pair $s, t \in T$, we chose elements $k \in K$ and $c \in C$ so that $k c=n(s, t)$. It is perhaps worrisome that the calculation of the factor set ratio $h / f$ appears to depend on these arbitrary choices, since neither $h$ nor $f$ is dependent on them. In fact, a little thought shows that the dependence is illusory. Suppose, for example, that $p$ is replaced by another representative $z p$ of the coset $Z p$. This has the effect of multiplying $\delta(p s)$ by $\nu(z)$. But another consequence is that the element $l k^{-1}$ is multiplied by $z$, and so there is no net effect. Similarly, there is no effect if $q$ and $r$ are replaced by other representatives of their cosets. Also, $k$ could be changed to $k z$, where $z \in Z$, but then $c$ would become $c z^{-1}$ and this would change $\beta$ to $\beta \nu(z)$, and again there would be no net effect. 


\section{Related characters.}

In order to apply the theory of the previous sections, we make some definitions. Suppose $Z \subseteq H \subseteq K$, where $Z \subseteq \mathbf{Z}(K)$, and let $\nu \in \operatorname{Irr}(Z)$. In this situation, we say that $(K, H, \nu)$ is an admissible triple. (We suppress explicit mention of $Z$ in this notation since $Z$ is determined implicitly by $\nu$ : it is the domain of the linear character $\nu$.) We shall say that a pair of characters $\theta \in \operatorname{Irr}(K)$ and $\theta^{\prime} \in \operatorname{Irr}(H)$ belongs to the admissible triple $(K, H, \nu)$ if both $\theta$ and $\theta^{\prime}$ lie over $\nu$.

In the above situation, suppose that $K$ is a normal subgroup of some group $G$, and let $N \subseteq G$ be a subgroup such that $K N=G$ and $K \cap N=H$. Let $C \triangleleft G$ and assume that $[K, C]=1$, that $C \subseteq N$ and that $C \cap K=Z$. Under all of these conditions, we say that $(G, N, C)$ lies over the admissible triple $(K, H, \nu)$. Note that $H \triangleleft N$ in this situation, and thus $N$ acts on the set $\operatorname{Irr}(H)$.

Let $(G, N, C)$ lie over the admissible triple $(K, H, \nu)$ and suppose that $\left(\theta, \theta^{\prime}\right)$ is a pair of characters that belongs to $(K, H, \nu)$. We are especially interested in the case where $\theta$ is $G$-invariant and $\theta^{\prime}$ is $N$-invariant, and in this situation, we simply say that the pair $\left(\theta, \theta^{\prime}\right)$ is invariant in $(G, N, C)$. (Note that if $\theta$ is $N$-invariant, it is automatically $G$-invariant since $K N=G$.)

It is convenient to restate Lemma 3.7 in this language. The lemma says that if $\left(\theta, \theta^{\prime}\right)$ belongs to $(K, H, \nu)$ and is invariant in $(G, N, C)$, which lies over $(K, H, \nu)$, then there exist well behaved quasiextensions $V$ and $V^{\prime}$ of $\theta$ and $\theta^{\prime}$ to $G$ and $N$ such that $V$ and $V^{\prime}$ have identical $C$-types.

We can also restate Corollary 6.8 in this situation. Let $h$ and $h^{\prime}$ be the factor sets associated with $V$ and $V^{\prime}$, and assume that there exists a character $\gamma \in \operatorname{Irr}(C)$ that lies over $\nu$ and is $G$-invariant. Then 6.8 asserts that there exist factor sets $f$ and $f^{\prime}$ in the cohomology classes $[\theta \cdot \gamma]_{G / K C}$ and $\left[\theta^{\prime} \cdot \gamma\right]_{N / H C}$ such that $h / f=h^{\prime} / f^{\prime}$.

One of our goals is to find conditions that guarantee that the cohomology elements $[\theta \cdot \gamma]_{G / K C}$ and $\left[\theta^{\prime} \cdot \gamma\right]_{N / H C}$ are equal in the above situation. Specifically, given that $\left(\theta, \theta^{\prime}\right)$ belongs to $(K, H, \nu)$ and is invariant in $(G, N, C)$, which lies over $(K, H, \nu)$, we would like to have $[\theta \cdot \gamma]_{G / K C}=\left[\theta^{\prime} \cdot \gamma\right]_{N / H C}$, where $\gamma \in \operatorname{Irr}(C \mid \nu)$ is $G$-invariant. If this equality of cohomology elements holds, we will say that $\theta$ and $\theta^{\prime}$ are related in $(G, N, C)$ with respect to $\gamma$.

We investigate what happens when $C$ is replaced by a smaller subgroup $D$. Note that if $(G, N, C)$ lies over $(K, H, \nu)$ and $Z \subseteq D \subseteq C$ with $D \triangleleft G$, then $(G, N, D)$ also lies over $(K, H, \nu)$. (Here, as usual, $Z$ is the domain of $\nu$.)

(7.1) THEOREM (Going Down). Let $\left(\theta, \theta^{\prime}\right)$ belong to $(K, H, \nu)$ and let $Z$ be the domain of $\nu$. Suppose that $(G, N, C)$ lies over $(K, H, \nu)$ and that $\left(\theta, \theta^{\prime}\right)$ is invariant in $(G, N, C)$. Let $\gamma \in \operatorname{Irr}(C \mid \nu)$ be $G$-invariant, and assume that $\theta$ and $\theta^{\prime}$ are related in $(G, N, C)$ with respect to $\gamma$. Suppose that $Z \subseteq D \subseteq C$, where $D \triangleleft G$, and let $\tau \in \operatorname{Irr}(D \mid \nu)$ be $G$-invariant. Then $\theta$ and $\theta^{\prime}$ are related in $(G, N, D)$ with respect to $\tau$.

Observe that Theorem 7.1 has content even when $D=C$. In that case, the theorem asserts that the $G$-invariant character $\gamma \in \operatorname{Irr}(C \mid \nu)$ can be replaced by any other $G$-invariant character $\tau$ in this set without affecting the equality of the appropriate cohomology elements. In other words, the equality $[\theta \cdot \gamma]_{G / K C}=\left[\theta^{\prime} \cdot \gamma\right]_{N / H C}$ is really an assertion about the pair $\left(\theta, \theta^{\prime}\right)$; the specific $G$-invariant character $\gamma \in \operatorname{Irr}(C)$ is irrelevant. 
If $\left(\theta, \theta^{\prime}\right)$ belongs to $(K, H, \nu)$ and is invariant in $(G, N, C)$, which lies over $(K, H, \nu)$, we say that the characters $\theta$ and $\theta^{\prime}$ are related in $(G, N, C)$ if $\theta$ and $\theta^{\prime}$ are related with respect to some (and hence every) choice of a $G$-invariant character $\gamma \in \operatorname{Irr}(C \mid \nu)$.

Of course, it does not make sense to ask if $\theta$ and $\theta^{\prime}$ are related in $(G, N, C)$ unless the pair $\left(\theta, \theta^{\prime}\right)$ is invariant in $(G, N, C)$ and there exists at least one $G$-invariant character $\gamma \in \operatorname{Irr}(C \mid \nu)$. In this situation, we shall say that $(G, N, C)$ is a relatedness candidate for $\left(\theta, \theta^{\prime}\right)$. We can thus paraphrase Theorem 7.1 like this: if $\theta$ and $\theta^{\prime}$ are related in $(G, N, C)$ and $(G, N, D)$ is a relatedness candidate with $Z \subseteq D \subseteq C$, then $\theta$ and $\theta^{\prime}$ are related in $(G, N, D)$.

Proof of Theorem 7.1. Construct quasimodules $V$ and $V^{\prime}$ of $G$ and $N$ extending $\theta$ and $\theta^{\prime}$, and do this so that $V$ and $V^{\prime}$ have identical $C$-types. (Recall that this is possible by Lemma 3.7.) Let $h$ and $h^{\prime}$ be the factor sets associated with $V$ and $V^{\prime}$, and view them as factor sets of $G / K$ and $N / H$, which we identify. By Corollary 6.8, we know that there exist factor sets $f$ and $f^{\prime}$ of $G / K=N / H$ with the following properties

(a) $f$ and $f^{\prime}$ are constant on cosets of $K C / K=H C / H$

(b) $f$ and $f^{\prime}$ are in the cohomology classes $[\theta \cdot \gamma]_{G / K C}$ and $\left[\theta^{\prime} \cdot \gamma\right]_{N / H C}$ respectively, and

(c) $h / f=h^{\prime} / f^{\prime}$ as factor sets of $G / K=N / H$.

In particular, we have $h / h^{\prime}=f / f^{\prime}$.

Since $D \subseteq C$, the quasimodules $V$ and $V^{\prime}$ also have identical $D$-types, and so again by Corollary 6.8, we have $h / h^{\prime}=g / g^{\prime}$, where $g$ and $g^{\prime}$ lie in the cohomology classes $[\theta \cdot \tau]_{G / K D}$ and $\left[\theta^{\prime} \cdot \tau\right]_{N / H D}$, and we are viewing $g$ and $g^{\prime}$ as factor sets of $G / K$ and $N / H$ that are constant on cosets of $K D / K=H D / H$.

We conclude that $f / f^{\prime}=g / g^{\prime}$, where we view all four of these functions as factor sets of $G / K$, where $f$ and $f^{\prime}$ are constant on cosets of $K C / K$ in this group, and $g$ and $g^{\prime}$ are constant on cosets of $K D / K$. By hypothesis, $f$ and $f^{\prime}$ are cohomologous when they are viewed as factor sets of $G / K C$. In other words, $f / f^{\prime}$ is a function of the form $\alpha(x) \alpha(y) / \alpha(x y)$, where $\alpha$ is a function defined on $G / K$ that is constant on cosets of $K C / K$ in $G / K$. But $f / f^{\prime}=g / g^{\prime}$, and since $D \subseteq C$, we see that $\alpha$ is constant on cosets of $K D / K$ in $G / K$. It follows that $g$ and $g^{\prime}$ are cohomologous when viewed as factor sets of $G / K D$.

The following rather technical theorem is an essential step in obtaining our main result.

(7.2) THEOREM. Let $\left(\theta, \theta^{\prime}\right)$ belong to $(K, H, \nu)$ and suppose that $(G, N, C)$ is a relatedness candidate for this pair of characters. Let $\beta \in \operatorname{Irr}(K)$ and $\beta^{\prime} \in \operatorname{Irr}(H)$ and assume that each of these characters has stabilizer $N_{0}$ in $N$, and that each of them lies over $\lambda \in \operatorname{Irr}(Z)$, where $Z$ is the domain of $\nu$. Assume, furthermore, that $\theta$ is the product of the $\left|N: N_{0}\right|$ distinct $N$-conjugates of $\beta$ and that $\theta^{\prime}$ is the product of the $\left|N: N_{0}\right|$ distinct $N$-conjugates of $\beta^{\prime}$. Write $G_{0}=K N_{0}$ and note that $\left(G_{0}, N_{0}, C\right)$ lies over $(K, H, \lambda)$. If $\beta$ and $\beta^{\prime}$ are related in $\left(G_{0}, N_{0}, C\right)$, then $\theta$ and $\theta^{\prime}$ are related in $(G, N, C)$.

Proof. Observe that $\left(\beta, \beta^{\prime}\right)$ belongs to $(K, H, \lambda)$ and is invariant in $\left(G_{0}, N_{0}, C\right)$. Choose well behaved quasimodules $V$ and $V^{\prime}$ of $G_{0}$ and $N_{0}$ corresponding to $\beta$ and $\beta^{\prime}$ and having the same $C$-type, and let $g$ and $g^{\prime}$ be their factor sets, viewed as factor sets of $G_{0} / K=$ 
$N_{0} / H$. Also, since we are assuming that $\beta$ and $\beta^{\prime}$ are related in $\left(G_{0}, N_{0}, C\right)$, we know that there exists a $G_{0}$-invariant character $\tau \in \operatorname{Irr}(C \mid \lambda)$ such that $[\beta \bullet \tau]_{G_{0} / K C}=\left[\beta^{\prime} \cdot \tau\right]_{N_{0} / H C}$.

Let $j$ and $j^{\prime}$ be factor sets of $G_{0} / K=N_{0} / H$ that are constant on cosets of $K C / K=$ $H C / H$ and such that when viewed as factor sets of $G_{0} / K C=N_{0} / H C$, they lie in the cohomology classes $[\beta \cdot \tau]_{G_{0} / K C}$ and $\left[\beta^{\prime} \cdot \tau\right]_{N_{0} / H C}$ respectively. By Corollary 6.8 , the factor sets $j$ and $j^{\prime}$ can be chosen so that $g / j=g^{\prime} / j^{\prime}$. Also, since we are assuming that $[\beta \cdot \tau]_{G_{0} / K C}=\left[\beta^{\prime} \cdot \tau\right]_{N_{0} / H C}$, it follows that $j$ and $j^{\prime}$ are cohomologous as factor sets of $G_{0} / K C=N_{0} / H C$.

By the results of Section 4, the tensor-induced quasimodules $V^{\otimes G}$ and $\left(V^{\prime}\right)^{\otimes N}$ are well behaved quasiextensions of $\theta$ and $\theta^{\prime}$ that have identical $C$-types. Let $h$ and $h^{\prime}$ be the corresponding factor sets of $G / K=N / H$, so that we have $h=g^{G / K}$ and $h^{\prime}=\left(g^{\prime}\right)^{N / H}$.

By assumption, $(G, N, C)$ is a relatedness candidate for $\left(\theta, \theta^{\prime}\right)$, and so in particular, there exists a $G$-invariant character $\gamma \in \operatorname{Irr}(C \mid \nu)$. Let $f$ and $f^{\prime}$ be factor sets of $G / K=$ $N / H$ that are constant on cosets of $K C / K=H C / H$ and such that when viewed as factor sets of $G / K C$ and $N / H C$, they lie in the cohomology classes $[\theta \cdot \gamma]_{G / K C}$ and $\left[\theta^{\prime} \cdot \gamma\right]_{N / H C}$, respectively. By Corollary 6.8, we can choose $f$ and $f^{\prime}$ so that $h / f=h^{\prime} / f^{\prime}$. Our goal is to show that $f$ and $f^{\prime}$ are cohomologous as factor sets of $G / K C=N / H C$.

We have $h=g^{G / K}$ and $h^{\prime}=\left(g^{\prime}\right)^{N / H}$. Identifying $G / K=N / H$ and using the fact that factor-set induction respects multiplication of factor sets, we can write

$$
\frac{h}{h^{\prime}}=\left(\frac{g}{g^{\prime}}\right)^{G / K} .
$$

Since $h / h^{\prime}=f / f^{\prime}$ and $g / g^{\prime}=j / j^{\prime}$, we have

$$
\frac{f}{f^{\prime}}=\left(\frac{j}{j^{\prime}}\right)^{G / K} .
$$

Now $j / j^{\prime}$ is a factor set of $G_{0} / K$ that is constant on cosets of the subgroup $K C / K$. It follows that we can view $j / j^{\prime}$ as a factor set of $G_{0} / K C$ and furthermore, the induction to $G / K$ can be viewed as induction to $G / K C$. But by hypothesis, $j / j^{\prime}$ is cohomologously trivial as a factor set of $G_{0} / K C$, and thus induction to $G / K C$ yields a factor set of $G / K C$ that is also cohomologously trivial. This shows that $f$ and $f^{\prime}$ are cohomologous as factor sets of $G / K C=N / H C$, as required.

\section{Equivalence.}

Let $(K, H, \nu)$ be an admissible triple. (Recall that this means that $\nu \in \operatorname{Irr}(Z)$, where $Z \subseteq H \subseteq K$ and $Z \subseteq \mathbf{Z}(K)$.) We will say that $(K, H, \nu)$ is self-normalizing if $H=$ $\mathbf{N}_{K}(H)$. If $(G, N, C)$ lies over $(K, H, \nu)$, then, by definition, $H=K \cap N$, and thus $H \triangleleft N$. If $(K, H, \nu)$ happens to be self-normalizing, however, we can say more. In this case $N=\mathbf{N}_{G}(H)$. Also, the condition that $C \subseteq N$ in the definition of "lies over" is redundant if $(K, H, \nu)$ is self-normalizing because in that case, $C \subseteq \mathbf{C}_{G}(K) \subseteq \mathbf{N}_{G}(H)=N$. Later we will need one further condition, which for convenience we describe now: we say that a self-normalizing admissible triple is strong if $Z=\mathbf{Z}(K)$. 
Suppose that both $\left(G_{1}, N_{1}, C_{1}\right)$ and $\left(G_{2}, N_{2}, C_{2}\right)$ lie over $(K, H, \nu)$, which we assume to be self-normalizing. Note that since $C_{i}$ centralizes $K$ for $i \in\{1,2\}$, all of the elements in each coset of $C_{i}$ in $G_{i}$ induce the same automorphism of $K$. We will say that the triples $\left(G_{1}, N_{1}, C_{1}\right)$ and $\left(G_{2}, N_{2}, C_{2}\right)$ are equivalent over $(K, H, \nu)$ if there exists an isomorphism $\eta: G_{1} / C_{1} \rightarrow G_{2} / C_{2}$ such that

(1) $\eta\left(C_{1} k\right)=C_{2} k$ for all $k \in K$ and

(2) If $\eta\left(C_{1} x\right)=C_{2} y$, then $x$ and $y$ induce the same automorphism of $K$. (In other words $k^{x}=k^{y}$ for all $k \in K$.)

We say that $\eta$ is an equivalence in this situation.

(8.1) LEMMA. Suppose that $\left(G_{1}, N_{1}, C_{1}\right)$ and $\left(G_{2}, N_{2}, C_{2}\right)$ are equivalent over a selfnormalizing admissible triple $(K, H, \nu)$, and that $\eta$ is an equivalence. Then $\eta$ maps $N_{1} / C_{1}$ onto $N_{2} / C_{2}$. Also if $\varphi$ is an irreducible character of either $K$ or $H$, then $\varphi$ is $N_{1}$-invariant if and only if $\varphi$ is $N_{2}$-invariant.

Proof. Suppose $\eta\left(C_{1} x\right)=C_{2} y$. Then $x$ and $y$ induce the same automorphism of $K$, and so $x$ stabilizes $H$ if and only if $y$ stabilizes $H$. Since $N_{i}=\mathbf{N}_{G_{i}}\left(H_{i}\right)$, it follows that $x \in N_{1}$ if and only if $y \in N_{2}$. The first assertion now follows.

Now assume that $x \in N_{1}$ and $y \in N_{2}$. Then since $x$ and $y$ induce the same automorphism on $K$, and hence also on $H$, we see that $x$ stabilizes $\varphi$ if and only if $y$ stabilizes $\varphi$. This completes the proof.

In particular, in the situation of Lemma 8.1, if $\left(\theta, \theta^{\prime}\right)$ belongs to $(K, H, \nu)$, then this pair of characters is invariant in $\left(G_{1}, N_{1}, C_{1}\right)$ if and only if it is invariant in $\left(G_{2}, N_{2}, C_{2}\right)$.

If $\eta$ is an equivalence between $\left(G_{1}, N_{1}, C_{1}\right)$ and $\left(G_{2}, N_{2}, C_{2}\right)$, then $\eta$ maps $K C_{1} / C_{1}$ to $K C_{2} / C_{2}$, and hence it induces an isomorphism from $G_{1} / K C_{1}$ onto $G_{2} / K C_{2}$. Also, these groups are naturally isomorphic to $N_{1} / H C_{1}$ and $N_{2} / H C_{2}$, and so we can identify these four factor groups for the purpose of comparing cohomology elements.

(8.2) THEOREM. Let $(K, H, \nu)$ be self-normalizing and suppose that $\left(G_{1}, N_{1}, C_{1}\right)$ and $\left(G_{2}, N_{2}, C_{2}\right)$ are equivalent over it. Suppose that $\left(\theta, \theta^{\prime}\right)$ belongs to $(K, H, \nu)$ and is invariant in $\left(G_{i}, N_{i}, C_{i}\right)$, and assume that $\gamma_{i} \in \operatorname{Irr}\left(C_{i} \mid \nu\right)$ is $G_{i}$-invariant for $i \in\{1,2\}$. Then

$$
\frac{\left[\theta \cdot \gamma_{1}\right]_{G_{1} / K C_{1}}}{\left[\theta^{\prime} \cdot \gamma_{1}\right]_{N_{1} / H C_{1}}}=\frac{\left[\theta \cdot \gamma_{2}\right]_{G_{2} / K C_{2}}}{\left[\theta^{\prime} \cdot \gamma_{2}\right]_{N_{2} / H C_{2}}}
$$

Proof. Choose a transversal $T_{1}$ for $H C_{1}$ in $N_{1}$ and note that $T_{1}$ is also a transversal for $K C_{1}$ in $G_{1}$. We appeal to Lemma 6.3 to compute factor sets $f$ and $f^{\prime}$ associated with well behaved quasiextensions of $\theta \cdot \gamma_{1}$ to $G_{1}$ and $\theta^{\prime} \cdot \gamma_{1}$ to $N_{1}$, respectively. We use the same transversal $T_{1}$ to compute both $f$ and $f^{\prime}$, and also we use the same $C_{1}$-module $W$ affording $\gamma_{1}$ and the same linear operators $\tau_{t}$ on $W$, where $\tau_{t}$ is compatible with $t \in T_{1}$.

Given $s, t \in T_{1}$, write $s t=k c_{1} u$, where $k \in K, c_{1} \in C_{1}$ and $u \in T_{1}$. Then $k \in N_{1} \cap K=$ $H$, and in particular, we can use the same elements $k$ and $c_{1}$ in the computations of both $f$ and $f^{\prime}$. It follows that the scalar $\beta$ of Lemma 6.3 (depending on $s$ and $t$ ) is the same in both computations. We thus have $f(s, t)=\alpha \beta$ and $f^{\prime}(s, t)=\alpha^{\prime} \beta$, where $\alpha$ and $\alpha^{\prime}$ are as in Lemma 6.3, and hence $f(s, t) / f^{\prime}(s, t)=\alpha / \alpha^{\prime}$. 
To compute $\alpha$, we work with certain linear operators $\sigma_{t}$ on a module $V$ affording $\theta$, where $\sigma_{t}$ is compatible with $t \in T_{1}$. These operators are not uniquely determined, but the compatibility conditions that they are required to satisfy depend only on the conjugation actions of the elements of $T_{1}$ on $K$ and the actions of the elements of $K$ on the $K$-module $V$.

Once we have chosen the operators $\sigma_{t}$ on $V$ for all $t \in T_{1}$, the scalar $\alpha$ is determined by the equation $v \sigma_{s} \sigma_{t}=\alpha v k \sigma_{u}$, where $k \in K$ is as above. The scalar $\alpha^{\prime}$ is determined similarly, with the various operators $\sigma$ on $V$ replaced by operators $\sigma^{\prime}$ on an $H$-module $V^{\prime}$ affording $\theta^{\prime}$, but using the same elements $k$.

Now let $\eta$ be an equivalence from $\left(G_{1}, N_{1}, C_{1}\right)$ to $\left(G_{2}, N_{2}, C_{2}\right)$. For each element $t \in T_{1}$, we know that $\eta\left(C_{1} t\right)$ is a coset of $C_{2}$ in $G_{2}$, and we choose an arbitrary element of this coset. Let $T_{2}$ be the set of elements chosen in this way, one for each element of $T_{1}$. In order to make this clearer, we change notation slightly at this point: if $t_{1}$ is an element of $T_{1}$, we write $t_{2}$ to denote the corresponding element of $T_{2}$. Thus $\eta\left(C_{1} t_{1}\right)=C_{2} t_{2}$ for all elements $t_{1} \in T_{1}$.

Recall that the isomorphism $\eta$ from $G_{1} / C_{1}$ onto $G_{2} / C_{2}$ maps $K C_{1} / C_{1}$ to $K C_{2} / C_{2}$, and thus it induces an isomorphism from $G_{1} / K C_{1}$ onto $G_{2} / K C_{2}$. The group $G_{1} / K C_{1}$ is exactly the set of cosets $K C_{1} t_{1}$ as $t_{1}$ runs over $T_{1}$, and these cosets are distinct. Under the isomorphism from $G_{1} / K C_{1}$ to $G_{2} / K C_{2}$, the coset $K C_{1} t_{1}$ maps to $K C_{2} t_{2}$, and thus the cosets $K C_{2} t_{2}$ are distinct as $t_{1}$ runs over $T_{1}$. It follows that the set $T_{2}$ is a transversal for the cosets of $K C_{2}$ in $G_{2}$. (And in particular, the elements $t_{2}$ are distinct for distinct elements $t_{1} \in T_{1}$.)

Because $\eta$ is an equivalence, we know that $t_{1}$ and $t_{2}$ induce identical automorphisms of $K$. Also, $t_{1} \in N_{1}$, and so $t_{1}$ stabilizes $H$. It follows that $t_{2} \in \mathbf{N}_{G_{2}}(H)=N_{2}$, and thus $\mathrm{T}_{2}$ is a transversal for the cosets of $\mathrm{HC}_{2}$ in $\mathrm{N}_{2}$.

If we compute factor sets $g$ and $g^{\prime}$ corresponding to well behaved quasiextensions of $\theta \cdot \gamma_{2}$ and $\theta^{\prime} \cdot \gamma_{2}$ to $G_{2}$ and $N_{2}$ using the transversal $T_{2}$, we find that $g\left(s_{2}, t_{2}\right) / g^{\prime}\left(s_{2}, t_{2}\right)=$ $\alpha / \alpha^{\prime}$, where we will show that the scalars $\alpha$ and $\alpha^{\prime}$ are the same as those that we had in the computation of $f\left(s_{1}, t_{1}\right) / f^{\prime}\left(s_{1}, t_{1}\right)$.

For each element $t_{1} \in T_{1}$, we had a corresponding compatible linear operator $\sigma: V \rightarrow$ $V$. We observe that since the conjugation actions of $t_{1}$ and $t_{2}$ on $K$ are identical, the operator $\sigma$, which was chosen to be compatible with $t_{1}$, will also be compatible with $t_{2}$. In other words, we can use exactly the same linear operators $\sigma$ to compute the factor set $g$ that we used previously to compute $f$.

The ingredients used to compute the scalar $\alpha$ in Lemma 6.3 were the various linear operators $\sigma$ on $V$ associated with the elements of the transversal and also the elements $k \in K$ chosen for pairs of elements of the transversal. Suppose then, that $s_{1}, t_{1} \in T_{1}$, and that we have written $s_{1} t_{1}=k c_{1} u_{1}$, where $c_{1} \in C_{1}, k \in K$ and $u_{1} \in T_{1}$. Recalling that $\eta\left(C_{1} k\right)=C_{2} k$, we compute

$$
\begin{aligned}
C_{2} s_{2} t_{2}=\left(C_{2} s_{2}\right)\left(C_{2} t_{2}\right)=\eta\left(C_{1} s_{1}\right) \eta\left(C_{1} t_{1}\right) & =\eta\left(C_{1} s_{1} t_{1}\right) \\
& =\eta\left(C_{1} k u_{1}\right) \\
& =\eta\left(C_{1} k\right) \eta\left(C_{1} u_{1}\right) \\
& =\left(C_{2} k\right)\left(C_{2} u_{2}\right)=C_{2} k u_{2} .
\end{aligned}
$$


We can thus write $s_{2} t_{2}=c_{2} k u_{2}$ for some element $c_{2} \in C_{2}$, and we see that when computing the factor set $g$, we can use the same element $k$ that we used previously, when we computed the factor set $f$. It follows that the computations of $f\left(s_{1}, t_{1}\right)$ and $g\left(s_{2}, t_{2}\right)$ involve exactly the same scalar $\alpha$. Similarly, the computations of $f^{\prime}\left(s_{1}, t_{1}\right)$ and $g^{\prime}\left(s_{2}, t_{2}\right)$ involve the same scalar $\alpha^{\prime}$, and hence $f / f^{\prime}=g / g^{\prime}$, as required.

(8.3) COROLLARY. Suppose that $\left(\theta, \theta^{\prime}\right)$ belongs to $(K, H, \nu)$, where $(K, H, \nu)$ is selfnormalizing. Let $\left(G_{1}, N_{1}, C_{1}\right)$ and $\left(G_{2}, N_{2}, C_{2}\right)$ be equivalent relatedness candidates for $\left(\theta, \theta^{\prime}\right)$ over $(K, H, \nu)$. Then $\theta$ and $\theta^{\prime}$ are related in $\left(G_{1}, N_{1}, C_{1}\right)$ if and only if they are related in $\left(G_{2}, N_{2}, C_{2}\right)$.

Proof. Since the ratios in the statement of Theorem 8.2 are equal, it follows that the ratio on the left equals 1 if and only if the ratio on the right equals 1 . In other words, $\theta$ and $\theta^{\prime}$ are related in $\left(G_{1}, N_{1}, C_{1}\right)$ if and only if they are related in $\left(G_{2}, N_{2}, C_{2}\right)$.

Observe that in the case where $(K, H, \nu)$ is self-normalizing, Theorem 8.2 provides an alternative proof of the fact that if $\left(\theta, \theta^{\prime}\right)$ belongs to $(K, H, \nu)$ and is invariant in $(G, N, C)$, which lies over $(K, H, \nu)$, then the question of whether or not $[\theta \bullet \gamma]_{G / K C}$ is equal to $\left[\theta^{\prime} \cdot \gamma\right]_{N / H C}$ is independent of the particular $G$-invariant character $\gamma \in \operatorname{Irr}(C \mid \nu)$. In fact, by Theorem 8.2 , the ratio of these two cohomology elements is constant, independent of $\gamma$.

\section{More related characters.}

We begin with a general group-theoretic lemma.

(9.1) LEMMA. Let $G=K R$, where $K \triangleleft G$ and $K \cap R=1$. Suppose that $I \triangleleft R$ is a subgroup such that the natural map $I \rightarrow \operatorname{Aut}(K)$ (defined by conjugation in $G$ ) is an isomorphism from $I$ onto the group of inner automorphisms of $K$. For each element $k \in K$, let $k^{*} \in I$ be the element that induces the inner automorphism of $K$ induced by $k$. Let $D=\mathbf{C}_{K I}(K)$ and write $Z=D \cap K=\mathbf{Z}(K)$. The following then hold.

(a) $D \triangleleft G$.

(b) Write $\sigma(k)=k^{-1} k^{*}$ for $k \in K$. Then $\sigma$ is an $R$-isomorphism from $K$ onto $D$, and $\sigma(z)=z^{-1}$ for all $z \in Z$.

(c) $\mathbf{C}_{G}(K)=D \times \mathbf{C}_{R}(K)$.

Proof. Since $I \triangleleft R$, we see that $R$ normalizes $D$. Also, $K$ centralizes $D$, and so $D \triangleleft K R=G$, proving (a).

Since $k$ and $k^{*}$ induce the same automorphism of $K$, we see that $\sigma(k)=k^{-1} k^{*}$ centralizes $K$, and thus $\sigma$ maps $K$ into $\mathbf{C}_{K I}(K)=D$. Note that if $x, y \in K$, then $x^{*} y^{*}$ induces the same automorphism on $K$ as $x y$, and thus since $x^{*} y^{*} \in I$, we have $x^{*} y^{*}=(x y)^{*}$. Because $\sigma(x)$ centralizes $K$, we have

$$
\sigma(x) \sigma(y)=\sigma(x)\left(y^{-1} y^{*}\right)=y^{-1} \sigma(x) y^{*}=y^{-1} x^{-1} x^{*} y^{*}=(x y)^{-1}(x y)^{*}=\sigma(x y),
$$

and so $\sigma$ is a homomorphism from $K$ into $D$.

To show that $\sigma$ maps onto $D$, let $d \in D$. As $d \in K I$, we can write $d=k^{-1} r$, where $k \in K$ and $r \in I$. But $d$ centralizes $K$, and thus $k$ and $r$ induce the same automorphism of $K$ and we have $r=k^{*}$. Then $d=\sigma(k)$ and $\sigma(K)=D$, as required. 
Next, to check that $\sigma$ is injective, suppose that $x \in \operatorname{ker}(\sigma)$. Then $x=x^{*} \in K \cap I=1$, and so $\sigma$ is an isomorphism from $K$ to $D$. If $z \in Z$, then $z^{*}=1$, and so $\sigma(z)=z^{-1}$, as wanted.

To complete the proof of (b), we must show that $\sigma\left(k^{r}\right)=\sigma(k)^{r}$ for all $k \in K$ and $r \in R$. We show first that $\left(k^{r}\right)^{*}=\left(k^{*}\right)^{r}$. Certainly, $\left(k^{*}\right)^{r} \in I$ since $I \triangleleft R$, and so it suffices to show that $\left(k^{*}\right)^{r}$ and $k^{r}$ induce the same automorphism on $K$. Every element of $K$ has the form $x^{r}$ for some element $x \in K$, and we compute

$$
\left(x^{r}\right)^{k^{r}}=\left(x^{k}\right)^{r}=\left(x^{k^{*}}\right)^{r}=\left(x^{r}\right)^{\left(k^{*}\right)^{r}}
$$

as required. We thus have

$$
\sigma\left(k^{r}\right)=\left(k^{r}\right)^{-1}\left(k^{r}\right)^{*}=\left(k^{-1}\right)^{r}\left(k^{*}\right)^{r}=\left(k^{-1} k^{*}\right)^{r}=\sigma(k)^{r},
$$

and (b) is proved.

We observe next that $D \cap R=1$. This follows since if $k^{-1} k^{*} \in R$ for some element $k \in K$, then because $k^{*} \in I \subseteq R$, we have $k \in R$. Thus $k \in K \cap R=1$, and hence $k^{-1} k^{*}=1$.

Now write $M=\mathbf{C}_{R}(K)$ and note that $M \triangleleft G$ and $M \cap D=1$. We have $D M \subseteq \mathbf{C}_{G}(K)$, and so to prove (c) it suffices to show that we have equality here. Let $c \in \mathbf{C}_{G}(K)$ and write $c=k^{-1} r$ with $k \in K$ and $r \in R$. Since $c$ acts trivially on $K$, we see that $k$ and $r$ induce the same automorphism of $K$, and thus $k^{*}$ and $r$ induce the same automorphism. We have $\left(k^{*}\right)^{-1} r \in \mathbf{C}_{R}(K)=M$, and hence $c=\left(k^{-1} k^{*}\right)\left(\left(k^{*}\right)^{-1} r\right) \in D M$. This completes the proof.

(9.2) COROLLARY. Assume the situation of Lemma 9.1. Let $\lambda \in \operatorname{Irr}(Z)$ and $\varphi \in$ $\operatorname{Irr}(K \mid \lambda)$, and let $D \subseteq E \subseteq \mathbf{C}_{G}(K)$, where $E \triangleleft G$. Then there exists a character $\gamma \in \operatorname{Irr}(E \mid \lambda)$ such that the stabilizer $R_{\varphi}$ of $\varphi$ in $R$ also stabilizes $\gamma$.

Proof. Since $\mathbf{C}_{G}(K)=D \times \mathbf{C}_{R}(K)$, we see that we can write $E=D \times U$, where $U=E \cap \mathbf{C}_{R}(K) \triangleleft R$. If we can find $\tau \in \operatorname{Irr}(D \mid \lambda)$ such that $\tau$ is stabilized by $R_{\varphi}$, then the character $\gamma=\tau \times 1_{U}$ lies in $\operatorname{Irr}(E \mid \lambda)$ and is stabilized by $R_{\varphi}$, as required.

We have an $R$-isomorphism $\sigma: K \rightarrow D$, where $\sigma$ inverts $Z$, and thus the image under $\sigma$ of the character $\varphi$ lies in $\operatorname{Irr}\left(D \mid \lambda^{-1}\right)$ and is stabilized by $R_{\varphi}$. We can thus take $\tau$ to be the complex conjugate of this character.

As in the previous section, let $(K, H, \nu)$ be a self-normalizing admissible triple, and let $Z$ be the domain of the linear character $\nu$. Then $Z \subseteq H \subseteq K$ and $Z \subseteq \mathbf{Z}(K)$, and also $H=\mathbf{N}_{K}(H)$. (Note that the containment $Z \subseteq H$ follows automatically in this self-normalizing situation, and so it need not be assumed explicitly.)

Now let $\left(\theta, \theta^{\prime}\right)$ belong to $(K, H, \nu)$, where $(K, H, \nu)$ is strong. Suppose that $(G, N, C)$ lies over $(K, H, \nu)$ and that it is a relatedness candidate for $\left(\theta, \theta^{\prime}\right)$. (Recall that this means that $\left(\theta, \theta^{\prime}\right)$ is invariant in $(G, N, C)$ and that there exists at least one $G$-invariant character in $\operatorname{Irr}(C \mid \nu)$.) We seek conditions sufficient to guarantee that $\theta$ and $\theta^{\prime}$ are related in $(G, N, C)$. (In other words, we want the cohomology elements $[\theta \bullet \gamma]_{G / K C}$ and $\left[\theta^{\prime} \bullet \gamma\right]_{N / H C}$ to be equal for some, and hence for every, $G$-invariant character $\gamma \in \operatorname{Irr}(C \mid \nu)$.) 
We need one more definition. Suppose that $(G, N, C)$ lies over a self-normalizing admissible triple $(K, H, \nu)$, and recall that $C \subseteq \mathbf{C}_{G}(K)$. We say that $(G, N, C)$ is faithful over $(K, H, \nu)$ if $C=\mathbf{C}_{G}(K)$. Note that $Z=K \cap C$, and so if $(G, N, C)$ is faithful over $(K, H, \nu)$, then $Z=\mathbf{Z}(K)$, and the triple $(K, H, \nu)$ is strong. (Recall that we defined a strong triple to be a self-normalizing triple for which $Z=\mathbf{Z}(K)$.)

Suppose that $(G, N, C)$ lies over the strong triple $(K, H, \nu)$, but do not assume that it is faithful. Let $U=\mathbf{C}_{G}(K)$, so that $U \supseteq C$. Now $U \subseteq \mathbf{N}_{G}(H)=N$ and $U \cap K=\mathbf{Z}(K)=Z$, and thus $(G, N, U)$ lies over $(K, H, \nu)$. In fact, we see that $(G, N, U)$ is faithful over $(K, H, \nu)$.

(9.3) THEOREM. Let $\left(\theta, \theta^{\prime}\right)$ belong to the strong triple $(K, H, \nu)$. Assume that $\theta$ and $\theta^{\prime}$ are related in every faithful relatedness candidate lying over $(K, H, \nu)$. Then $\theta$ and $\theta^{\prime}$ are related in every relatedness candidate lying over $(K, H, \nu)$.

If $(G, N, C)$ is a relatedness candidate for $\left(\theta, \theta^{\prime}\right)$ in the situation of Theorem 9.3, let $U=\mathbf{C}_{G}(K)$. Then $(G, N, U)$ lies faithfully over $(K, H, \nu)$ and of course, $\left(\theta, \theta^{\prime}\right)$ is invariant in $(G, N, U)$. If $(G, N, U)$ is a relatedness candidate for $\left(\theta, \theta^{\prime}\right)$, then by hypothesis, $\theta$ and $\theta^{\prime}$ are related in $(G, N, U)$, and thus they are also related in $(G, N, C)$ by Theorem 7.1, the Going Down Theorem. This does not, however, prove Theorem 9.3 since in general, $(G, N, U)$ may not be a relatedness candidate for $\theta$ and $\theta^{\prime}$. The problem, of course, is that $\operatorname{Irr}(U \mid \nu)$ may not contain any $G$-invariant characters. We will use the following lemma to overcome this difficulty.

(9.4) LEMMA. Suppose that $\left(\theta, \theta^{\prime}\right)$ belongs to the strong triple $(K, H, \nu)$ and that $(G, N, C)$ is a relatedness candidate for $\left(\theta, \theta^{\prime}\right)$. Then there exist two new relatedness candidates $\left(G^{*}, N^{*}, F\right)$ and $\left(G^{*}, N^{*}, E\right)$ for $\left(\theta, \theta^{\prime}\right)$ such that the following hold.

(a) $\left(G^{*}, N^{*}, F\right)$ is faithful over $(K, H, \nu)$.

(b) $\mathbf{Z}(K) \subseteq E \subseteq F$.

(c) $\left(G^{*}, N^{*}, E\right)$ is equivalent to $(G, N, C)$.

(d) $G^{*}$ splits over $K$ and a complement contains a normal subgroup that acts faithfully on $K$ and induces the group of inner automorphisms of $K$.

Before we prove Lemma 9.4, we deduce Theorem 9.3 from it.

Proof of Theorem 9.3 Let $\left(G^{*}, N^{*}, F\right)$ and $\left(G^{*}, N^{*}, E\right)$ be as in Lemma 9.4. Since $\left(G^{*}, N^{*}, F\right)$ is a faithful relatedness candidate, it follows by hypothesis that $\theta$ and $\theta^{\prime}$ are related in $\left(G^{*}, N^{*}, F\right)$. By Theorem 7.1, the Going Down Theorem, we conclude that $\theta$ and $\theta^{\prime}$ are also related in $\left(G^{*}, N^{*}, E\right)$. Finally, since $\left(G^{*}, N^{*}, E\right)$ is equivalent to $(G, N, C)$, we conclude by Corollary 8.3 that $\theta$ and $\theta^{\prime}$ are related in $(G, N, C)$.

Proof of Lemma 9.4. Let $Z=\mathbf{Z}(K) \triangleleft G$ and write $\bar{G}=G / Z$. Then $\bar{G}$ acts on $K$, and we let $G^{*}=K \rtimes \bar{G}$ be the semidirect product. (As is customary, we identify $K$ and $\bar{G}$ with their images in the semidirect product and write $G^{*}=K \bar{G}$.) If $X$ is any subgroup of $G$, then $\bar{X} \subseteq \bar{G}$, and we view $\bar{X}$ as a subgroup of $G^{*}$ contained in the complement $\bar{G}$ of $K$.

Note that the complement $\bar{G}$ contains $\bar{K}$ as a normal subgroup, and $\bar{K}$ acts faithfully on $K$ and induces the group of inner automorphisms on $K$, as is required for (d). Also, this 
enables us to apply Lemma 9.1 with $I=\bar{K}$, and as in that lemma, we let $D=\mathbf{C}_{K I}(K)$. By Lemma 9.1(b), we know that $D=\left\{k^{-1} \bar{k} \mid k \in K\right\}$.

Let $U=\mathbf{C}_{G}(K)$ and note that $\bar{U}=\mathbf{C}_{\bar{G}}(K)$. Let $F=\mathbf{C}_{G^{*}}(K)$. By Lemma 9.1(c), we can write $F=D \times \bar{U}$, and we have $Z \subseteq D \triangleleft G^{*}$ by 9.1 (a). Also $C \subseteq U$, and so $\bar{C} \subseteq \bar{U}$ and we let $E=D \times \bar{C} \subseteq F$ and $N^{*}=\mathbf{N}_{G^{*}}(H)$. We will show that $\left(G^{*}, N^{*}, E\right)$ and $\left(G^{*}, N^{*}, F\right)$ lie over $(K, H, \nu)$ and that they are relatedness candidates for $\theta$ and $\theta^{\prime}$. Also, we will show that $\left(G^{*}, N^{*}, E\right)$ is equivalent to $(G, N, C)$.

We construct an isomorphism $\eta: G^{*} / E \rightarrow G / C$ such that $\eta$ carries $E k$ to $C k$ for all elements $k \in K$, and such that for each coset $X$ of $E$ in $G^{*}$, the elements of $X$ and the elements of $\eta(X)$ induce the same automorphism of $K$.

Let $\alpha$ be the the restriction to $K$ of the canonical homomorphism $G \rightarrow G / C$, so that $\alpha(k)=C k$ for $k \in K$. Also, define the homomorphism $\beta: \bar{G} \rightarrow G / C$ by $\beta(\bar{g})=C g$. (This is well-defined since $Z \subseteq C$.) For $k \in K$ and $\bar{g} \in \bar{G}$, we see that

$$
\alpha\left(k^{\bar{g}}\right)=\alpha\left(k^{g}\right)=(C k)^{C g}=\alpha(k)^{\beta(\bar{g})} .
$$

It follows that there is a homomorphism $\xi$ from the semidirect product $G^{*}=K \rtimes \bar{G}$ into $G / C$ defined by $\xi(k \bar{g})=\alpha(k) \beta(\bar{g})$. In particular, we see that $\xi(k \bar{g})=(C k)(C g)=C(k g)$.

Now $\xi$ maps $G^{*}$ onto $G / C$, and we compute $\operatorname{ker}(\xi)$. Observe that $k^{-1} \bar{g} \in \operatorname{ker}(\xi)$ if and only if $k^{-1} g \in C$. In particular, if this happens, we have

$$
k^{-1} \bar{g}=\left(k^{-1} \bar{k}\right)\left(\overline{k^{-1} g}\right) \in D \bar{C}=E
$$

and so $\operatorname{ker}(\xi) \subseteq E$. Conversely, every element of $E=D \bar{C}$ has the form $e=\left(k^{-1} \bar{k}\right)(\bar{c})$ with $k \in K$ and $c \in C$. Then $e=k^{-1} \overline{k c}$, and since $\left(k^{-1}\right)(k c) \in C$, it follows that $e \in \operatorname{ker}(\xi)$. Thus $\operatorname{ker}(\xi)=E$ and $\xi$ induces an isomorphism $\eta: G^{*} / E \rightarrow G / Z$, as wanted.

If $x=k \bar{g} \in G^{*}$, then $\eta(E x)=\xi(x)=C k g$, and the elements of this coset of $C$ induce the same automorphism of $K$ as does $x$, as required. Finally, we observe that if $k \in K$, then $\eta(E k)=\xi(k)=C k$, and thus $\eta$ has the desired properties.

Now $\mathbf{Z}(K)=Z \subseteq D \subseteq E \subseteq F=\mathbf{C}_{G^{*}}(K)$, and so $E \cap K=Z=F \cap K$. In order to show that both $\left(G^{*}, N^{*}, E\right)$ and $\left(G^{*}, N^{*}, F\right)$ lie over $(K, H, \nu)$, there are just two more things we need to check: that $K \cap N^{*}=H$ and that $K N^{*}=G^{*}$. The first of these is clear since $H=\mathbf{N}_{K}(H)$. To prove the second statement, recall that the elements of a coset $X$ of $E$ in $G^{*}$ induce the same automorphism of $K$ as do the elements of the coset $\eta(X)$. Since $N=\mathbf{N}_{G}(H)$ and $N^{*}=\mathbf{N}_{G^{*}}(H)$, it follows that $\eta\left(N^{*} / E\right)=N / C$. Also, we know that $\eta(K E / E)=K C / C$, and since $(N / C)(K C / C)=G / C$, it follows from the fact that $\eta$ is an isomorphism that $\left(N^{*} / E\right)(K E / E)=G^{*} / E$. Thus $N^{*} K=G^{*}$, as needed.

Since $(G, N, C)$ and $\left(G^{*}, N^{*}, E\right)$ are equivalent, it follows from the fact that $\theta$ and $\theta^{\prime}$ are $N$-invariant that they are also $N^{*}$-invariant. To complete the proof, all that remains is to show that $\operatorname{Irr}(F \mid \nu)$ and $\operatorname{Irr}(E \mid \nu)$ contain $G^{*}$-invariant characters. In fact, it suffices to show that each of these sets contains a $\bar{G}$-invariant character because $K$ centralizes $E$ and $F$. But $K$ certainly contains a $\bar{G}$-invariant character, namely $\theta$, and thus we are done by Corollary 9.2 . 


\section{Good simples and perfect groups.}

Finally, we are ready to define what it means for a nonabelian simple group of order divisible by $p$ to be "good" for $p$. We give that definition here, and then in the following three sections, we prove that simple groups that are good for $p$ must lie in the class $\mathcal{X}$, which was defined in Section 1. In particular, this will prove Theorem E.

Fix a prime $p$. Given a nonabelian simple group $X$ of order divisible by $p$, we consider perfect groups $S$ such that $S / Z \cong X$, where $Z=\mathbf{Z}(S)$ is cyclic with order not divisible by $p$. We are about to state several conditions that we want the group $S$ to satisfy, and by definition, the simple group $X$ is good for the prime $p$ if all of these conditions hold for every choice of $S$. (Note that up to isomorphism, there are just finitely many such groups $S$ for each simple group, and thus in principle, it is a finite problem to decide whether or not the given simple group $X$ is good for $p$.)

Fix a faithful linear character $\nu$ of $Z$ and a Sylow $p$-subgroup $Q$ of $S$. (Since all possibilities for $\nu$ are Galois-conjugate and all possibilities for $Q$ are conjugate in $S$, it will be clear that the particular choice of the faithful character $\nu$ and the Sylow subgroup $Q$ are irrelevant to the conditions we will state.)

Let $A$ be the subgroup of $\operatorname{Aut}(S)$ consisting of all automorphisms that act trivially on $Z$ and stabilize $Q$. We require that there exists a subgroup $T$ of $S$ such that

(1) $T$ is stabilized by $A$.

(2) $\mathbf{N}_{S}(Q) \subseteq T<S$.

(3) There is a bijection $\theta \mapsto \theta^{*}$ from $\operatorname{Irr}_{p^{\prime}}(S \mid \nu)$ to $\operatorname{Irr}_{p^{\prime}}(T \mid \nu)$.

Note that Condition (3) makes sense since $Z \subseteq \mathbf{N}_{S}(Q) \subseteq T$ by (2). Our remaining conditions refer to this subgroup $T$ and the bijection ()$^{*}$.

Observe that $A$ fixes $\nu$, and so $A$ acts on the sets $\operatorname{Irr}_{p^{\prime}}(S \mid \nu)$ and $\operatorname{Irr}_{p^{\prime}}(T \mid \nu)$. We require that

(4) The map ( )* is $A$-equivariant.

This means that ( $)^{*}$ defines a permutation isomorphism between the actions of $A$ on $\operatorname{Irr}_{p^{\prime}}(S \mid \nu)$ and on $\operatorname{Irr}_{p^{\prime}}(T \mid \nu)$. In other words, $\left(\theta^{a}\right)^{*}=\left(\theta^{*}\right)^{a}$ for all characters $\theta \in \operatorname{Irr}_{p^{\prime}}(S \mid \nu)$ and all automorphisms $a \in A$.

Before we state our remaining conditions, we make the following observation.

(10.1) LEMMA. Assume the above notation and that Conditions (1), (2), (3) and (4) are satisfied. Suppose that $S$ is normal in some group $G$ and that $Z \subseteq \mathbf{Z}(G)$. Let $N=\mathbf{N}_{G}(T)$. Then $G=S N$ and $T=S \cap N$. Also, the character bijection ( )* is $N$-equivariant.

Proof. Let $M=\mathbf{N}_{G}(Q)$ and observe that conjugation by an element of $M$ induces an automorphism of $S$ that lies in the group $A$. By Condition (1), therefore, $M \subseteq \mathbf{N}_{G}(T)=N$, and by Condition (4), the map ( $)^{*}$ is $M$-equivariant. Since $G=S M$ by the Frattini argument, we have $G=S N$, as required. Also, $N=T M$ by the Frattini argument, and since $T$ acts trivially on characters of $S$ and on characters of $T$, it follows that ( )* is $N$-equivariant. Finally, by Dedekind's lemma, $S \cap N=T(S \cap M)=T$, since $S \cap M \subseteq T$ by Condition (2).

Returning now to our definition of "good", we fix $\theta \in \operatorname{Irr}_{p^{\prime}}(S \mid \nu)$. We require that the remainder of our conditions hold for every choice of $\theta$. Let $B=A_{\theta}$, the stabilizer of $\theta$ in 
$A$, and note that by Condition (4), $B$ is also the stabilizer of $\theta^{*}$ in $A$.

We require next that $S$ can be normally embedded in some group $G$ (which may depend on $\theta$ ) such that $G$ has several additional properties. The first of these is the following.

(5) $Z \subseteq \mathbf{Z}(G)$ and $B$ is exactly the group of automorphisms of $S$ induced by the conjugation action of the subgroup $M=\mathbf{N}_{G}(Q)$ on $S$.

In particular, since $\theta$ is $B$-invariant, it is $M$-invariant, and hence it is $G$-invariant because $G=S M$ by the Frattini argument. We mention that the existence of a group $G$ satisfying (5) is automatic. It is not hard to see, for example, that the semidirect product $S \rtimes B$ has the desired properties.

We continue to assume that $\theta$ is given, that $B=A_{\theta}$, and that we have chosen and fixed a group $G$ such that $S \triangleleft G$ and Condition (5) holds. The remaining conditions will be stated in terms of $G$ and $\theta$. We require that

(6) The subgroup $C=\mathbf{C}_{G}(S)$ is abelian.

(7) The set $\operatorname{Irr}(C \mid \nu)$ contains a $G$-invariant character $\gamma$.

Our final requirement is that certain cohomology elements are equal. To state this condition precisely, let $N=\mathbf{N}_{G}(T)$ and observe that $C \subseteq N$. By Lemma 10.1, we have $S N=G$ and $S \cap N=T$, and so there is a natural isomorphism $G / S C \cong N / T C$, and this enables us to identify the Schur multipliers of these groups. Also, $\theta$ is $G$-invariant, and thus $\theta^{*}$ is $N$-invariant by Lemma 10.1. Then $\theta \cdot \gamma \in \operatorname{Irr}(S C)$ is $G$-invariant and $\theta^{*} \cdot \gamma \in \operatorname{Irr}(T C)$ is $N$-invariant, where $\gamma$ is as in Condition (7). We require that

$$
[\theta \cdot \gamma]_{G / S C}=\left[\theta^{*} \cdot \gamma\right]_{N / T C}
$$

We can restate most of this in the language that we established in the foregoing sections. We have $Z=\mathbf{Z}(S) \subseteq T \subseteq S$ and $\nu \in \operatorname{Irr}(Z)$, and thus $(S, T, \nu)$ is an admissible triple. Also, $T=S \cap N=\mathbf{N}_{S}(T)$, and so the triple $(S, T, \nu)$ is self-normalizing, and in fact, it is strong since $Z$ is the full center of $S$. Also, $G=S N$ and $S \cap N=T$, and we have $C \cap S=\mathbf{Z}(S)=Z$, and thus $(G, N, C)$ lies over $(S, T, \nu)$. In fact, $(G, N, C)$ is faithful over $(S, T, \nu)$ since $C=\mathbf{C}_{G}(S)$. Furthermore, $\left(\theta, \theta^{*}\right)$ belongs to $(S, T, \nu)$ and is invariant in $(G, N, C)$. Finally, $(G, N, C)$ is a relatedness candidate for $\left(\theta, \theta^{*}\right)$ because of Condition ( 7 ), and $\theta$ and $\theta^{*}$ are related in $(G, N, C)$ by Condition (8).

These conditions guarantee that a somewhat stronger version of Condition (8) holds.

(10.2) LEMMA. Let $S \subseteq G_{0} \subseteq G$ in the above situation, and define $N_{0}=N \cap G_{0}$ and $C_{0}=C \cap G_{0}$. Then $\left(G_{0}, N_{0}, C_{0}\right)$ lies over $(S, T, \nu)$ and $\theta$ and $\theta^{*}$ are related in $\left(G_{0}, N_{0}, C_{0}\right)$.

Proof. It is clear that $\left(G_{0}, N_{0}, C_{0}\right)$ lies over $(S, T, \nu)$ and that $\left(\theta, \theta^{*}\right)$ is invariant in $\left(G_{0}, N_{0}, C_{0}\right)$. Also, since $C$ is abelian by Condition (6), we know that the $G$-invariant character $\gamma$ of Condition (7) is linear, and thus its restriction $\gamma_{0}=\gamma_{C_{0}}$ is irreducible, and of course, it is $G_{0}$-invariant. In other words, $\left(G_{0}, N_{0}, C_{0}\right)$ is a relatedness candidate for $\left(\theta, \theta^{*}\right)$.

Under the identification of $G / S C$ with $N / T C$, the subgroups $G_{0} C / S C$ and $N_{0} C / T C$ correspond. Since $[\theta \cdot \gamma]_{G / S C}=\left[\theta^{*} \cdot \gamma\right]_{N / T C}$, it is immediate (by restriction of factor sets) that $[\theta \cdot \gamma]_{G_{0} C / S C}=\left[\theta^{*} \cdot \gamma\right]_{N_{0} C / T C}$. Observe that $G_{0} C / S C$ is naturally isomorphic to $G_{0} / S C_{0}$ and that the character $\theta \bullet \gamma \in \operatorname{Irr}(S C)$ restricts to $\theta \bullet \gamma_{0} \in \operatorname{Irr}\left(S C_{0}\right)$. Since this 
restriction is irreducible, it follows by Lemma 3.6 that $[\theta \bullet \gamma]_{G_{0} C / S C}=\left[\theta \bullet \gamma_{0}\right]_{G_{0} / S C_{0}}$ and similarly, $\left[\theta^{*} \cdot \gamma\right]_{N_{0} C / T C}=\left[\theta^{*} \cdot \gamma_{0}\right]_{N_{0} / T C_{0}}$. Combining our equalities, we get $\left[\theta \bullet \gamma_{0}\right]_{G_{0} / S C_{0}}=$ $\left[\theta^{*} \cdot \gamma_{0}\right]_{N_{0} / T C_{0}}$, and thus $\theta$ and $\theta^{*}$ are related in $\left(G_{0}, N_{0}, C_{0}\right)$, as claimed.

We close this section with the following observation.

(10.3) LEMMA. Let $X$ be a simple group that has trivial Schur multiplier and trivial outer automorphism group, and assume that the McKay conjecture holds for $X$ with respect to some prime divisor $p$ of $|X|$. Then $X$ is good for $p$.

Proof. Since the Schur multiplier of $X$ is trivial, it follows that the group $Z$ in the statement of our conditions is trivial, and thus $S=X$ and $\nu$ is the principal character of $Z$. Also, since all automorphisms of $X$ are inner, we see that $A$ is exactly the group of automorphisms of $X$ induced by conjuation by elements of $\mathbf{N}_{X}(Q)$, where $Q \in \operatorname{Syl}_{p}(X)$. Take $T=\mathbf{N}_{X}(Q)$, so that Conditions (1) and (2) hold. Condition (3) is exactly the assumption that $X$ satisfies the McKay conjecture for the prime $p$. Also, since $A$ acts trivially on $\operatorname{Irr}(X)$ and $\operatorname{Irr}(T)$, Condition (4) holds.

Now fix $\theta \in \operatorname{Irr}_{p^{\prime}}(X)$, and note that $B=A_{\theta}=A$. We can take $G=X$, and we observe that Condition (5) holds. The group $C$ of Condition (6) is trivial, and hence (6) holds. Also (7) holds, with $\gamma$ being the principal character of the trivial group $C$. Finally, the groups $G / S C$ and $N / T C$ of Condition (8) are trivial, and thus the cohomology elements of (8) are both trivial, and hence they are equal.

\section{Correspondence subgroups.}

Suppose $X$ is a simple group that is good for the prime $p$. As in the previous section, let $S$ be perfect with $S / Z \cong X$, where $Z=\mathbf{Z}(S)$ is a cyclic $p^{\prime}$-group. Also, fix $Q \in \operatorname{Syl}_{p}(S)$ and $\nu \in \operatorname{Irr}(Z)$, where $\nu$ is faithful. Since we are assuming that $X$ is good for $p$, we know (by definition) that there exists at least one subgroup $T$ of $S$ and a corresponding bijection $\theta \mapsto \theta^{*}$ from $\operatorname{Irr}_{p^{\prime}}(S \mid \nu)$ to $\operatorname{Irr}_{p^{\prime}}(T \mid \nu)$ with the properties we enumerated. We refer to $T$ as a correspondence subgroup for $S, Q$ and $\nu$, and we call the map ( )* the associated correspondence.

We do not claim that the correspondence subgroup $T$ is uniquely determined by $S$, $Q$ and $\nu$, nor do we claim that the associated correspondence ( $)^{*}$ is unique. To remedy this potential ambiguity, consider the equivalence relation on triples $(S, Q, \nu)$ defined by declaring $\left(S_{1}, Q_{1}, \nu_{1}\right)$ to be equivalent to $\left(S_{2}, Q_{2}, \nu_{2}\right)$ if there is a group isomorphism $\tau$ : $S_{1} \rightarrow S_{2}$ such that $\tau\left(Q_{1}\right)=Q_{2}$ and (with the obvious meaning) $\tau\left(\nu_{1}\right)=\nu_{2}$. Choose a representative in each equivalence class of triples, and for each such representative, choose and fix one correspondence subgroup and its associated correspondence.

Using the isomorphisms among the members of an equivalence class, we can now define correspondence subgroups and associated correspondences unambiguously for all members of the class. To see that this can be done, we must show that if $\tau$ and $\sigma$ are two isomorphisms from $S_{1}$ to $S_{2}$, and both carry $Q_{1}$ to $Q_{2}$ and $\nu_{1}$ to $\nu_{2}$, then both $\tau$ and $\sigma$ carry a correspondence subgroup $T_{1} \subseteq S_{1}$ to the same subgroup $T_{2}$ of $S_{2}$. We must also show, of course, that the associated correspondences defined by $\tau$ and $\sigma$ are identical.

To check these conditions, note that $\sigma$ followed by $\tau^{-1}$ is an automorphism of $S_{1}$ that centralizes $Z_{1}$ and stabilizes $Q_{1}$, and hence it lies in the group $A_{1}$ (corresponding to the 
group that was previously called $A$ ). But then by Conditions (1) and (4), it follows that $T_{1}$ and ( )* are stabilized by this automorphism, and this establishes what we need.

We have now defined a particular correspondence subgroup $T$ and a particular correspondence ( $)^{*}$ for each choice of $S, Q$ and $\nu$. We write $T=\mathbf{T}(S, Q, \nu)$, but to avoid notational clutter, we will not attempt to indicate the dependence of the associated correspondence on the initial data. Instead, we use the generic notation ( )* in all cases. Because of the way we have selected our correspondence subgroups, we know that if $\tau: S_{1} \rightarrow S_{2}$ is an isomorphism taking $Q_{1}$ to $Q_{2}$ and $\nu_{1}$ to $\nu_{2}$, as above, then $\tau$ carries $\mathbf{T}\left(S_{1}, Q_{1}, \nu_{1}\right)$ to $\mathbf{T}\left(S_{2}, Q_{2}, \nu_{2}\right)$ and $\tau$ respects the associated correspondences.

If $T=\mathbf{T}(S, Q, \nu)$, we see that $\mathbf{N}_{S}(Q) \subseteq T$ by Condition (2) of the previous section, and thus $T$ is self-normalizing in $S$ by the Frattini argument. Since $Z=\mathbf{Z}(S)$, it follows that $(S, T, \nu)$ is a strong triple.

(11.1) THEOREM. Let $S, Q, Z$ and $\nu$ be as above, and write $T=\mathbf{T}(S, Q, \nu)$. Let $\theta \in$ $\operatorname{Irr}_{p^{\prime}}(S \mid \nu)$ and suppose that $(G, N, C)$ is a relatedness candidate for $\left(\theta, \theta^{*}\right)$ over $(S, T, \nu)$. Then $\theta$ and $\theta^{*}$ are related in $(G, N, C)$.

We stress that $G, N$ and $C$ in Theorem 11.1 are not necessarily the groups with those names that were discussed in the previous section. Here, $(G, N, C)$ is an arbitrary relatedness candidate for $\left(\theta, \theta^{*}\right)$, and in particular, we are not assuming that it is faithful or that $C$ is abelian.

Proof of Theorem 11.1. To prove that $\theta$ and $\theta^{*}$ are related in $(G, N, C)$, it suffices by Theorem 9.3 to show that $\theta$ and $\theta^{*}$ are related in every faithful relatedness candidate over $(S, T, \nu)$. It is therefore no loss to assume that $(G, N, C)$ is faithful, and so we assume that $C=\mathbf{C}_{G}(S)$.

Since $\theta$ is $N$-invariant and $G=S N$, we see that $\theta$ is $G$-invariant. Thus $\theta$ is invariant under $M=\mathbf{N}_{G}(S)$, and hence it is also invariant under the group $B_{0}$ of automorphisms of $S$ induced by conjugation via elements of $M$. Observe that $B_{0}$ is a subgroup of the full stabilizer $B$ of $\theta$ in the group of all automorphism of $S$ that stabilize $Q$ and act trivially on $Z$.

By Condition (5) of the definition of "good", $S$ is normal in a certain group that was called $G$ in the previous section, but which we call $G_{1}$ here. We know that $Z \subseteq \mathbf{Z}\left(G_{1}\right)$ and that certain additional properties hold, and in particular, the subgroup $M_{1}=\mathbf{N}_{G_{1}}(Q)$ induces the full group $B$ of automorphisms on $S$. Let $C_{1}=\mathbf{C}_{G_{1}}(S)$ and $N_{1}=\mathbf{N}_{G_{1}}(T)$, and observe that $\left(G_{1}, N_{1}, C_{1}\right)$ lies over $(S, T, \nu)$. (This follows by Lemma 10.1.)

Let $M_{0} \subseteq M_{1}$ be the subgroup consisting of all elements of $M_{1}$ that induce automorphisms of $S$ that lie in the group $B_{0}$. Since $B_{0} \subseteq B$ and $M_{1}$ induces the full group $B$ on $S$, we see that $M_{0}$ induces the group $B_{0}$ on $S$. Let $N_{0}=T M_{0}, G_{0}=S M_{0}$ and $C_{0}=C_{1} \cap G_{0}=\mathbf{C}_{G_{0}}(S)$. It is easy to check that this puts us into the situation of Lemma 10.2, and hence $\theta$ and $\theta^{*}$ are related in $\left(G_{0}, N_{0}, C_{0}\right)$.

By Corollary 8.3, it suffices to show that $(G, N, C)$ and $\left(G_{0}, N_{0}, C_{0}\right)$ are equivalent over $(S, T, \nu)$. To establish this, we need an isomorphism $\eta: G / C \rightarrow G_{0} / C_{0}$ such that

(1) $C x$ and $\eta(C x)$ induce identical automorphisms of $S$ for all $x \in G$ and

(2) $\eta(C s)=C_{0} s$ for all elements $s \in S$. 
Since $(G, N, C)$ lies faithfully over $(S, T, \nu)$, we know that $G / C$ is isomorphic to the group $U$ of automorphisms of $S$ induced by $G$. Also, since $G=S M$ by the Frattini argument and $M$ induces the group $B_{0}$ on $S$, we have $U=I B_{0}$, where $I$ is the group of inner automorphisms of $S$. Since $M_{0}$ also induces the group $B_{0}$ and $G_{0}=S M_{0}$, it follows that the group of automorphisms of $S$ induced by $G_{0}$ is also $I B_{0}=U$. Then $G / C$ and $G_{1} / C_{1}$ are both isomorphic to the same group $U$ of automorphisms of $S$, and therefore there exists an isomorphism $\eta: G / C \rightarrow G_{1} / C_{1}$ such that (1) holds.

Since the coset $C_{0} s$ is the full set of elements of $G_{0}$ that induce the inner automorphism induced by $s$, it follows that $\eta(C s)=C_{0} s$ and (2) holds also. This completes the proof.

\section{Groups that may not be perfect.}

Fix a prime $p$ and let $X$ be a simple group that is good for $p$. Suppose that $S / Z \cong X$, where $Z=\mathbf{Z}(S)$ is a cyclic $p^{\prime}$-group, and choose a faithful linear character $\nu$ of $Z$ and a Sylow subgroup $Q \in \operatorname{Syl}_{p}(S)$. In the previous section we considered the case where $S$ is perfect, and we defined a correspondence subgroup $T=\mathbf{T}(S, Q, \nu)$ and an associated character correspondence $\theta \mapsto \theta^{*}$ from $\operatorname{Irr}_{p^{\prime}}(S \mid \nu)$ onto $\operatorname{Irr}_{p^{\prime}}(T \mid \nu)$. Our goal here is to do the same thing, but without assuming that $S$ is perfect.

Given $S, Q$ and $\nu$ as above, let $U=S^{\prime}$ and write $Y=U \cap Z$. Since $S / Z$ is simple, we have $S=Z U$, and so $U=S^{\prime}=U^{\prime}$ and $U$ is perfect. Also, $Y=\mathbf{Z}(U)$ is a cyclic $p^{\prime}$ group and $U / Y \cong S / Z \cong X$. Furthermore, since $Z$ is a $p^{\prime}$-group, the index $|S: U|$ is not divisible by $p$, and thus $Q \in \operatorname{Syl}_{p}(U)$. Finally, writing $\mu=\nu_{Y}$, we see that $\mu$ is a faithful linear character of $Y$, and so the correspondence subgroup $V=\mathbf{T}(U, Q, \mu)$ is defined, and we write $T=Z V$. We can now extend our previous definitions to not-necessarily-perfect groups by writing $\mathbf{T}(S, Q, \nu)=T$. We refer to $T$ as a correspondence subgroup for $S$ (with respect to $Q$ and $\nu$ ).

Observe that

$$
U \cap T=U \cap Z V=(U \cap Z) V=Y V=V
$$

Since we know that $V<U$ by Condition (2) of Section 10, it follows that $T<S$. Also, if $S$ is normal in some group $G$, then $U \triangleleft G$ and $Z \triangleleft G$ and it follows from the equations $T=Z V$ and $V=U \cap T$ that $\mathbf{N}_{G}(T)=\mathbf{N}_{G}(V)$. In particular, $\mathbf{N}_{S}(T)=\mathbf{N}_{S}(V)=Z V=T$, where the second equality follows from the fact that $V=\mathbf{N}_{U}(V)$. We conclude that $(S, T, \nu)$ is a strong triple.

Next, we construct a bijection $\operatorname{Irr}_{p^{\prime}}(S \mid \nu) \rightarrow \operatorname{Irr}_{p^{\prime}}(T \mid \nu)$. Since $S=Z U$ is a central product and $Y=U \cap Z$, the map $\alpha \mapsto \alpha \bullet \nu$ is a degree-preserving bijection from $\operatorname{Irr}(U \mid \mu)$ to $\operatorname{Irr}(S \mid \nu)$. In particular, this map defines a bijection from $\operatorname{Irr}_{p^{\prime}}(U \mid \mu)$ to $\operatorname{Irr}_{p^{\prime}}(S \mid \nu)$. Similarly, $T=Z V$ is a central product and the map $\beta \mapsto \beta \bullet \nu$ defines a bijection from $\operatorname{Irr}_{p^{\prime}}(V \mid \mu)$ to $\operatorname{Irr}_{p^{\prime}}(T \mid \nu)$. Furthermore, since $V$ is a correspondence subgroup for $U$, we have an associated correspondence ( $)^{*}$ from $\operatorname{Irr}_{p^{\prime}}(U \mid \mu)$ to $\operatorname{Irr}_{p^{\prime}}(V \mid \mu)$. We can combine these maps to define a bijection from $\operatorname{Irr}(S \mid \nu)$ to $\operatorname{Irr}(T \mid \nu)$. (We also call this map ( )* and refer to it as the associated correspondence.) To be precise, we have $(\alpha \cdot \nu)^{*}=\alpha^{*} \cdot \nu$.

The properties of correspondence subgroups and their associated correspondences that we established in the previous section also hold in this generality. For example, if $\tau: S_{1} \rightarrow$ $S_{2}$ is an isomorphism that carries Sylow $p$-subgroup $Q_{1}$ to Sylow $p$-subgroup $Q_{2}$ and linear 
character $\nu_{1}$ of $Z_{1}=\mathbf{Z}\left(S_{1}\right)$ to linear character $\nu_{2}$ of $Z_{2}=\mathbf{Z}\left(S_{2}\right)$, then it should be clear that $\tau$ maps $\mathbf{T}\left(S_{1}, Q_{1}, \nu_{1}\right)$ to $\mathbf{T}\left(S_{2}, Q_{2}, \nu_{2}\right)$ and $\tau$ respects the relevant character bijections.

(12.1) THEOREM. Let $S, Q, Z$ and $\nu$ be as before, where $S$ is not necessarily perfect, and write $T=\mathbf{T}(S, Q, \nu)$. Let $\theta \in \operatorname{Irr}_{p^{\prime}}(S \mid \nu)$ and suppose that $(G, N, C)$ is a relatedness candidate for $\left(\theta, \theta^{*}\right)$ over $(S, T, \nu)$. Then $\theta$ and $\theta^{*}$ are related in $(G, N, C)$.

Proof. As before, we let $U=S^{\prime}, Y=U \cap Z, \mu=\nu_{Y}$ and $V=\mathbf{T}(U, Q, \mu)$, so that we have $T=Z V$, and $V=T \cap U$, and thus $N=\mathbf{N}_{G}(V)$. We argue first that $(G, N, C)$ lies over $(U, V, \mu)$. We have $G=N S=N Z U=N U$, and also, $V=T \cap U=(N \cap S) \cap U=N \cap U$. Of course, $C$ centralizes $U$ and $Y=Z \cap U=(C \cap S) \cap U=C \cap U$, as required.

Write $\theta=\alpha \cdot \nu$ and recall that $\theta^{*}=\alpha^{*} \cdot \nu$, where $\left(\alpha, \alpha^{*}\right)$ belongs to $(U, V, \mu)$. Also, observe that $\alpha$ and $\alpha^{*}$ are the restrictions of $\theta$ and $\theta^{*}$ to $U$ and $V$, and thus $\left(\alpha, \alpha^{*}\right)$ is invariant in $(G, N, C)$. Since by assumption, $(G, N, C)$ is a relatedness candidate for $\left(\theta, \theta^{*}\right)$, we know that there is a $G$-invariant character $\gamma \in \operatorname{Irr}(C \mid \nu) \subseteq \operatorname{Irr}(C \mid \mu)$, and it follows that $(G, N, C)$ is a relatedness candidate for $\left(\alpha, \alpha^{*}\right)$.

By Theorem 11.1, the characters $\alpha$ and $\alpha^{*}$ are related in $(G, N, C)$, and so if we identify $G / U C=N / V C$, we can write $[\alpha \cdot \gamma]_{G / U C}=\left[\alpha^{*} \cdot \gamma\right]_{N / V C}$.

Now $S C=Z U C=U C$ and $T C=Z V C=V C$, and since $\gamma$ lies over $\nu$, we have $\theta \cdot \gamma=\alpha \cdot \gamma$ and similarly, $\theta^{*} \cdot \gamma=\alpha^{*} \cdot \gamma$. The result now follows.

\section{Semisimple quotients.}

Let $K$ be a finite group and let $Z=\mathbf{Z}(K)$, where $Z$ is cyclic of order not divisible by $p$. In the previous two sections we considered the case where $K / Z$ is a simple group that is good for $p$, but now we relax that condition and assume instead that $K / Z$ is a direct product of simple groups, each of which is good for $p$. As before, we let $\nu$ be a faithful linear character of $Z$, and we fix a Sylow subgroup $Q \in \operatorname{Syl}_{p}(K)$.

The main result of this section is the following, which includes Theorem $\mathrm{E}$ of the introduction. (The subgroup $N$ of Theorem 13.1 is the subgroup whose existence was asserted in Theorem E, but which was called $M$ in that theorem.)

(13.1) THEOREM. Let $K, Z, Q$ and $\nu$ be as above. Then there exists a subgroup $H$ of $K$ and a character correspondence ( $)^{*}$ from $\operatorname{Irr}_{p^{\prime}}(K \mid \nu)$ to $\operatorname{Irr}_{p^{\prime}}(H \mid \nu)$ such that

(a) $\mathbf{N}_{K}(Q) \subseteq H<K$.

Now suppose that $K$ is normal in some group $G$ with $Z \subseteq \mathbf{Z}(G)$, and write $N=\mathbf{N}_{G}(H)$. The following then hold.

(b) $K N=G$ and $K \cap N=H$.

(c) $\mathbf{N}_{G}(Q) \subseteq N<G$.

(d) The bijection ( )* is $N$-equivariant.

(e) $\left|\operatorname{Irr}_{p^{\prime}}(G \mid \theta)\right|=\left|\operatorname{Irr}_{p^{\prime}}\left(N \mid \theta^{*}\right)\right|$ for all characters $\theta \in \operatorname{Irr}_{p^{\prime}}(K \mid \nu)$.

Recall that in the statement of Theorem E, we assumed Hypothesis D, which was that $K / \mathbf{Z}(G)$ is the direct product of simple groups all of which are isomorphic to some group $X$, where $X$ is good for $p$. The hypothesis of Theorem 13.1 is more general in two respects: we do not require $Z$ to be the full center of $G$ and we do not require that the simple direct summands of $K / Z$ are all isomorphic. 
Since Theorem 13.1 includes Theorem E, a consequence is that every simple group that is good for $p$ lies in the class $\mathcal{X}$, which was defined in Section 1. It follows by Theorem 2.1 that the relative McKay conjecture (Conjecture C) holds for $L \triangleleft G$ if all nonabelian simple groups involved in $G / L$ and having order divisible by $p$ are good for $p$. In particular, this proves Theorem B.

We now begin work toward a proof of Theorem 13.1. Since $K / Z$ is a direct product of good (for $p$ ) simple groups and these, by definition, are nonabelian, we know that $K / Z$ is the direct product of the groups $S / Z$, where $S$ runs over the collection $\mathcal{S}$ consisting of all subgroups of $K$ such that $Z \subseteq S \triangleleft K$ and $S / Z$ is simple. Note that $Z=\mathbf{Z}(S)$ for all groups $S \in \mathcal{S}$. Also, as we saw in Section 5 , the distinct members of $\mathcal{S}$ centralize each other, and so $K$ is the central product of the members of $\mathcal{S}$. Let $e=|\mathcal{S}|$.

If $S \in \mathcal{S}$, then $Q \cap S \in \operatorname{Syl}_{p}(S)$, and since $S / Z$ is good for the prime $p$, there exists a correspondence subgroup $T=\mathbf{T}(S, Q \cap S, \nu)$, and an associated character correspondence ( )* from $\operatorname{Irr}_{p^{\prime}}(S \mid \nu)$ to $\operatorname{Irr}_{p^{\prime}}(T \mid \nu)$. Since we are holding $Q$ and $\nu$ fixed throughout this discussion, we can remove some clutter from our notation and simply write $T=\mathbf{T}(S)$. (As usual, we call all of our character correspondences ( )*.)

We can now define the subgroup $H$ of $K$ appearing in Theorem 13.1 by setting $H=$ $\prod_{S \in \mathcal{S}} \mathbf{T}(S)$. Note that $H$ is a central product of the subgroups $\mathbf{T}(S)$, and it is easy to see that $\mathbf{T}(S)=H \cap S$ for each member $S \in \mathcal{S}$. The following lemma establishes the purely group-theoretic parts of Theorem 13.1 for this subgroup $H$.

(13.2) LEMMA. Let $K, Q$ and $H$ be as above. Then

(a) $\mathbf{N}_{K}(Q) \subseteq H<K$.

Now suppose that $K$ is normal in some group $G$ with $Z \subseteq \mathbf{Z}(G)$, and write $N=\mathbf{N}_{G}(H)$. The following then hold.

(b) $K N=G$ and $K \cap N=H$.

(c) $\mathbf{N}_{G}(Q) \subseteq N<G$.

Proof. Since $S \cap H=\mathbf{T}(S)<S$, we have $H<K$, as required. To complete the proof of (a), let $k \in \mathbf{N}_{K}(Q)$ and observe that $k$ normalizes each of the subgroups $Q \cap S$ for $S \in \mathcal{S}$. Now $k$ is a product of elements of the various members $S$ of $\mathcal{S}$, and each component of $k$, except possibly the $S$-component, centralizes $S$ and thus normalizes $Q \cap S$. It follows that the $S$-component of $k$ must also normalize $Q \cap S$. We know, however, that $\mathbf{N}_{S}(Q \cap S) \subseteq \mathbf{T}(S, Q \cap S, \nu)=\mathbf{T}(S)$, and it follows that $k \in \prod_{S \in \mathcal{S}} \mathbf{T}(S)=H$. Thus $\mathbf{N}_{K}(Q) \subseteq H$, proving (a).

Now suppose $K \triangleleft G$ with $Z \subseteq \mathbf{Z}(G)$, and let $M=\mathbf{N}_{G}(Q)$. If $m \in M$ and $S \in \mathcal{S}$, then $S^{m} \in \mathcal{S}$ and conjugation by $m$ defines an isomorphism from $S$ to $S^{m}$ that is trivial on $Z$ and carries $Q \cap S$ to $Q \cap S^{m}$. It follows that conjugation by $m$ maps $\mathbf{T}(S)$ to $\mathbf{T}\left(S^{m}\right)$, and thus $M$ permutes the subgroups $\mathbf{T}(S)$ for $S \in \mathcal{S}$. We conclude that $M$ normalizes $H$, proving part of (c).

By the Frattini argument, $G=M K \subseteq N K$, and so $G=N K$, as wanted. Also $N \cap K=\mathbf{N}_{K}(H)=H$, where the second equality holds because $H$ contains a Sylow normalizer in $K$. This proves (b). Also, since $N \cap K=H<K$, we have $N<G$, and this completes the proof of (c). 
We need a map $\theta \mapsto \theta^{*}$ from $\operatorname{Irr}_{p^{\prime}}(K \mid \nu)$ to $\operatorname{Irr}_{p^{\prime}}(H \mid \nu)$. To construct it, it is convenient to enumerate the members of $\mathcal{S}$, and so we write $\mathcal{S}=\left\{S_{1}, S_{2}, \ldots, S_{e}\right\}$, and we set $T_{i}=\mathbf{T}\left(S_{i}\right)$. Since $K$ is the central product of the $S_{i}$, it follows by Lemma 5.1 that every character $\theta \in \operatorname{Irr}(K \mid \nu)$ is uniquely of the form $\theta=\theta_{1} \bullet \theta_{2} \cdots \cdot \theta_{e}$, with $\theta_{i} \in \operatorname{Irr}\left(S_{i} \mid \nu\right)$. Similarly, $H$ is the central product of the $T_{i}$, and all members of $\operatorname{Irr}(H \mid \nu)$ can be obtained by taking dot products of characters in $\operatorname{Irr}\left(T_{i} \mid \nu\right)$. Given $\theta \in \operatorname{Irr}_{p^{\prime}}(K \mid \nu)$, the factors $\theta_{i}$ lie in $\operatorname{Irr}_{p^{\prime}}\left(S_{i} \mid \nu\right)$, and so the characters $\theta_{i}^{*} \in \operatorname{Irr}_{p^{\prime}}\left(T_{i} \mid \nu\right)$ are defined. We now set $\theta^{*}=\theta_{1}^{*} \bullet \theta_{2}^{*} \cdots \cdot \theta_{e}^{*}$, and it should be clear that this defines a bijection from $\operatorname{Irr}_{p^{\prime}}(K \mid \nu)$ to $\operatorname{Irr}_{p^{\prime}}(H \mid \nu)$.

Now let $A$ be the group of automorphisms of $K$ that stabilize $Q$ and centralize $Z$.

(13.3) LEMMA. The map $\theta \mapsto \theta^{*}$ defined above is A-equivariant. Also, Part (d) of Theorem 13.1 holds for this map.

Proof. Let $a \in A$ and observe that $a$ permutes the subgroups $S \in \mathcal{S}$. If $a$ carries $S_{i}$ to $S_{j}$, then it carries $T_{i}$ to $T_{j}$ and also, if $\theta_{i} \in \operatorname{Irr}_{p^{\prime}}\left(S_{i} \mid \nu\right)$, then $\left(\theta_{i}\right)^{a} \in \operatorname{Irr}_{p^{\prime}}\left(S_{j} \mid \nu\right)$. Since $a$ fixes $\nu$ and carries $Q \cap S_{i}$ to $Q \cap S_{j}$, we know that $\left(\left(\theta_{i}\right)^{*}\right)^{a}=\left(\left(\theta_{i}\right)^{a}\right)^{*}$, and it follows easily that ( $)^{*}$ is $A$-equivariant, as desired.

Now assume the situation of Theorem 13.1 and write $N=\mathbf{N}_{G}(H)$ and $M=\mathbf{N}_{G}(Q)$. By Lemma 13.2(c), we have $M \subseteq N$, and by the Frattini argument, $N=M H$. To prove that the map ( $)^{*}$ is $N$-equivariant, therefore, it suffices to prove that it is $M$-equivariant. But $M$ induces automorphisms in $A$, and so this is clear.

The following is the analog of Theorems 11.1 and 12.1 in this situation.

(13.4) THEOREM. Let $K, H, Q$ and $\nu$ be as above. Let $\theta \in \operatorname{Irr}_{p^{\prime}}(K \mid \nu)$ and suppose that $(G, N, C)$ is a relatedness candidate for $\left(\theta, \theta^{*}\right)$. Then $\theta$ and $\theta^{*}$ are related in $(G, N, C)$.

Proof. We work by induction on the number $e=|\mathcal{S}|$. The set $\mathcal{S}$ is permuted by $G$, and we assume first that this action is not transitive. We can then write $K$ as a central product, $K=K_{1} K_{2}$, where $K_{i} \triangleleft G$ and each of $K_{1}$ and $K_{2}$ is the product of fewer than $e$ members of the set $\mathcal{S}$. In particular, we have $Z=\mathbf{Z}\left(K_{i}\right)$ and $K_{1} \cap K_{2}=Z$, and thus we can write $\theta=\theta_{1} \bullet \theta_{2}$, where $\theta_{i} \in \operatorname{Irr}_{p^{\prime}}\left(K_{i} \mid \nu\right)$.

Each of the groups $K_{i}$ satisfies the original hypotheses on $K$ (with a smaller value of $e$ ), and so there exist subgroups $H_{i} \subseteq K_{i}$ defined analogously to the construction of $H$ in $K$. Also, we have associated bijections ( $)^{*}$ from $\operatorname{Irr}_{p^{\prime}}\left(K_{i} \mid \nu\right)$ to $\operatorname{Irr}_{p^{\prime}}\left(H_{i} \mid \nu\right)$. We see that $H=H_{1} H_{2}$ is a central product with $H_{1} \cap H_{2}=Z$, and we have $H_{i}=H \cap K_{i}$. Furthermore, it follows from the construction of the various ( ) maps that $\theta^{*}=\theta_{1}^{*} \bullet \theta_{2}^{*}$.

Since $(G, N, C)$ is a relatedness candidate for $\left(\theta, \theta^{*}\right)$, we know that $\theta$ and $\theta^{*}$ are $N$-invariant, and it is immediate that each of $\theta_{1}, \theta_{2}, \theta_{1}^{*}$ and $\theta_{2}^{*}$ is also $N$-invariant. Also, there exists a $G$-invariant character $\gamma \in \operatorname{Irr}(C \mid \nu)$, and our goal is to show that $[\theta \cdot \gamma]_{G / K C}=$ $\left[\theta^{*} \cdot \gamma\right]_{N / H C}$. Observe that $K C=K_{1} K_{2} C$ is the central product of the three subgroups $K_{1}$, $K_{2}$ and $C$, and thus we can write $\theta \cdot \gamma=\left(\theta_{1} \bullet \theta_{2}\right) \cdot \gamma=\theta_{1} \bullet \theta_{2} \bullet \gamma$, and there is no ambiguity caused by dropping the parentheses. Similarly, we can write $\theta^{*} \bullet \gamma=\theta_{1}^{*} \bullet \theta_{2}^{*} \bullet \gamma$

The subgroup $H_{i}$ is self-normalizing in $K_{i}$, and so if we write $N_{i}=\mathbf{N}_{G}\left(H_{i}\right)$, we see that $N_{1} \cap K=H_{1} K_{2}$ and $N_{2} \cap K=K_{1} H_{2}$. Also $N_{1} \cap N_{2}=\mathbf{N}_{G}(H)=N$. Furthermore, since $G=N K$ and $N \subseteq N_{1}$, we have $N_{1}=N\left(N_{1} \cap K\right)=N H_{1} K_{2}=N K_{2}$, and similarly, 
$N_{2}=N K_{1}$. It follows that $N_{1} K_{1}=N K_{1} K_{2}=N K=G$, and similarly, $N_{2} K_{2}=G$. The groups $G / K, N_{1} / H_{1} K_{2}, N_{2} / K_{1} H_{2}$ and $N / H$ are naturally isomorphic, and it is easy to see that four groups $G / C K, N_{1} / C H_{1} K_{2}, N_{2} / C K_{1} H_{2}$ and $N / C H$ are also naturally isomorphic, and so we can identify them.

Now $\left(K_{1}, H_{1}, \nu\right)$ is a strong triple, and we observe that $\left(G, N_{1}, C K_{2}\right)$ lies over it. To see this, we must check that $N_{1} K_{1}=G$, that $N_{1} \cap K_{1}=H_{1}$, that $C K_{2}$ centralizes $K_{1}$ and that $K_{1} \cap C K_{2}=Z$. All of this is clear.

We argue that $\left(G, N_{1}, C K_{2}\right)$ is a relatedness candidate for $\left(\theta_{1}, \theta_{1}^{*}\right)$. To see this, observe that since $\theta_{1}$ is $G$-invariant, it is certainly $N_{1}$-invariant, and thus $\theta_{1}^{*}$ is also $N_{1}$-invariant, as required. Furthermore, we know that $\gamma \in \operatorname{Irr}(C \mid \nu)$ is $G$-invariant. Since $C K_{2}$ is a central product with $C \cap K_{2}=Z$, the character $\gamma \bullet \theta_{2} \in \operatorname{Irr}\left(C K_{2} \mid \nu\right)$ is defined and $G$-invariant, and this shows that $\left(G, N_{1}, C K_{2}\right)$ is a relatedness candidate for $\left(\theta_{1}, \theta_{1}^{*}\right)$, as asserted.

By the inductive hypothesis applied with $K_{1}$ in place of $K$, it follows that $\theta_{1}$ and $\theta_{1}^{*}$ are related in $\left(G, N_{1}, C K_{2}\right)$, and thus

$$
\left[\theta_{1} \bullet\left(\gamma \cdot \theta_{2}\right)\right]_{G / C K}=\left[\theta_{1}^{*} \bullet\left(\gamma \cdot \theta_{2}\right)\right]_{N_{1} / C H_{1} K_{2}} \cdot
$$

Since $C K=C K_{1} K_{2}$ and $C H_{1} K_{2}$ are triple central products, we can suppress the parentheses and rearrange the factors in the above character dot products. We thus have

$$
\left[\theta_{1} \cdot \theta_{2} \bullet \gamma\right]_{G / C K}=\left[\theta_{1}^{*} \bullet \theta_{2} \cdot \gamma\right]_{N_{1} / C H_{1} K_{2}}
$$

Next, we observe that $\left(N_{1}, N, C H_{1}\right)$ lies over the strong triple $\left(K_{2}, H_{2}, \nu\right)$. For this we must check that $N K_{2}=N_{1}$, that $N \cap K_{2}=H_{2}$, that $C H_{1}$ centralizes $K_{2}$, and that $C H_{1} \cap K_{2}=Z$. Here too, all of this is clear.

In fact, $\left(N_{1}, N, C H_{1}\right)$ is a relatedness candidate for $\left(\theta_{2}, \theta_{2}^{*}\right)$. That $\theta_{2}$ and $\theta_{2}^{*}$ are $N$ invariant is clear, and we have the $N_{1}$-invariant character $\gamma \bullet \theta_{1}^{*} \in \operatorname{Irr}\left(C H_{1} \mid \nu\right)$, as needed.

Now we apply the inductive hypothesis in the group $N_{1}$, with $K_{2}$ in place of $K$, and we deduce that $\theta_{2}$ and $\theta_{2}^{*}$ are related in $\left(N_{1}, N, C H_{1}\right)$. We thus have

$$
\left[\theta_{2} \bullet\left(\gamma \cdot \theta_{1}^{*}\right)\right]_{N_{1} / C H_{1} K_{2}}=\left[\theta_{2}^{*} \cdot\left(\gamma \cdot \theta_{1}^{*}\right)\right]_{N / C H},
$$

and again, we can suppress parentheses and rearrange factors. We obtain

$$
\left[\theta_{1}^{*} \cdot \theta_{2} \bullet \gamma\right]_{N_{1} / C H_{1} K_{2}}=\left[\theta_{1}^{*} \cdot \theta_{2}^{*} \cdot \gamma\right]_{N / C H}
$$

Combining this with our previous equality of cohomology elements, we get

$$
[\theta \cdot \gamma]_{G / C K}=\left[\theta_{1} \bullet \theta_{2} \bullet \gamma\right]_{G / C K}=\left[\theta_{1}^{*} \bullet \theta_{2} \bullet \gamma\right]_{N_{1} / C H_{1} K_{2}}=\left[\theta_{1}^{*} \bullet \theta_{2}^{*} \cdot \gamma\right]_{N / C H}=\left[\theta^{*} \cdot \gamma\right]_{N / C H}
$$

and thus $\theta$ and $\theta^{*}$ are related in $(G, N, C)$, as required.

We can now assume that $G$ acts transitively on the set $\mathcal{S}$. (In particular, the base case of our induction, where $e=1$, is included here.) Again, it is convenient to enumerate the set $\mathcal{S}$, and we write $\mathcal{S}=\left\{S_{1}, \ldots, S_{e}\right\}$. As before, we write $\theta=\theta_{1} \cdot \cdots \cdot \theta_{e}$ with $\theta_{i} \in \operatorname{Irr}_{p^{\prime}}\left(S_{i} \mid \nu\right)$, and we observe that the character $\theta_{i}$ can be identified as the unique 
irreducible constituent of $\theta_{S_{i}}$. Also, by definition, $\theta^{*}=\theta_{1}^{*} \cdot \cdots \cdot \theta_{e}^{*}$, and so $\theta_{i}^{*}$ is the unique irreducible constituent of $\left(\theta^{*}\right)_{T_{i}}$, where we have written $T_{i}=\mathbf{T}\left(S_{i}\right)$.

By Corollary 8.3, we know that to prove that $\theta$ and $\theta^{*}$ are related in $(G, N, C)$, it is no loss to replace $(G, N, C)$ with a relatedness candidate equivalent to it. By Lemma 9.4(c) and (d), therefore, we can can assume that $G$ splits over $K$. In fact, we can assume that there is a complement $R$ containing a normal subgroup that acts (by conjugation) on $K$ like the group of inner automorphisms of $K$. (Note that this change of our group $G$ does not affect the set of automorphisms of $K$ induced by $G$, and thus $G$ is still transitive on S.)

Next, as we did in Section 5, we build the group $K^{*}$ as the (external) direct product of the groups $S_{i}$. Viewing each factor as a subgroup of $K^{*}$, the centers $Z_{i}$ of the subgroups $S_{i}$ are distinct subgroups of $K^{*}$, each of them isomorphic to $Z$. Define subgroups $Z^{*}$ and $H^{*}$ of $K^{*}$ by setting $Z^{*}=\prod Z_{i}$ and $H^{*}=\prod T_{i}$. Note that $Z^{*}=\mathbf{Z}\left(K^{*}\right)$ and $Z^{*} \subseteq H^{*}$. Also, since $T_{i}$ is self-normalizing in $S_{i}$, we see that $H^{*}$ is self-normalizing in $K^{*}$.

The natural homomorphism from $K^{*}$ onto $K$, mapping an $e$-tuple in $K^{*}$ to the product of its components in $K$, carries $H^{*}$ to $H$ and $Z^{*}$ to $Z$. We can thus view the characters $\theta \in \operatorname{Irr}(K), \theta^{*} \in \operatorname{Irr}(H)$ and $\nu \in \operatorname{Irr}(Z)$ as characters of $K^{*}, H^{*}$ and $Z^{*}$, respectively. Note that $\left(K^{*}, H^{*}, \nu\right)$ is a strong triple, but $\nu$ is no longer faithful.

The conjugation action of the complement $R$ on $K$ in $G$ induces a natural action of $R$ on the direct product $K^{*}$, and we let $G^{*}=K^{*} R$ be the semidirect product. It is easy to check that there is a unique surjection from $G^{*}$ to $G$ that extends both the natural surjection from $K^{*}$ to $K$ and the identity map on $R$. If we write $N^{*}=\mathbf{N}_{G^{*}}\left(H^{*}\right)$, we see that $N^{*}$ is the full preimage in $G^{*}$ of $N=\mathbf{N}_{G}(H)$. (This is because the kernel of our homomorphism from $G^{*}$ to $G$ is contained in $Z^{*} \subseteq H^{*}$.) Also, since $G=K N$, it follows that $G^{*}=K^{*} N^{*}$, and we have $K^{*} \cap N^{*}=\mathbf{N}_{K_{*}}\left(H^{*}\right)=H^{*}$. Furthermore, since $\theta$ and $\theta^{*}$ are invariant under the action of $N$, we see that $\theta$ and $\theta^{*}$ are also invariant under $N^{*}$.

We argue next that the full preimage $C^{*}$ of $C$ in $G^{*}$ centralizes $K^{*}$. To see this, note that each element of $G^{*}$ has the form $x r$, with $x \in K^{*}$ and $r \in R$. If $x r$ lies in $C^{*}$, then the image of $x r$ in $G$ lies in $C$, and it has the form $y r$, where $y$ is the image of $x$ in $K$. Now $y r$ centralizes $K$, and so $r^{-1}$ induces on $K$ the inner automorphism induced by $y$. In particular, $r^{-1}$ normalizes each member $S \in \mathcal{S}$. Also, $r^{-1}$ induces on $S$ the automorphism induced by $y$, which is the same as the automorphism induced by $x$. It follows that the element $x r$ of $C^{*}$ centralizes each factor $S$ of $K^{*}$, and thus $C^{*}$ centralizes $K^{*}$, as claimed. Thus $C^{*}=\mathbf{C}_{G^{*}}\left(K^{*}\right)$ and $C^{*} \cap K^{*}=\mathbf{Z}\left(K^{*}\right)=Z^{*}$. We see, therefore, that $\left(G^{*}, N^{*}, C^{*}\right)$ lies over the strong triple $\left(K^{*}, H^{*}, \nu\right)$. We know that $\left(\theta, \theta^{*}\right)$ is invariant in $\left(G^{*}, N^{*}, C^{*}\right)$, and in fact, we claim that $\left(G^{*}, N^{*}, C^{*}\right)$ is a relatedness candidate for $\left(\theta, \theta^{*}\right)$. This is because the given $G$-invariant character $\gamma$ of $C$ can be viewed as a $G^{*}$-invariant character of $C^{*}$ lying over $\nu$.

We work now to show that the characters $\theta$ and $\theta^{*}$ are related in $\left(G^{*}, N^{*}, C^{*}\right)$. This will complete the proof because in general, if $M \triangleleft G$ and $\psi \in \operatorname{Irr}(M)$ is $G$-invariant, then the cohomology element $[\psi]_{G / M}$ is the same as the cohomology element that would be obtained if we first factored out by a normal subgroup of $G$ contained in $\operatorname{ker}(\psi)$.

Let $F=\mathbf{C}_{G^{*}}\left(K^{*}\right)$ and observe that $F \subseteq \mathbf{N}_{G^{*}}\left(H^{*}\right)=N^{*}$. Corollary 9.2 applies in this situation, and since $\operatorname{Irr}\left(K^{*} \mid \nu\right)$ contains the $R$-invariant character $\theta$, it follows that 
$\operatorname{Irr}(F \mid \nu)$ also contains an $R$-invariant character. But $G^{*}=K^{*} R$ and $K^{*}$ centralizes $F$, and hence every $R$-invariant character of $F$ is also $G^{*}$-invariant. It follows that $\left(G^{*}, N^{*}, F\right)$ is a relatedness candidate for $\left(\theta, \theta^{*}\right)$, and so by Theorem 7.1 (Going Down), it suffices to show that $\theta$ and $\theta^{*}$ are related in $\left(G^{*}, N^{*}, F\right)$. (We will explain later why we have to abandon $C^{*}$ and work with $F$ instead.)

Since $K^{*}$ is the direct product of its subgroups $S_{i}$, we can (uniquely) factor $\theta=\prod \xi_{i}$, where $\xi_{i} \in \operatorname{Irr}\left(K^{*}\right)$ and $S_{j} \subseteq \operatorname{ker}\left(\xi_{i}\right)$ for $j \neq i$. Also $\xi_{i}$ restricts irreducibly to $S_{i}$ and we can identify $\left(\xi_{i}\right)_{S_{i}}$ as the unique irreducible constituent of $\theta_{S_{i}}$. In particular, we have $\left(\xi_{i}\right)_{S_{i}}=\theta_{i}$. Also, since $H^{*}$ is the direct product of its subgroups $T_{i}$, we can apply similar reasoning, and we write $\theta^{*}=\prod \eta_{i}$, where $\eta_{i} \in \operatorname{Irr}\left(H^{*}\right)$ and $T_{j} \subseteq \operatorname{ker}\left(\eta_{i}\right)$ for $j \neq i$. We have $\left(\eta_{i}\right)_{T_{i}}=\theta_{i}^{*}$.

Factor $\nu=\prod \lambda_{i}$, where $\lambda_{i} \in \operatorname{Irr}\left(Z^{*}\right)$ and $Z_{j} \subseteq \operatorname{ker}\left(\lambda_{i}\right)$ for $j \neq i$. Let $\mu_{i}=\left(\lambda_{i}\right)_{Z_{i}}$. (Recall that each of the groups $Z_{i}$ is "really" our original group $Z$. From this point of view, the character $\mu_{i}$ is just our original character $\nu$, but since we have changed the definition of $\nu$, which we now view as a character of $Z^{*}$, we need to distinguish $\mu_{i}$ from $\nu$.) Observe that $\xi_{i} \in \operatorname{Irr}\left(K^{*} \mid \lambda_{i}\right)$ and that $\eta_{i} \in \operatorname{Irr}\left(H^{*} \mid \lambda_{i}\right)$.

Now $R$ transitively permutes the set $\mathcal{S}$, and we write $R_{1}$ to denote the stabilizer of $S_{1}$ in this action. Working in $G^{*}$, we set $G_{1}=K^{*} R_{1}$ and $N_{1}=N^{*} \cap G_{1}$, and we observe that $G_{1}=K^{*} N_{1}$ and $H^{*}=K^{*} \cap N_{1}$. Also, $G_{1}=\mathbf{N}_{G^{*}}\left(S_{1}\right)$, and hence $F=\mathbf{C}_{N^{*}}\left(K^{*}\right) \subseteq N_{1}$. Furthermore, we see that $G_{1}$ is the stabilizer of $\xi_{1}$ in $G^{*}$ and $N_{1}$ is the stabilizer of both $\xi_{1}$ and $\eta_{1}$ in $N^{*}$. Thus $\left(G_{1}, N_{1}, F\right)$ lies over the strong triple $\left(K^{*}, H^{*}, \lambda_{1}\right)$ and $\left(\xi_{1}, \eta_{1}\right)$ belongs to this triple and is invariant in $\left(G_{1}, N_{1}, F\right)$.

We claim that $\left(G_{1}, N_{1}, F\right)$ is a relatedness candidate for $\left(\xi_{1}, \eta_{1}\right)$. To see this, we must show that there is a $G_{1}$-invariant character in $\tau \in \operatorname{Irr}\left(F \mid \lambda_{1}\right)$. But $G_{1}=K^{*} R_{1}$ and $K^{*}$ centralizes $F$, and so it suffices to find an $R_{1}$-invariant character $\operatorname{in} \operatorname{Irr}\left(F \mid \lambda_{1}\right)$. The existence of such a character is guaranteed by Corollary 9.2 , however, since $\operatorname{Irr}\left(K^{*} \mid \lambda_{1}\right)$ contains the $R_{1}$-invariant character $\xi_{1}$. (Note that we could not apply 9.2 if we were working with $C^{*}$ instead of $F$, and so it is not clear that $\left(G_{1}, N_{1}, C\right)$ is a relatedness candidate for $\left(\xi_{1}, \eta_{1}\right)$.)

Recall that $N_{1}$ is the stabilizer of both $\xi_{1}$ and $\eta_{1}$ in $N^{*}$. Also, we know that $\theta$ is the product of the $e=\left|N^{*}: N_{1}\right|$ distinct $N^{*}$-conjugates of $\xi_{1}$ and $\theta^{*}$ is the product of the $e$ distinct $N^{*}$-conjugates of $\eta_{1}$. It follows by Theorem 7.2 that to prove that $\theta$ and $\theta^{*}$ are related in $\left(G^{*}, N^{*}, F\right)$, it suffices to show that $\xi_{1}$ and $\eta_{1}$ are related in $\left(G_{1}, N_{1}, F\right)$. To complete the proof, therefore, it suffices to show that the cohomology elements $\left[\xi_{1} \bullet \tau\right]_{G_{1} / K^{*} F}$ and $\left[\eta_{1} \bullet \tau\right]_{N_{1} / H^{*} F}$ are equal. For notational convenience now, we write $\xi=\xi_{1}$ and $\eta=\eta_{1}$.

Observe that $\left(K^{*} F\right) N_{1}=G_{1}$ and $\left(K^{*} F\right) \cap N_{1}=H^{*} F$. Let $N_{1} \subseteq M \subseteq G_{1}$ and set $D=\left(K^{*} F\right) \cap M$, so that $M / D$ is naturally isomorphic to both $G_{1} / K^{*} F$ and $N_{1} / H^{*} F$. We will choose an appropriate subgroup $M$ and an appropriate $M$-invariant character $\delta \in \operatorname{Irr}(D)$ such that

$$
[\xi \cdot \tau]_{G_{1} / K^{*} F}=[\delta]_{M / D}=[\eta \cdot \tau]_{N_{1} / H^{*} F},
$$

and this will establish the desired equality of cohomology elements.

Let $M=\mathbf{N}_{G_{1}}\left(T_{1}\right)$. Now $T_{1}=H^{*} \cap S_{1}$, and since $N_{1}$ normalizes both $H^{*}$ and $S_{1}$, we have $N_{1} \subseteq M \subseteq G_{1}$, as wanted. To compute $D=K^{*} F \cap M$ conveniently, we define $L=\prod_{j>1} S_{j}$. Then $K^{*}=S_{1} \times L$ and since $T_{1}$ is self-normalizing in $S_{1}$, we see that 
$\mathbf{N}_{K^{*}}\left(T_{1}\right)=T_{1} L$. This yields

$$
D=K^{*} F \cap M=\left(K^{*} \cap M\right) F=\mathbf{N}_{K^{*}}\left(T_{1}\right) F=T_{1} L F .
$$

Next, observe that $L \cap F=\prod_{j>1} Z_{j}$. Then $L \cap F \subseteq \operatorname{ker}\left(\lambda_{1}\right)$, and hence $L \cap F \subseteq$ $\operatorname{ker}(\tau)$. It follows that $\tau$ has a unique extension $\sigma \in \operatorname{Irr}(L F)$ such that $L \subseteq \operatorname{ker}(\sigma)$. Now $D=T_{1}(L F)$ is a central product of $T_{1}$ and $L F$, and we see that $T_{1} \cap L F=Z_{1}$. Also, both $\theta_{1}^{*}$ and $\sigma$ lie over $\mu_{1} \in \operatorname{Irr}\left(Z_{1}\right)$, and so $\theta_{1}^{*} \cdot \sigma \in \operatorname{Irr}(D)$ is defined, and we take $\delta=\theta_{1}^{*} \cdot \sigma$. Note that $\delta$ is $M$-invariant because it is $N_{1}$-invariant.

Now $K^{*}=S_{1} L$, and so $K^{*} F=S_{1}(L F)$, and this is a central product of $S_{1}$ and $L F$. Also, $S_{1} \cap L F=Z_{1}$ and both $\theta_{1} \in \operatorname{Irr}\left(S_{1}\right)$ and $\sigma \in \operatorname{Irr}(L F)$ lie over $\mu_{1} \in \operatorname{Irr}\left(Z_{1}\right)$. Thus $\theta_{1} \bullet \sigma$ is a well defined character of $K^{*} F$, and we argue that $\theta_{1} \bullet \sigma=\xi \bullet \tau$. To establish this, let $x \in K^{*} F$ and write $x=s l f$, where $s \in S_{1}, l \in L$ and $f \in F$. Then

$$
\left(\theta_{1} \bullet \sigma\right)(x)=\theta_{1}(s) \sigma(l f)=\xi(s l) \tau(f)=(\xi \bullet \tau)(x),
$$

as wanted. (The second equality holds because $L \subseteq \operatorname{ker}(\xi)$ and $\xi_{S_{1}}=\theta_{1}$.)

Next, we observe that $\left(G_{1}, M, L F\right)$ lies over the strong triple $\left(S_{1}, T_{1}, \mu_{1}\right)$. Also, $\sigma \in$ $\operatorname{Irr}\left(L F \mid \mu_{1}\right)$ is uniquely determined by $\tau$, and so it is $G_{1}$-invariant, and hence $\left(G_{1}, M, L F\right)$ is a relatedness candidate for $\left(\theta_{1}, \theta_{1}^{*}\right)$. But $T_{1}=\mathbf{T}\left(S_{1}\right)$, and so by Theorem 12.1, we see that $\theta_{1}$ and $\theta_{1}^{*}$ are related in $\left(G_{1}, M, \mu_{1}\right)$. Recalling that $K^{*} F=S_{1}(L F)$, and that $\xi \bullet \tau=\theta_{1} \bullet \sigma$, we see that

$$
[\xi \cdot \tau]_{G_{1} / K^{*} F}=\left[\theta_{1} \bullet \sigma\right]_{G_{1} / S_{1}(L F)}=\left[\theta_{1}^{*} \cdot \sigma\right]_{M / T_{1}(L F)}=[\delta]_{M / D},
$$

as wanted.

Finally, to show that $[\delta]_{M / D}=[\eta \cdot \tau]_{N_{1} / H^{*} F}$, it suffices by Lemma 3.6 to show that $\delta$ restricts irreducibly to $H^{*} F$ and that the restriction is equal to $\eta \bullet \tau$. Let $x \in H^{*} F$ and note that $H^{*}=T_{1}\left(H^{*} \cap L\right)$. Write $x=t l f$, where $t \in T_{1}, l \in H^{*} \cap L$ and $f \in F$. Then

$$
\delta(x)=\left(\theta_{1}^{*} \cdot \sigma\right)(t l f)=\theta_{1}^{*}(t) \sigma(l f)=\eta(t l) \tau(f)=(\eta \bullet \tau)(x),
$$

as wanted. (Here, the third equality holds because $H^{*} \cap L \subseteq \operatorname{ker}(\eta), \eta_{T_{1}}=\theta_{1}^{*}$ and $\sigma(l f)=\tau(f)$ by the definition of $\sigma$.) This completes the proof.

Proof of Theorem 13.1 Assume the notation of Theorem 13.1, let $H$ be as before, and let $\theta \in \operatorname{Irr}_{p^{\prime}}(K \mid \nu)$. All that remains to be shown is that $\left|\operatorname{Irr}_{p^{\prime}}(G \mid \theta)\right|=\left|\operatorname{Irr}_{p^{\prime}}\left(N \mid \theta^{*}\right)\right|$.

Let $G_{0}$ be the stabilizer of $\theta$ in $G$ and write $N_{0}=G_{0} \cap N$. By Conclusion (d) of the theorem, which has already been established, we know that $N_{0}$ is the stabilizer in $N$ of $\theta^{*}$. Also, character induction defines bijections from $\operatorname{Irr}\left(G_{0} \mid \theta\right)$ to $\operatorname{Irr}(G \mid \theta)$ and from $\operatorname{Irr}\left(N_{0} \mid \theta^{*}\right)$ to $\operatorname{Irr}\left(N \mid \theta^{*}\right)$. Now $\left|G: G_{0}\right|=\left|N: N_{0}\right|$ by Conclusion (b), and if this number is divisible by $p$, then both $\operatorname{Irr}_{p^{\prime}}(G \mid \theta)$ and $\operatorname{Irr}_{p^{\prime}}\left(N \mid \theta^{*}\right)$ are empty, and there is nothing further to prove. We may suppose, therefore, that the stabilizer indices are not divisible by $p$, and hence induction defines bijections from $\operatorname{Irr}_{p^{\prime}}\left(G_{0} \mid \theta\right)$ to $\operatorname{Irr}_{p^{\prime}}(G \mid \theta)$ and from $\operatorname{Irr}_{p^{\prime}}\left(N_{0} \mid \theta^{*}\right)$ to $\operatorname{Irr}_{p^{\prime}}\left(N \mid \theta^{*}\right)$. It suffices, therefore, to show that $\left|\operatorname{Irr}_{p^{\prime}}\left(G_{0} \mid \theta\right)\right|=\left|\operatorname{Irr}_{p^{\prime}}\left(N_{0} \mid \theta^{*}\right)\right|$. 
Now $\left(G_{0}, N_{0}, Z\right)$ lies over $(K, H, \nu)$ and is clearly a relatedness candidate for $\left(\theta, \theta^{*}\right)$, where the $G_{0}$-invariant character in $\operatorname{Irr}(Z \mid \nu)$ is, of course, $\nu$ itself. We know by Theorem 13.4 that $\theta$ and $\theta^{*}$ are related in $\left(G_{0}, N_{0}, Z\right)$, and thus $[\theta]_{G_{0} / K}=\left[\theta^{*}\right]_{N_{0} / H}$. (We are using the fact that $\theta \cdot \nu=\theta$ and similarly for $\theta^{*}$.) It follows from the theory of projective representations that there is a bijection from $\operatorname{Irr}\left(G_{0} \mid \theta\right)$ to $\operatorname{Irr}\left(N_{0} \mid \theta^{*}\right)$ such that if $\chi$ corresponds to $\chi^{*}$, then $\chi(1) / \theta(1)=\chi^{*}(1) / \theta^{*}(1)$. In particular, since $\theta(1)$ and $\theta^{*}(1)$ are not divisible by $p$, this bijection carries $\operatorname{Irr}_{p^{\prime}}\left(G_{0} \mid \theta\right)$ to $\operatorname{Irr}_{p^{\prime}}\left(N_{0} \mid \theta^{*}\right)$. This completes the proof of the theorem.

\section{Fields and automorphisms.}

In this short section, we prove the following result, which we shall need when we show that the simple groups $\mathrm{PSL}_{2}(\mathrm{q})$ are good for all primes that divide their orders.

(14.1) THEOREM. Let $E$ be a finite field and write $E^{\times}$to denote its multiplicative group. If $S$ is a group of automorphisms of $E$, then the Schur multiplier of the semidirect product $\left(E^{\times}\right) \rtimes S$ is trivial.

We begin by recalling a general and standard lemma.

(14.2) LEMMA. Assume that $A \triangleleft G$, where $A$ is abelian and $G / A$ is cyclic. Then $\left|G^{\prime}\right|=|A: A \cap \mathbf{Z}(G)|$.

Our theorem will follow from the following general result, which can be used to show that for certain metacyclic groups $G$, the Schur multipliers $M(G)$ are trivial. In particular, we mention that this lemma shows that the multipliers of semidihedral and generalized quaternion groups are trivial.

(14.3) LEMMA. Let $G=C B$, where $B$ and $C$ are cyclic and $C \triangleleft G$. If $B$ contains $C \cap \mathbf{Z}(G)$, then $M(G)=1$.

Proof. Clearly, $C \cap B \subseteq C \cap \mathbf{Z}(G)$, and so by hypothesis, we have equality here. By Lemma 14.2, therefore, $\left|G^{\prime}\right|=|C: C \cap B|$.

Our goal is to show that if $G=H / Z$, where $Z \subseteq H^{\prime} \cap \mathbf{Z}(H)$, then $Z=1$. In this situation, let $V$ and $U$, respectively, be the preimages in $H$ of $C$ and $B$. Then $U$ and $V$ are abelian and $U V=H$, and thus $V \cap U \subseteq \mathbf{Z}(H)$. Also, $V \triangleleft H$ and $H / V$ is cyclic. Since $H^{\prime} / Z=G^{\prime}$, Lemma 14.2 yields

$$
|Z|\left|G^{\prime}\right|=\left|H^{\prime}\right|=|V: V \cap \mathbf{Z}(H)| \leq|V: V \cap U|=|C: C \cap B|=\left|G^{\prime}\right|
$$

and the result follows.

We also need the following standard result from field theory, which can be proved using Hilbert's Theorem 90. (See the note following Problem 23.8 of [10].)

(14.4) LEMMA. Let $F \subseteq E$ be finite fields. Then the norm map $E^{\times} \rightarrow F^{\times}$is surjective. 
Proof of Theorem 14.1. Since $E^{\times}$and $S$ are cyclic, we can choose generators and write $E^{\times}=\langle e\rangle$ and $S=\langle s\rangle$. Let $F$ be the fixed field of $S$ in $E$ and write $m=|S|$. Now let $Y=\langle s e\rangle$ and note that $Y$ is cyclic and $G=E^{\times} Y$. By Lemma 14.3, therefore, it suffices to show that $Y$ contains $E^{\times} \cap \mathbf{Z}(G)=\mathbf{C}_{E^{\times}}(S)=F^{\times}$.

Now $(s e)^{m}=s^{m} e^{s^{m-1}} e^{s^{m-2}} \cdots e^{s} e$, and since $s^{m}=1$, we see that $(s e)^{m}$ is the norm of $e$ in $F^{\times}$. But $e$ generates $E^{\times}$, and since the image of the norm map is $F^{\times}$, it follows that $(s e)^{m}$ generates $F^{\times}$. Thus $F^{\times} \subseteq Y$, as wanted.

\section{The groups $\operatorname{PSL}_{2}(q)$.}

We are now ready to begin studying specific simple groups, working toward a proof of Theorem A. We show first that simple groups of the form $\operatorname{PSL}_{2}(q)$ are "good" in the sense of Section 10 (for all primes dividing their order). Since every simple group involved in a group of this type is again of this type, it will follow, as we explained in the introduction, that the McKay conjecture holds for all primes for groups in which all nonabelian composition factors are of the form $\mathrm{PSL}_{2}(q)$. In the following two sections, we will handle the Suzuki groups $\mathrm{Sz}\left(2^{e}\right)$ and the Ree groups $\mathrm{R}\left(3^{e}\right)$, thereby completing the proof of Theorem A.

Let $q=r^{f}$, where $r$ is prime, and let $X=\operatorname{PSL}_{2}(q)$ with $q \geq 5$. (Recall that the groups $\mathrm{PSL}_{2}(q)$ are solvable for $q \leq 3$ and $\mathrm{PSL}_{2}(4) \cong \mathrm{PSL}_{2}(5)$.) In order to prove that $X$ is good for all prime divisors $p$ of its order, we need to verify certain properties of the irreducible characters of the various covering groups of $X$, as in Section 10 . Note that apart from the case $q=9$, when $X$ is isomorphic to the alternating group $A_{6}$, the group $\mathrm{SL}_{2}(q)$ (which we denote $S$ ) is the universal covering group of $X$, and thus it suffices to consider the irreducible characters of $S$. The exceptional cases of faithful characters of $3 \cdot A_{6}$ and $6 \cdot A_{6}$ are dealt with later.

(15A) The irreducible characters of $\mathrm{GL}_{2}(q)$ and $\mathrm{SL}_{2}(q)$.

We start by recalling the parametrization of irreducible characters of $\mathrm{GL}_{2}(q)$ and $\mathrm{SL}_{2}(q)$. The group $\widetilde{G}=\mathrm{GL}_{2}(q)$ has two conjugacy classes of maximal tori. A representative $\widetilde{H}_{1}$ of the first class consists of the diagonal matrices in $\widetilde{G}$, while a representative $\widetilde{H}_{2}$ of the second class can be obtained by embedding $\mathbb{F}_{q^{2}}^{\times}$via its linear action (by multiplication) on the $\mathbb{F}_{q^{-}}$-vector space $\mathbb{F}_{q^{2}}$ of dimension 2. In particular, $\left|\widetilde{H}_{1}\right|=(q-1)^{2}$ and $\left|\widetilde{H}_{2}\right|=q^{2}-1$. The intersection $\widetilde{H}_{1} \cap \widetilde{H}_{2}$ is independent of the particular conjugates chosen, and coincides with the center $\mathbf{Z}(\widetilde{G})$, consisting of all scalar matrices.

We now choose a group $\widetilde{G}^{*}$ in duality with $\widetilde{G}$, and in $\widetilde{G}^{*}$, we choose maximal tori $\widetilde{H}_{i}^{*}$ in duality with $\widetilde{H}_{i}$ for $i=1,2$. It is well known that in our situation, $\widetilde{G}^{*} \cong \widetilde{G}=\mathrm{GL}_{2}(q)$. (See for example Section 4.4 of [2].) Also, we have induced isomorphisms $\delta_{i}: \operatorname{Irr}\left(\widetilde{H}_{i}\right) \rightarrow \widetilde{H}_{i}^{*}$. (See Proposition 4.4.1 of [2].) We may (and will) use these isomorphisms to identify $\widetilde{H}_{i}^{*}=\operatorname{Irr}\left(\widetilde{H}_{i}\right)$, and we think of elements $s \in \widetilde{H}_{i}^{*}$ as irreducible characters of $\widetilde{H}_{i}$.

Note that each semisimple element of $\widetilde{G}^{*}$ is conjugate to an element of $\widetilde{H}_{1}^{*} \cup \widetilde{H}_{2}^{*}$. Let $s \in \widetilde{H}_{i}^{*}$. According to the fundamental construction of Deligne and Lusztig, there is an associated generalized character $\widetilde{R}_{i}(s)$ of $\widetilde{G}$, defined by $\widetilde{R}_{i}(s)=R_{\widetilde{H}_{i}}^{\widetilde{G}}(s)$, and this depends only on the $\widetilde{G}^{*}$-class of $s$ and the choice of $i$. (See Section 7 of [2].) 
Furthermore, $\pm \widetilde{R}_{i}(s)$ is irreducible if and only if the centralizer of $s$ in $\widetilde{G}^{*}$ is exactly the maximal torus $\widetilde{H}_{i}^{*}$, or equivalently, if $s \notin \mathbf{Z}\left(\widetilde{G}^{*}\right)$. (See Corollary 7.3.5 of [2].) If $s \in \mathbf{Z}\left(\widetilde{G}^{*}\right)$ then $\widetilde{R}_{i}(s)$ has two irreducible constituents: an extension of the principal character of $\mathrm{SL}_{2}(q)$ to $\widetilde{G}$, and an extension of the Steinberg character of $\mathrm{SL}_{2}(q)$ to $\widetilde{G}$, the later having degree $q$. Thus in particular,

$$
\pm \widetilde{R}_{i}:\left(\widetilde{H}_{i}^{*} \backslash \mathbf{Z}\left(\widetilde{G}^{*}\right)\right) \rightarrow \operatorname{Irr}(\widetilde{G}), \quad i=1,2,
$$

are 2-to-1 maps, with two elements having the same image if and only if they are conjugate under the normalizer $\mathbf{N}_{\widetilde{G}^{*}}\left(\widetilde{H}_{i}^{*}\right)$.

Now let $S^{*}=\operatorname{PGL}_{2}(q)$ and let $\pi: \widetilde{G}^{*} \rightarrow S^{*}$ denote the natural epimorphism. Then $S^{*}$ is in duality with the subgroup $S=\mathrm{SL}_{2}(q)$ of $\mathrm{GL}_{2}(q)$, and $H_{i}^{*}=\pi\left(\widetilde{H}_{i}^{*}\right)$ is in duality with $H_{i}=\widetilde{H}_{i} \cap \mathrm{SL}_{2}(q)$ for $i=1,2$. In particular $\pi$ induces identifications $\operatorname{Irr}\left(H_{i}\right)=H_{i}^{*}$.

Again, each semisimple element of $S^{*}$ is conjugate to an element of $H_{1}^{*} \cup H_{2}^{*}$. To $s \in H_{i}^{*}$, there is associated a Deligne-Lusztig generalized character $R_{i}(s)=R_{H_{i}}^{S}(s)$, and we have the following compatibility with respect to restriction:

$$
\widetilde{R}_{i}(s)_{\mathrm{SL}_{2}(q)}=R_{i}(\pi(s)) \quad \text { for all } s \in \widetilde{H}_{i}^{*}, i=1,2 .
$$

This restriction $R_{i}(\pi(s))$ is (up to sign) an irreducible character of $S$ if and only if $\widetilde{R}_{i}(s)$ itself is irreducible and in addition, the preimages under $\pi$ of $\pi(s)$ lie in $q-1$ different conjugacy classes. (See [12].) Note that $\widetilde{R}_{i}(s)$ is irreducible precisely when $s \notin \mathbf{Z}\left(\widetilde{G}^{*}\right)$. Also, an easy calculation shows that the preimages of $\pi(s)$ lie in distinct classes if and only if the order of $\pi(s)$ is larger than 2 . In this case, the characters $\widetilde{R}_{i}(t)$ with $\pi(t)=\pi(s)$ are the different extensions of $R_{i}(\pi(s))$ to $\widetilde{G}$. We then write $\chi_{\pi(s)}$ for the irreducible character $\pm R_{i}(\pi(s))$. Thus in particular

$$
\pm R_{i}:\left(H_{i}^{*} \backslash\left\{s \mid s^{2}=1\right\}\right) \rightarrow \operatorname{Irr}(S), \quad i=1,2,
$$

are 2-to-1 maps, where two elements have the same image if and only if they are conjugate under the normalizer $\mathbf{N}_{S^{*}}\left(H_{i}^{*}\right)$. The generalized characters $R_{i}(1)$ have two irreducible constituents: the principal character $1_{S}$ and the Steinberg character St of degree $q$. These are the so-called unipotent characters of $S$. If $q$ is odd, $S^{*}$ contains two classes of involutions, one with representative $a \in H_{1}^{*}$, the other with representative $b \in H_{2}^{*}$. Then $R_{1}(a)=\chi_{a}^{+}+\chi_{a}^{-}$has two irreducible constituents of degree $(q+1) / 2$ each, and similarly $-R_{2}(b)=\chi_{b}^{+}+\chi_{b}^{-}$has two irreducible constituents of degree $(q-1) / 2$ each. In this way we have obtained a parametrization of $\operatorname{Irr}(S)$ via a map from $\operatorname{Irr}(S)$ to the set of conjugacy classes in $S^{*}$. The fibers of this map have size at most two, and all but at most three of the fibers have size one.

The data on $\operatorname{Irr}(S)$ are collected in Table 1, below. Note that the semisimple elements of order dividing $q \pm 1$ are conjugate to their inverses in $S^{*}$; this explains the numbers of characters given in the last two columns.

TABLE 1. Irreducible characters of $\mathrm{SL}_{2}(q)$. 


\begin{tabular}{|lc|c|c|c|}
\hline \multicolumn{1}{|c|}{$\theta$} & $\theta(1)$ & number $(q$ odd $)$ & number $(q$ even $)$ \\
\hline \hline $1_{G}$ & 1 & 1 & 1 \\
\hline St & $q$ & 1 & 1 \\
\hline$\chi_{s}$ & $s^{q-1}=1, s^{2} \neq 1$ & $q+1$ & $\frac{1}{2}(q-3)$ & $\frac{1}{2}(q-2)$ \\
\hline$\chi_{t}$ & $t^{q+1}=1, t^{2} \neq 1$ & $q-1$ & $\frac{1}{2}(q-1)$ & $\frac{1}{2} q$ \\
\hline$\chi_{a}^{ \pm}$ & $\frac{1}{2}(q+1)$ & 2 & 0 \\
\hline$\chi_{b}^{ \pm}$ & $\frac{1}{2}(q-1)$ & 2 & 0 \\
\hline
\end{tabular}

Next, for odd $q$, we describe the restrictions of irreducible characters of $\widetilde{G}$ and $S$ to the respective centers. By Section 8 of $[\mathbf{1 2}]$, there is a natural isomorphism $\widetilde{G}^{*} /\left[\widetilde{G}^{*}, \widetilde{G}^{*}\right] \cong$ $\operatorname{Irr}(\mathbf{Z}(\widetilde{G}))$, and the restriction to $\mathbf{Z}(\widetilde{G})$ is then the composite with the natural map

$$
\operatorname{Irr}\left(\widetilde{H}_{i}\right) \cong \widetilde{H}_{i}^{*} \rightarrow \widetilde{H}_{i}^{*} /\left(\widetilde{H}_{i}^{*} \cap\left[\widetilde{G}^{*}, \widetilde{G}^{*}\right]\right) .
$$

Thus the restriction map

$$
\widetilde{z}: \widetilde{H}_{1}^{*} \cup \widetilde{H}_{2}^{*} \rightarrow \operatorname{Irr}(\mathbf{Z}(\widetilde{G}))
$$

to $\mathbf{Z}(\widetilde{G})$ factors through $\widetilde{G}^{*} /\left[\widetilde{G}^{*}, \widetilde{G}^{*}\right]$, so that the restriction of $s$ only depends on the determinant, that is, the constant coefficient of the characteristic polynomial of $s$.

Since Deligne-Lusztig induction preserves central characters, we have that $\widetilde{R}_{i}(s)$ also lies over $\widetilde{z}(s)$, see [12]. Clearly $\pi(s)$ lies over the restriction $\widetilde{z}(s)_{\mathbf{Z}(S)}$, so the map giving central characters for $S$ is just the determinant modulo squares. Since we saw that $R_{i}(\pi(s))=\widetilde{R}_{i}(s)_{S}$, it follows that $R_{i}(\pi(s))$ lies over $\widetilde{z}(s)_{\mathbf{Z}(S)}$. In particular, $\chi_{s}$ is faithful on $\mathbf{Z}(S)$ if and only if $\operatorname{det}\left(\pi^{-1}(s)\right)$ is not a square, that is, if $s$ lies outside of the subgroup $\mathrm{PSL}_{2}(q)$ of $\mathrm{PGL}_{2}(q)$, or equivalently, when $s$ is not a square in $\mathrm{PGL}_{2}(q)$. For example, $\chi_{a}^{ \pm}$ is faithful precisely when $q \equiv 3(\bmod 4)$, and $\chi_{b}^{ \pm}$is faithful precisely when $q \equiv 1(\bmod 4)$.

(15B) Automorphisms and extendibility.

Next, we describe the action of outer automorphisms on the irreducible characters of $X$ and $S$. We write $\widehat{G}=\Gamma L_{2}(q)$ for the extension of $\mathrm{GL}_{2}(q)$ by the group of field automorphisms Aut $\left(\mathbb{F}_{q}\right)$ acting on the entries of matrices in $\mathrm{GL}_{2}(q)$. Then $S$ is normal in $\widehat{G}$, and $\widehat{G}$ induces the full group of automorphisms on both $S$ and $X=S / \mathbf{Z}(S)$. Indeed, for $q$ even, $X=S$ has cyclic outer automorphism group consisting of the field automorphisms induced by $\operatorname{Aut}\left(\mathbb{F}_{q}\right)$, while for odd $q$, the outer automorphism group of $X$ is generated by the field automorphisms together with the diagonal automorphism induced by the embedding of $X=\mathrm{PSL}_{2}(q)$ into $\mathrm{PGL}_{2}(q)$. In any case, $\operatorname{Aut}(X) \cong \mathrm{P}_{2}(q)=\widehat{G} / \mathbf{Z}\left(\mathrm{GL}_{2}(q)\right)$.

Recall that $\Sigma \mathrm{L}_{2}(q)$ is the subgroup of $\Gamma \mathrm{L}_{2}(q)$ generated by $\mathrm{SL}_{2}(q)$ and the group of field automorphisms $\operatorname{Aut}\left(\mathbb{F}_{q}\right)$. Note also that $\widehat{G} / S \cong \mathbb{F}_{q}^{\times} \cdot \operatorname{Aut}\left(\mathbb{F}_{q}\right)$ is metacyclic. (In fact, it is cyclic if $q=r$ ).

(15.1) LEMMA. Let $\theta \in \operatorname{Irr}(S)$ and denote by $\widehat{G}_{\theta}$ the inertia group of $\theta$ in $\widehat{G}$. If $\theta \in\left\{\chi_{a}^{ \pm}, \chi_{b}^{ \pm}\right\}$then $\widehat{G}_{\theta}=\Sigma \mathrm{L}_{2}(q) \mathbf{Z}\left(\mathrm{GL}_{2}(q)\right)$, while otherwise $\mathrm{GL}_{2}(q) \subseteq \widehat{G}_{\theta}$. 
Proof. By Lusztig's construction of the irreducible characters as constituents of suitable Deligne-Lusztig-characters, the cyclic group $\operatorname{Aut}\left(\mathbb{F}_{q}\right) \cong \Gamma L_{2}(q) / \mathrm{GL}_{2}(q)$ of field automorphisms acts on characters $\chi_{s}^{( \pm)}$of $S$ via their labels, that is, on the coefficients of their characteristic polynomials. (See [12].) Thus, in particular, it fixes the unipotent characters 1 and St of $S$, as well as the characters $\chi_{a}^{ \pm}$and $\chi_{b}^{ \pm}$.

The diagonal automorphisms are induced by the embedding of $S$ into $\mathrm{GL}_{2}(q)$. By what we said in Section $(15 \mathrm{~A}) \chi_{s}^{( \pm)}$extends to $\mathrm{GL}_{2}(q)$ if and only if the class of $s$ in $\mathrm{PGL}_{2}(q)$ has precisely $q-1$ preimages under the natural map $\pi: \mathrm{GL}_{2}(q) \rightarrow \mathrm{PGL}_{2}(q)$. Thus by our above parametrization, the outer diagonal automorphism of order 2 just interchanges $\chi_{a}^{+}$with $\chi_{a}^{-}$and $\chi_{b}^{+}$with $\chi_{b}^{-}$. It fixes all other irreducible characters of $S$. The claim follows.

We now study the extendibility of a character $\theta \in \operatorname{Irr}(S)$ to its inertia group in $\widehat{G}=\Gamma L_{2}(q)$.

(15.2) LEMMA. Let $\theta \in \operatorname{Irr}(S)$. Then the inertia factor group of $\theta$ in $\widehat{G}$ has trivial Schur multiplier, and in particular, $\theta$ extends to its inertia group $\widehat{G}_{\theta}$ in $\widehat{G}$.

Proof. By Theorem 11.7 of [9], we are done if we can show that the inertia factor group of $\theta$ has trivial Schur multiplier. For $\theta \in\left\{\chi_{a}^{ \pm}, \chi_{b}^{ \pm}\right\}$the inertia factor group $\widehat{G}_{\theta} / S$ is cyclic, and the claim follows from Corollary 11.21 of [9].

For the remaining characters $\theta$, we saw above that the inertia group is an extension of $\mathrm{GL}_{2}(q)$ with a group of field automorphisms, and hence the inertia factor group is an extension of $\widehat{S} / S \cong \mathbb{F}_{q}^{\times}$with a group of field automorphisms. By Theorem 14.1, every such group has trivial Schur multiplier

The case $q=9$ illustrates an interesting point. Here, $S=\mathrm{SL}_{2}(9) \cong 2 \cdot A_{6}$, the 2 -fold cover of the alternating group $A_{6}$. Now $A_{6}$ has a unique irreducible character $\theta$ of degree 10 , which we view as a (nonfaithful) character of $S$. The uniqueness of $\theta$ guarantees that it is invariant in $\Gamma L_{2}(9)$, and in particular, by Lemma 15.1 (or by the data in Table 1) we see that $\theta$ is not one of the characters $\chi_{a}^{ \pm}$or $\chi_{b}^{ \pm}$. (In fact, $\theta=\chi_{s}$, where $s \in \mathrm{PSL}_{2}(9)$ has order 4.) Thus $\widehat{G}_{\theta}=\Gamma L_{2}(9)$. In this case, the inertia factor group is semidihedral of order 16 and $\theta$ extends to $\widehat{G}_{\theta}$ by Lemma 15.2 .

On the other hand, if we view $\theta$ as a character of $A_{6}$ instead of $2 \cdot A_{6}$, it is well known that $\theta$ does not extend to $\operatorname{Aut}\left(A_{6}\right)$. (Here the inertia factor group is the Klein 4-group, with nontrivial Schur multiplier.) This example shows that the passage to the larger group $\Gamma L_{2}(9)$ can simplify questions of extendibility.

We can now begin the proof of the main result of this section.

(15.3) THEOREM. Let $X=\operatorname{PSL}_{2}(q)$, where $q=r^{f} \geq 4$. Then the simple group $X$ is good for all primes $p$ that divide its order.

Proof. According to the definitions in Section 10, for each prime $p$ dividing $|X|$ and each irreducible character $\theta$ of a covering group of $X$, we have to produce a group $G=G(\theta)$ satisfying certain conditions. As we remarked at the beginning of this section, there is only one non-trivial covering group of $X$, namely $S=\mathrm{SL}_{2}(q)$ for $q$ odd. (The exceptional 
covering groups in the case $q=9$ will be treated separately at the end of the proof.) Instead of treating the faithful characters of $S$ and the characters of $X$ in two separate arguments, we consider the latter characters as (non-faithful) characters of $S$, and we check both cases at once. Thus, our notation here will be such that for any fixed character $\theta \in \operatorname{Irr}(S)$ the groups $S, G, T, N$ and $C$ occurring in Section 10 are the quotients by the kernel of $\theta$ of the groups with the same name in this proof. Also, we will denote the character bijections with $\iota$ since the notation ()$^{*}$ is being used to denote dual groups. We hope that this slight deviation from the notation in Section 10 will not cause confusion.

Given $\theta \in \operatorname{Irr}(S)$, we let $G(\theta)=\widehat{G}_{\theta}$. Thus, in the notation of Section 10, $G=$ $G(\theta) / \operatorname{ker}(\theta)$. Let $C=\mathbf{C}_{G(\theta)}(S)$, and note that $C$ is a subgroup of $\mathbf{Z}\left(\mathrm{GL}_{2}(q)\right)$, and hence is cyclic. This establishes condition (6) of Section 10. By Lemma 15.1 above, $G(\theta)$ is the full inertia group of $\theta$ in $\widehat{G}$, which in turn induces the full automorphism group of $S$, proving condition (5).

Now $\theta$ extends to $G(\theta)$ by Lemma 15.2 , and we let $\widehat{\theta}$ denote such an extension. Let $\nu \in \operatorname{Irr}(\mathbf{Z}(S))$ lie under $\theta$, and let $\mu \in \operatorname{Irr}(C)$ lie under $\widehat{\theta}$. Then clearly $\mu$ is a $G(\theta)$-invariant extension of $\nu$. Furthermore, $\widehat{\theta}$ is an extension of $\theta \cdot \mu$ to $G(\theta)$, as required in $(7)$.

Now for all prime divisor $p$ of $|S|$ we are left with two tasks. First, we must construct a subgroup $T$ of $S$ containing the normalizer $\mathbf{N}_{S}(Q)$ of a Sylow $p$-subgroup $Q$ of $S$ satisfying conditions (1) and (2). Note that

$$
\mathbf{N}_{\widehat{G} / \mathbf{Z}(S)}(Q \mathbf{Z}(S) / \mathbf{Z}(S))=\mathbf{N}_{\widehat{G}}(Q) / \mathbf{Z}(S)
$$

for all primes $p$, so that conditions (1) and (2) are satisfied for $T$ in $S$ if and only if they are satisfied for $T / \mathbf{Z}(S)$ in $X$. Secondly, we need to establish an $A$-equivariant bijection

$$
\iota_{p}: \operatorname{Irr}_{p^{\prime}}(S) \rightarrow \operatorname{Irr}_{p^{\prime}}(T)
$$

between the characters of $S$ with $p^{\prime}$-degree and those of $T$ with $p^{\prime}$-degree, where $A=$ $\mathbf{N}_{\widehat{G}}(Q)$, and we must verify condition (8). In order to establish (8) we will show that all characters in $\operatorname{Irr}_{p^{\prime}}(T)$ extend to their inertia groups in $\mathbf{N}_{\widehat{G}}(T)$. (This is only an issue when $q$ is odd, since otherwise, $\operatorname{Out}(X)$ is cyclic.)

We have $|S|=q\left(q^{2}-1\right)$, and we will subdivide into cases according to whether $p$ divides $q-1, q+1$ or $q$, and we will treat the case $p=2$ separately.

(15C) Odd primes dividing $q-1$.

Let $p$ be an odd prime divisor of $q-1$. Then the Sylow $p$-subgroup $Q$ of the maximal torus $H_{1}$ of order $q-1$ consisting of the diagonal matrices in $S$ is also a Sylow $p$-subgroup of $S$. Its normalizer $T=\mathbf{N}_{S}(Q)$ is an extension of $H_{1}$ by the cyclic group of order 2 , acting by inversion. By construction, $T$ satisfies conditions (1) and (2). Note that all irreducible characters of $T$ have degree 1 or 2 , and so they have $p^{\prime}$-degree.

Recall our identification of the irreducible characters of $H_{1}$ with the dual torus $H_{1}^{*} \cong$ $\mathbb{F}_{q}^{\times}$of $S^{*}=\mathrm{PGL}_{2}(q)$. The characters of order at most 2 in $H_{1}^{*}$ are precisely those that extend to $T$, while all others induce to characters of degree 2 . Thus, for $q$ odd we have four linear characters $1^{+}, 1^{-}, \psi_{a}^{+}$, and $\psi_{a}^{-}$in $\operatorname{Irr}(T)$ and also $(q-3) / 2$ induced characters $\psi_{s}=(s)^{T} \in \operatorname{Irr}(T)$ of degree 2 , indexed by $s \in H_{1}^{*}$ (modulo $T$-conjugation), with $s^{2} \neq 1$. 
For even $q$, we have two linear characters $1^{+}$and $1^{-}$and $(q-2) / 2$ induced characters $\psi_{s}=(s)^{T}$ of degree 2 in $\operatorname{Irr}(T)$. Hence induction from $H_{1}$ to $T$ defines a 2-to-1 map

$$
\left(H_{1}^{*} \backslash\left\{s \mid s^{2}=1\right\}\right) \rightarrow \operatorname{Irr}(T) .
$$

The restriction of $\psi_{s}$ to $\mathbf{Z}(S)$ is a multiple of the restriction of $s$ to $\mathbf{Z}(S)$, and thus $\psi_{s}^{( \pm)}$ has $\mathbf{Z}(S)$ in its kernel if and only if $s$ is a square.

On the other hand, by Table 1 , the characters of $S$ of $p^{\prime}$-degree are the unipotent characters and also the characters $\chi_{s}^{( \pm)}$, with $s \in H_{1}^{*}$ (modulo inversion). We obtain an obvious bijection

$$
\iota_{p}: \operatorname{Irr}_{p^{\prime}}(S) \rightarrow \operatorname{Irr}(T)
$$

by sending

$$
\chi_{s} \mapsto \psi_{s}, \quad \chi_{a}^{ \pm} \mapsto \psi_{a}^{ \pm}, \quad 1 \mapsto 1^{+}, \quad \mathrm{St} \mapsto 1^{-},
$$

and this respects central characters, as required by condition (3). Moreover $\iota_{p}$ satisfies congruences modulo $q-1$ (hence modulo $p$ ) for the degrees.

Note that by the construction of the characters of degree $q+1$, the map $\iota_{p}$ is related to Deligne-Lusztig induction in the following way

$$
\iota_{p}\left(R_{H_{1}}^{S}(s)\right)=(s)^{T} \quad\left(s \in H_{1}^{*}, s^{2} \neq 1\right)
$$

under our identification of $H_{1}^{*}$ with $\operatorname{Irr}\left(H_{1}\right)$.

What remains is to verify conditions (4) and (8) for the bijection $\iota_{p}$. We first prove equivariance with respect to $A=\mathbf{N}_{\widehat{G}}(Q)$. It is clear by our description of $\operatorname{Irr}(T)$ that the field automorphisms act on $\operatorname{Irr}(T)$ via the labels, and thus by the corresponding description for $\operatorname{Irr}(S)$, the bijection $\iota_{p}$ is equivariant with respect to field automorphisms.

We now turn to diagonal automorphisms (which exist only if $q$ is odd). These are induced by the embedding of $S$ into $\mathrm{GL}_{2}(q)$. Here, the normalizer $\widetilde{T}=\mathbf{N}_{\mathrm{GL}_{2}(q)}(Q)$ of $Q$ is the normalizer of the maximal torus $\widetilde{H}_{1}$, which has order $(q-1)^{2}$ and consists of all diagonal matrices in $\mathrm{GL}_{2}(q)$, and so $\widetilde{T}$ is a wreath product $C_{q-1} / C_{2}$. All degree 2 characters of $T$ extend to $\widetilde{T}$, while the two linear characters $\psi_{a}^{ \pm}$fuse. The action of the diagonal automorphisms is thus compatible with $\iota_{p}$. Thus $\iota_{p}$ preserves inertia subgroups, and this proves (4).

Now fix a character $\theta=R_{H_{1}}^{S}(s) \in \operatorname{Irr}(S)$ of degree $q+1$. Then $\theta$ extends to a character $\widehat{\theta}$ of $\widehat{G}_{\theta}$. Let $\widetilde{\theta}$ denote the restriction of $\widehat{\theta}$ to $\mathrm{GL}_{2}(q) \subseteq \widehat{G}_{\theta}$, so $\widetilde{\theta}$ is an extension of $\theta$ to $\mathrm{GL}_{2}(q)$. Write $C=\mathbf{Z}\left(\mathrm{GL}_{2}(q)\right)=\mathbf{C}_{\widehat{G}_{\theta}}(S)$ and note that condition $(6)$ is satisfied since $C$ is abelian. Now $\widetilde{\theta}$ is an irreducible Deligne-Lusztig character of $\mathrm{GL}_{2}(q)$, and hence there exists an irreducible character $\widetilde{s} \in \widetilde{H}_{1}^{*}=\operatorname{Irr}\left(\widetilde{H}_{1}\right)$ with

$$
\widetilde{\theta}=R_{\widetilde{H}_{1}}^{\mathrm{GL}_{2}(q)}(\widetilde{s}) .
$$

Since $\widetilde{\theta}$ extends $\theta=R_{H_{1}}^{S}(s)$, it follows that $\widetilde{s}$ maps to $s$ under the canonical surjection $\mathrm{GL}_{2}(q) \rightarrow \mathrm{PGL}_{2}(q)$, i.e., $\widetilde{s} \in \widetilde{H}_{1}^{*}=\operatorname{Irr}\left(\widetilde{H}_{1}\right)$ extends $s \in H_{1}^{*}=\operatorname{Irr}\left(H_{1}\right)$. By $(15 \mathrm{~A}), \widetilde{\theta}$ 
lies over the central character $\gamma=\widetilde{z}(\widetilde{s})$, which then is invariant in $\widehat{G}_{\theta}$, as required by condition (7). Moreover, $\widehat{\theta}$ is an extension of $\theta \cdot \gamma$ to $\widehat{G}_{\theta}$. Thus $\psi_{\widetilde{s}}=(\widetilde{s})^{\widetilde{T}}$ is an extension of $(s)^{T}=\iota_{p}(\theta)$ to $\widetilde{T}$ lying over $\gamma$. To verify condition (8), therefore, it suffices to show that $\iota_{p}(\theta) \cdot \gamma$ extends to its stabilizer $A_{\theta}$ in $A$.

Since $\psi_{\widetilde{s}}=(\widetilde{s})^{\widetilde{T}}$ is invariant in $A_{\theta}$ and $A_{\theta} / \widetilde{T}$ is cyclic, it follows that $\psi_{\widetilde{s}}$ extends to $A_{\theta}$, and thus $\iota_{p}(\theta) \cdot \gamma$ also extends to $A_{\theta}$. This establishes (8) and completes the proof for odd primes $p$ dividing $q-1$.

(15D) Odd primes dividing $q+1$.

The case of odd primes $p$ dividing $q+1$ is very similar to the previous one. We replace the maximal torus $H_{1}$ by a maximal torus $H_{2}$ of $S$ of order $q+1$, isomorphic to the subgroup of norm 1 elements in $\mathbb{F}_{q^{2}}^{\times}$. We omit the details.

(15E) The prime $p=2$.

Now let $p=2 \neq r$, so that the Sylow 2-subgroup $Q$ of $S$ is a quaternion group. First assume $q \equiv 1(\bmod 8)$. Then $Q$ is self-normalizing in $S$ (and $Q / \mathbf{Z}(S)$ is self-normalizing in $X)$. Instead of considering this group, we take $T$ to be the normalizer $T=\mathbf{N}_{S}\left(H_{1}\right)$ of a maximal torus containing $Q$. It is easy to verify that the bijection established above for odd prime divisors $p$ of $(q-1)$ preserves characters of $2^{\prime}$-degree, and thus we are done by the previous considerations. (Note that this makes sense even if $q-1$ is a power of 2 , so that in fact there does not exist an odd prime $p$ dividing $q-1$.)

If $q \equiv 7(\bmod 8)$, the argument is rather similar: we take $T=\mathbf{N}_{S}\left(H_{2}\right)$, where $H_{2}$ is the other maximal torus, and we proceed as before.

If $q \equiv 5(\bmod 8)$, then $Q$ is quaternion of order 8 , with normalizer $T=\mathbf{N}_{S}(Q)$ isomorphic to $\mathrm{SL}_{2}(3)$, and so $T$ has four irreducible characters of $2^{\prime}$-degree. On the other hand the characters of $S$ of odd degree are 1 , St and $\chi_{a}^{ \pm}$. The diagonal automorphisms in $A$ extend $T$ to $\mathrm{GL}_{2}(3)$, and thus only two of its four characters of $2^{\prime}$-degree are invariant under diagonal automorphisms. On the other hand, $T$ is centralized by all field automorphisms. Thus we obtain an obvious $A$-equivariant bijection from $\operatorname{Irr}_{p^{\prime}}(S)$ to $\operatorname{Irr}_{p^{\prime}}(T)$ in this case.

Finally, if $q \equiv 3(\bmod 8)$, we again take $T=\mathbf{N}_{S}(Q)$, and again $T$ is isomorphic to $\mathrm{SL}_{2}(3)$. In this case, the four irreducible characters of $2^{\prime}$-degree of $S$ are 1 , St, and $\chi_{b}^{ \pm}$.

(15F) The prime $p=r$. Now let $p=r$, the defining characteristic. (For groups with connected center like $\mathrm{GL}_{2}(q)$, this case was first looked at by Green, Lehrer and Lusztig in [7].) In all previous cases, there was a natural candidate for the required bijection, coming from the fact that Lusztig's parametrization of characters by semisimple classes in the dual group works both for $S$ as well as for the centralizer of a Sylow subgroup. In defining characteristic, there does not seem to be such a natural map, and things become more complicated.

The subgroup $U$ of $S$ consisting of the upper unitriangular matrices is a Sylow $r$ subgroup of $S$, with normalizer the Borel subgroup $B$ of invertible upper triangular matrices. Here $U \cong \mathbb{F}_{q}^{+}$is elementary abelian of order $q=r^{f}$, and $B / U \cong \mathbb{F}_{q}^{\times}$acts by multiplication with squares:

$$
\mathbb{F}_{q}^{+} \times \mathbb{F}_{q}^{\times} \rightarrow \mathbb{F}_{q}^{+}, \quad(u, t) \mapsto t^{2} u
$$


In any case, $B / U \mathbf{Z}(S)$ acts semi-regularly on the non-trivial characters of $U$. Thus $B$ has $q-1$ linear characters, trivial on $U$, and either one character $\lambda_{0}$ of degree $q-1$ when $q$ is even, or four characters $\lambda_{0}^{1,2,3,4}$ of degree $(q-1) / 2$ when $q$ is odd.

Note that $B / U$ is isomorphic to the maximal torus $H_{1}$ of $S$, so the linear characters of $B$ may be regarded as characters of $H_{1}$, hence as elements of $H_{1}^{*}$. We also write $\lambda_{s}$ for the irreducible character of $B$ which equals $s \in H_{1}^{*}$ on $H_{1}$. Thus $\lambda_{s}$ is trivial on the center $\mathbf{Z}(S) \subseteq B$ if and only if $s$ is a square in $H_{1}^{*}$, and precisely two of the four characters $\lambda_{0}^{1,2,3,4}$ are trivial on $\mathbf{Z}(S)$. We summarize the description of $\operatorname{Irr}(B)$ in Table 2. Here, "faithful" means "faithful on $\mathbf{Z}(S)$ ". The last three columns show the number of characters in each case.

TABLE 2. Irreducible characters of $B$.

\begin{tabular}{|l|c|c|c|c|}
\hline \multicolumn{1}{|c|}{$\theta$} & $\theta(1)$ & nonfaithful $(q$ odd $)$ & faithful ( $q$ odd) & $(q$ even $)$ \\
\hline \hline$\lambda_{s} \quad s \in \mathbb{F}_{q}^{\times}$ & 1 & $\frac{1}{2}(q-1)$ & $\frac{1}{2}(q-1)$ & $q-1$ \\
\hline$\lambda_{0}$ & $q-1$ & 0 & 0 & 1 \\
\hline$\lambda_{0}^{1,2,3,4}$ & $\frac{1}{2}(q-1)$ & 2 & 2 & 0 \\
\hline
\end{tabular}

We proceed to check the conditions in Section 10. Let $T=B=\mathbf{N}_{S}(U)$, the Borel subgroup. Then (1) and (2) are satisfied. Comparison with the situation in $S$, where $\operatorname{Irr}_{p^{\prime}}(S)=\operatorname{Irr}(S)-\{\mathrm{St}\}$, shows that the numbers of irreducible $p^{\prime}$-degree characters of $T$ and $S$ are equal.

First assume that $q$ is odd. We construct a $\mathbf{N}_{\widehat{G}}(T)$-equivariant bijection

$$
\iota: \operatorname{Irr}_{p^{\prime}}(T) \rightarrow \operatorname{Irr}_{p^{\prime}}(S)
$$

as follows. The four induced characters $\lambda_{0}^{1,2,3,4}$ are mapped to $\chi_{a}^{ \pm}$and $\chi_{b}^{ \pm}$. Since exactly two of $\lambda_{0}^{1,2,3,4}$ and of $\chi_{a}^{ \pm}$and $\chi_{b}^{ \pm}$are faithful on $\mathbf{Z}(S)$, this is possible in a way such that central characters are respected. To define $\iota\left(\lambda_{s}\right)$, note that the polynomials

$$
\left\{x^{2}+2 x+s \mid s \in \mathbb{F}_{q}^{\times}\right\}
$$

constitute a set of representatives (modulo rescaling) of the degree 2 polynomials

$$
x^{2}+\mu_{1} x+\mu_{2} \in \mathbb{F}_{q}[x],
$$

where $\mu_{1}$ and $\mu_{2}$ are nonzero. We then let $\iota\left(\lambda_{s}\right)=\chi_{t}$ where $t \in H_{1}^{*} \cup H_{2}^{*}$ has characteristic polynomial $g_{s}=x^{2}+2 x+s$. By our previous observation, this establishes a bijection from the linear characters of $B$ to the semisimple characters of $S$.

We now compare the corresponding central characters. As remarked above, $\lambda_{s}$ is trivial on $\mathbf{Z}(S)$ if and only if $s$ is a square. We thus need to see that $g_{s}$ labels a class consisting of squares if and only if $s$ itself is a square. If $g_{s}$ splits over $\mathbb{F}_{q}$, then $g_{s}$ labels 
a class of squares if and only if the ratio of its roots is a square in $\mathbb{F}_{q}^{\times}$. If $g_{s}$ is irreducible over $\mathbb{F}_{q}$, it labels a class of squares if and only if the ratio of its roots in $\mathbb{F}_{q^{2}}$ is a square in the group of norm 1 units of this field.

In the case where $g_{s}$ splits, its roots $\alpha$ and $\beta$ lie in $\mathbb{F}_{q}$, and we see that $s=\alpha \beta=$ $\beta^{2}(\alpha / \beta)$, is a square if and only if $\alpha / \beta$ is a square, as required. Assume now that $g_{s}$ is irreducible and let $\beta$ be one of its roots in $\mathbb{F}_{q^{2}}$. Then $\beta^{q}$ is the other root, and their ratio is $\beta^{q-1}$. It suffices to show, therefore, that $s$ is a square in $\mathbb{F}_{q}$ if and only if $\beta^{(q-1) / 2}$ has norm 1 , or equivalently, that $\beta^{(q-1)(q+1) / 2}=1$. But $s=\beta^{q+1}$, and so $\beta^{(q-1)(q+1) / 2}=$ $s^{(q-1) / 2}$, and this is equal to 1 if and only if $s$ is a square, as wanted.

Next we consider the action of field automorphisms. On the linear characters, which are just the characters of $B / U \cong \mathbb{F}_{q}^{\times}$, the action is via the label $s$, and this is the same action as on the associated polynomial $g_{s}$. On the other hand, the induced characters are clearly invariant, as are $\chi_{a}^{ \pm}, \chi_{b}^{ \pm}$.

For odd $q$ the diagonal automorphisms of $B$ are induced by the embedding into the Borel subgroup $\widetilde{B}$ of $\mathrm{GL}_{2}(q)$. This is just $U$ extended by the maximal torus $\widetilde{H}_{1}$ of $\widetilde{G}$, isomorphic to a direct product of the Frobenius group $\mathbb{F}_{q}^{+} \cdot \mathbb{F}_{q}^{\times}$with a cyclic group $C_{q-1}$. Since the latter has character degrees 1 and $q-1$ only, all characters of $B$ except for the four characters of degree $(q-1) / 2$ extend to $\widetilde{B}$. Since these correspond to $\chi_{a}^{ \pm}, \chi_{b}^{ \pm}$, the bijection is equivariant with respect to diagonal automorphisms as well, establishing (4). Applying Lemma 15.2, we see that all characters of $B$ extend to their inertia subgroups in $\mathbf{N}_{\widehat{G}}(B)$

To verify condition (8), we lift the map $H_{1}^{*} \rightarrow\left\{x^{2}+2 x+s \mid s \in \mathbb{F}_{q}^{\times}\right\}$defined previously to a map

$$
\widetilde{H}_{1}^{*} \rightarrow\left\{x^{2}+a x+b \mid a, b \in \mathbb{F}_{q}^{\times}\right\}
$$

as follows. Elements of $\widetilde{H}_{1}^{*}$ are diagonal matrices; to $s=\operatorname{diag}\left(s_{1}, s_{2}\right)$ we associate the degree 2 polynomial $g_{s}=x^{2}+2 s_{1} x+s_{1} s_{2}$. This defines a bijection between elements of $\widetilde{H}_{1}^{*}$ and monic degree 2 polynomials over $\mathbb{F}_{q}$ with all coefficients non-zero. Note that this map really is a lift of our previous map. By interpreting $g_{s}$ as the characteristic polynomial of a semisimple element $t \in \widetilde{G}^{*}$, this allows to associate an irreducible character of $\widetilde{G}$ to $s$. Namely, if $t \notin \mathbf{Z}\left(\widetilde{G}^{*}\right)$ then we take the irreducible character $\pm \widetilde{R}_{i}(t)$, while if $t \in \mathbf{Z}\left(\widetilde{G}^{*}\right)$ we take the irreducible constituent of $\widetilde{R}_{i}(t)$ which is an extension of the principal character of $S$. Since the map preserves the constant coefficient of the characteristic polynomial, it preserves the restrictions to the center $\mathbf{Z}(\widetilde{G})$.

Now let $\lambda_{s} \in \operatorname{Irr}(B)$ indexed by $s \in H_{1}^{*}$ and $\iota\left(\lambda_{s}\right)=\chi_{t}$, where $t$ has characteristic polynomial $g=x^{2}+2 x+s$. Let $i$ be such that $t \in H_{i}^{*}$. Let $\widetilde{\theta}$ be the restriction to $\widetilde{G}$ of an extension of $\chi_{t}$ to $G(\theta)$. Then $\widetilde{\theta}$ is a Deligne-Lusztig character $\pm \widetilde{R}_{i}(\widetilde{t})$ for some preimage $\widetilde{t} \in \widetilde{H}_{i}^{*}$ of $t$ under the canonical epimorphism $\mathrm{GL}_{2}(q) \rightarrow \mathrm{PGL}_{2}(q)$. Denote by $\widetilde{g}=x^{2}+a x+b$ the characteristic polynomial of $\widetilde{t}$. So $s=4 b / a^{2}$, and $\widetilde{t}$ lies over the character $b$ of $\mathbf{Z}(\widetilde{G})$. Let $\widetilde{s}$ be the preimage of $\widetilde{g}$ under the map defined above, then the restriction of $\widetilde{s}$ to $H_{1}$ equals $s$. Thus $\widetilde{s}$ is an extension of $s$, lying over $b$ and invariant under the same field automorphisms as $\widetilde{\theta}$, so $(8)$ is satisfied.

For even $q$ the situation is simpler since $\mathbf{Z}(S)=1$, and we need not worry about central characters. This completes the proof in defining characteristic. 


\section{(15G) Exceptional multipliers.}

The only exceptional Schur multipliers for groups of the form $\operatorname{PSL}_{2}(q)$ are $C_{2}$ for $q=4$ and $C_{6}$ for $q=9$. But $2 \cdot \mathrm{PSL}_{2}(4) \cong \mathrm{SL}_{2}(5)$, and so all that remains is to consider the 3 -fold and 6 -fold covers of $\mathrm{PSL}_{2}(9) \cong A_{6}$. From the Atlas, one sees that $S=3 \cdot A_{6}$ has 10 faithful characters, with degrees $(3,3,3,3,6,6,9,9,15,15)$. Among the outer automorphisms of $A_{6}$ only the one called $2_{3}$ in the Atlas lifts to an automorphism of $S$ that centralizes $\mathbf{Z}(S)$. That automorphism fixes the faithful irreducible characters with degree exceeding 3 .

We need to consider the primes $p=5$ and $p=2$. For $p=5$, the Sylow normalizer in $S$ is isomorphic to $3 \times(5: 2)$, and in $S \cdot 2_{3}$, it is $3 \times(5: 4)$. The required bijection is easily established, and condition (8) holds because the relevant factor groups are cyclic, and so invariant characters extend. For $p=2$ the Sylow normalizer in $S$ is $3 \times \mathrm{D}_{8}$, and in $S \cdot 2_{3}$, it is $3 \times \mathrm{SD}_{16}$. (Here, $\mathrm{D}_{8}$ is dihedral of order 8 and $\mathrm{SD}_{16}$ is semidihedral of order 16.) Again it is easy to find the required bijection, and again condition (8) holds automatically.

For the group $S=6 \bullet A_{6}$, none of the outer automorphisms of $A_{6}$ lifts to an automorphism centralizing $\mathbf{Z}(S)$, and so conditions (4) and (8) will be automatically satisfied for any bijection. The group $S$ has eight faithful characters of degrees $(6,6,6,6,12,12,12,12)$, and we only need to consider $p=5$. Here the Sylow 5 -normalizer is $3 \times H$, where $H$ is a semidirect product of $C_{5}$ with $C_{4}$ such that $|\mathbf{Z}(H)|=2$. Clearly, this group also has eight irreducible characters faithful on the center. We have completed the proof of Theorem 15.3.

\section{The Suzuki simple groups are good.}

In this section we verify the conditions in Section 10 for the family of simple Suzuki groups $X=\operatorname{Sz}\left(q^{2}\right)$ with $q^{2}=2^{2 f+1}$, where $f \geq 1$. (We follow the usual convention, where $q$ is the square root of an odd power of 2, and hence is non-rational.) This group is its own universal covering group, except when $q^{2}=8$, where the Schur multiplier is a Klein four-group. We have $|X|=q^{4}\left(q^{2}-1\right)\left(q^{4}+1\right)$.

\section{(16A) The irreducible characters of $\mathrm{Sz}\left(q^{2}\right)$.}

We begin by recalling the parametrization of irreducible characters of $X=\operatorname{Sz}\left(q^{2}\right)$, due to Suzuki [20]. (See also Theorem XI.5.10 of [8].) This group has four unipotent characters $\chi_{1}, \ldots, \chi_{4}$. Their degrees are $\chi_{1}(1)=1, \chi_{2}(1)=\chi_{3}(1)=\sqrt{2} q\left(q^{2}-1\right) / 2$ and $\chi_{4}(1)=q^{4}$. The other irreducible characters are best regarded as Deligne-Lusztig characters indexed by suitable conjugacy classes of semisimple elements of $X$.

Let $H_{1}, H_{2}$ and $H_{3}$ denote representatives for the conjugacy classes of maximal tori of $X$, of orders $q^{2}-1, q^{2}-\sqrt{2} q+1$ and $q^{2}+\sqrt{2} q+1$, respectively. Their normalizers $N_{i}=\mathbf{N}_{X}\left(H_{i}\right)$ are Frobenius groups of order $2\left|H_{1}\right|, 4\left|H_{2}\right|, 4\left|H_{3}\right|$ respectively, and it follows that every nonidentity semisimple element of $X$ is conjugate either to two elements of $H_{1}$ or to four elements of $H_{2}$ or to four elements of $H_{3}$. (See [8] Theorem XI.3.10.) The nonunipotent irreducible characters of $X$, therefore, are indexed by the nonidentity elements $s \in H_{1} \cup H_{2} \cup H_{3}$ modulo conjugation in $X$, and we write $\chi_{s}$ for the character corresponding to $s$. We have that $\chi_{s}(1)=|X|_{2^{\prime}} /\left|H_{i}\right|$ if $s \in H_{i}$. See Table 3 for an overview.

TABLE 3. Irreducible characters of $\mathrm{Sz}\left(q^{2}\right)$. 


\begin{tabular}{|c|c|c|}
\hline$\theta$ & $\theta(1)$ & number \\
\hline$\chi_{1}$ & 1 & 1 \\
\hline$\chi_{2}, \chi_{3}$ & $q\left(q^{2}-1\right) / \sqrt{2}$ & 2 \\
\hline$\chi_{4}$ & $q^{4}$ & 1 \\
\hline$\chi_{s} \quad s \in H_{1}, s \neq 1$ & $q^{4}+1$ & $\left(q^{2}-2\right) / 2$ \\
\hline$\chi_{s} \quad s \in H_{2}, s \neq 1$ & $\left(q^{2}-1\right)\left(q^{2}+\sqrt{2} q+1\right)$ & $\left(q^{2}-\sqrt{2} q\right) / 4$ \\
\hline$\chi_{s} \quad s \in H_{3}, s \neq 1$ & $\left(q^{2}-1\right)\left(q^{2}-\sqrt{2} q+1\right)$ & $\left(q^{2}+\sqrt{2} q\right) / 4$ \\
\hline
\end{tabular}

We mention that Table 3 remains valid even in the degenerate case where $q^{2}=2$ and the group $X$ is the Frobenius group of order 20. In that case, the only characters $\chi_{s}$ that exist are those of the third type, where $1 \neq s \in H_{3}$ and $\left|H_{3}\right|=5$. There is just one such character in this situation

The group of outer automorphisms of $X$ is cyclic of (odd) order $2 f+1$, isomorphic to the group of field automorphisms of $\mathbb{F}_{q^{2}}$. A generator of $\operatorname{Out}(X)$ acts on $H_{1}$ by squaring. (This is the natural action on $H_{1} \cong \mathbb{F}_{q^{2}}^{\times}$.) On $H_{2}$ and $H_{3}$, it acts by sending elements to their $2^{4}=16$ th power, as can be seen by embedding them into $\mathbb{F}_{q^{8}}^{\times}$. In particular all inertia factor groups in $\operatorname{Aut}(X)$ of characters $\theta \in \operatorname{Irr}(X)$ are cyclic.

For $\theta \in \operatorname{Irr}(X)$ we set $G(\theta)=\operatorname{Aut}(X)_{\theta}$, the inertia group of $\theta$ in $\operatorname{Aut}(X)$. Since $|\mathbf{Z}(X)|=1$ this guarantees that conditions (5), (6) and (7) of Section 10 hold. Since the inertia factor groups are cyclic, (8) will automatically be satisfied for any bijection preserving inertia groups.

(16.1) THEOREM. Let $X=\operatorname{Sz}\left(q^{2}\right)$, where $q^{2}=2^{2 f+1} \geq 8$. Then $X$ is good for all prime divisors of its order.

Proof. We treat the various prime divisors of $|X|$ in separate subsections.

\section{(16B) Odd primes.}

First, let $p$ be a prime divisor of $q^{2}+\sqrt{2} q+1$, so that $H_{3}$ contains a Sylow $p$ subgroup $Q$ of $X$, and let $T=\mathbf{N}_{X}(Q)=\mathbf{N}_{X}\left(H_{3}\right)$. Then $T$ has four linear characters and $\left(q^{2}+\sqrt{2} q\right) / 4$ irreducible characters $\psi_{s}$ of degree 4 , indexed by the $T$-classes of nontrivial elements $s \in H_{3}$, and thus by nonidentity elements $s \in H_{3}$ modulo conjugation in $X$. We define

$$
\iota_{p}: \operatorname{Irr}_{p^{\prime}}(X) \rightarrow \operatorname{Irr}_{p^{\prime}}(T)=\operatorname{Irr}(T)
$$

by sending the four unipotent characters to the four linear characters of $T$, and $\chi_{s} \mapsto \psi_{s}$. Note that all other irreducible characters of $X$ have degree divisible by $q^{2}+\sqrt{2} q+1$, hence by $p$, and so this gives a bijection as in condition (3). It is immediate to verify that this bijection preserves degrees modulo $q^{2}+\sqrt{2} q+1$, hence modulo $p$. The unipotent characters of $X$ are invariant under $\operatorname{Aut}(X)$ and the linear characters of $T$ are invariant under $\mathbf{N}_{\mathrm{Aut}(X)}(Q)$, while the action on the $\chi_{s}$ is the same as on the $\psi_{s}$, so $\iota$ is equivariant with respect to $\mathbf{N}_{\operatorname{Aut}(X)}(Q)$, proving (4). Condition (8) holds because the relevant factor groups are cyclic, and this completes the proof for primes $p$ dividing $q^{2}+\sqrt{2} q+1$. 
The cases of primes $p$ dividing $\left(q^{2}-\sqrt{2} q+1\right)$ and $p$ dividing $\left(q^{2}-1\right)$ are very similar and hence are omitted. (In the latter case, only two of the unipotent characters have degree not divisible by $p$, and in this situation $T=\mathbf{N}_{X}\left(H_{1}\right)$ is dihedral of order $2\left|H_{1}\right|$, and so has just two linear characters.)

(16C) The defining prime $p=2$.

Let $Q$ be a Sylow 2-subgroup of $X$. The structure of $Q$ is described in [8] Section XI.3, for example. In particular, the commutator factor group is isomorphic to $\mathbb{F}_{q^{2}}^{+}$, and so is elementary abelian of order $q^{2}$. The normalizer $B=\mathbf{N}_{X}(Q)$ is a Borel subgroup of $X$; it is a split extension of $Q$ with a cyclic group of order $q^{2}-1$ acting regularly on the nonidentity elements of $Q / Q^{\prime}$. (See Lemma XI.5.9 of [8].) Thus $\operatorname{Irr}_{p^{\prime}}(B)$ consists of $q^{2}-1$ linear characters $\psi_{t}$, indexed by elements $t \in \mathbb{F}_{q^{2}}^{\times}$and one character $\psi_{0}$ of degree $q^{2}-1$. We choose $T=B$, so (1) and (2) are satisfied, and we define

$$
\iota: \operatorname{Irr}_{p^{\prime}}(X) \rightarrow \operatorname{Irr}_{p^{\prime}}(T)
$$

by sending $\chi_{1}$ to $\psi_{0}$ and the $\chi_{s}$ to the $\psi_{t}$ as follows.

First, observe from Table 3 that the total number of characters $\chi_{s}$ (of odd degree) of $X$ is $q^{2}-1$, and this is the same as the number of characters $\psi_{t}$ of $B$. To prove that there exists a $\mathbf{N}_{\text {Aut }(X)}(T)$-equivariant bijection $\iota$, it suffices to show that each automorphism in $\mathbf{N}_{\text {Aut }(X)}(T)$ fixes equal numbers of characters $\chi_{s}$ and $\psi_{t}$. It suffices to consider outer automorphisms, which we can identify with automorphisms of the field $\mathbb{F}_{q^{2}}$. We must show then, that each field automorphism $\gamma$ fixes equal numbers of characters $\chi_{t}$ of $X$ and $\psi_{t}$ of $B$.

The $\psi_{t}$ are indexed by nonzero field elements $t$, and $\gamma$ fixes $\psi_{t}$ precisely when $\gamma$ fixes $t$, and so the number of these is $|K|-1$, where $K \subseteq \mathbb{F}_{q^{2}}$ is the fixed field of $\gamma$. To count the characters $\chi_{s}$ that are fixed by $\gamma$, consider the corresponding Suzuki group $X(K)$, viewed as a subgroup of our group $X=X\left(\mathbb{F}_{q^{2}}\right)$. (If $|K|=2$, then $X(K)$ is not simple, but that will not affect our count.) The characters $\chi_{s}$ that are fixed by $\gamma$ are exactly those whose index $s$ lies in the subgroup $X(K)$, and we have already seen that the number of these is exactly $|K|-1$, as required. This shows that the map $\iota$ can be constructed to be $\mathbf{N}_{\text {Aut }(X)}(T)$-equivariant, as needed. This establishes condition (4), and as before, condition (8) holds because the relevant factor groups are cyclic. This completes the proof of Theorem 16.1 except in the case $q^{2}=8$, where there is an exceptional Schur multiplier.

\section{(16D) The exceptional multiplier.}

The only exceptional Schur multiplier for groups $\mathrm{Sz}\left(q^{2}\right)$ is the Klein group when $q=8$, and so it remains to consider 2 -fold covers of $\mathrm{Sz}(8)$. Note that all three of them are conjugate under the outer automorphism of order 3 , hence it suffices to treat just one such cover. From the Atlas one sees that $S=2 \cdot \mathrm{Sz}(8)$ has eight faithful characters, with degrees $(40,40,40,56,56,56,64,104)$. Since $\operatorname{Out}(S)$ is trivial in this case, we need not worry about outer automorphisms. The relevant primes are $p=5,7,13$, and in all three cases, the Sylow normalizer $T=\mathbf{N}_{S}(Q)$ in $S$ is isomorphic to the direct product of the normalizer in $X=\mathrm{Sz}(8)$ with the central subgroup of order 2. A quick check shows that there is a bijection $\operatorname{Irr}_{p^{\prime}}(S) \rightarrow \operatorname{Irr}_{p^{\prime}}(T)$ preserving degrees mod $p$ in all three cases. This completes the proof of Theorem 16.1. 


\section{The Ree simple groups $\mathrm{R}\left(q^{2}\right)$ are good.}

In this section we verify the conditions in Section 10 for the family of simple Ree groups $X=\mathrm{R}\left(q^{2}\right)={ }^{2} \mathrm{G}_{2}\left(q^{2}\right)$, where $q^{2}=3^{2 f+1}$ with $f \geq 1$. (Thus $q$ is the square root of an odd power of 3.) The group $\mathrm{R}(3)$ is not simple; it is isomorphic to $\operatorname{Aut}\left(\mathrm{PSL}_{2}(8)\right)$ which was considered before. These groups are their own universal covering groups, and we have $|X|=q^{6}\left(q^{2}-1\right)\left(q^{6}+1\right)$.

The irreducible complex characters of $\mathrm{R}\left(q^{2}\right)$ were determined by Ward [22]. A convenient parametrization is obtained via Lusztig's Jordan decomposition of characters by semisimple conjugacy classes of $X$. The trivial class gives rise to the eight unipotent characters $\chi_{1}, \ldots, \chi_{8}$ and the unique class of involutions gives rise to two characters $\chi_{a}, \chi_{a}^{\prime}$. Each remaining semisimple element is regular, and so lies in a unique maximal torus of $X$, and each of these classes parametrizes a single irreducible Deligne-Lusztig character. There are four conjugacy classes of maximal tori, with representatives $H_{1}$ of order $q^{2}-1, H_{2}$ of order $q^{2}+1, H_{3}$ of order $q^{2}-\sqrt{3} q+1$ and $H_{4}$ of order $q^{2}+\sqrt{3} q+1$. These have indices 2, 6, 6 and 6 in their respective normalizers, and hence a regular semisimple element in $H_{i}$ lies in a conjugacy class of length 2,6,6 and 6 respectively in $\mathbf{N}_{X}\left(H_{i}\right)$. While $H_{1}, H_{3}$ and $H_{4}$ are cyclic, $H_{2}$ is a direct product $C_{\left(q^{2}+1\right) / 2} \times C_{2}$.

We collect the degrees and numbers of characters in Table 4.

TABLE 4. Irreducible characters of $\mathrm{R}\left(q^{2}\right)$.

\begin{tabular}{|c|c|c|}
\hline$\theta$ & $\theta(1)$ & number \\
\hline$\chi_{1}$ & 1 & 1 \\
\hline$\chi_{2}, \chi_{3}$ & $q\left(q^{4}-1\right) / \sqrt{3}$ & 2 \\
\hline$\chi_{4}, \chi_{5}$ & $q\left(q^{2}-1\right)\left(q^{2}-\sqrt{3} q+1\right) /(2 \sqrt{3})$ & 2 \\
\hline$\chi_{6}, \chi_{7}$ & $q\left(q^{2}-1\right)\left(q^{2}+\sqrt{3} q+1\right) /(2 \sqrt{3})$ & 2 \\
\hline$\chi_{8}$ & $q^{6}$ & 1 \\
\hline$\chi_{a}$ & $q^{4}-q^{2}+1$ & 1 \\
\hline$\chi_{a}^{\prime}$ & $q^{2}\left(q^{4}-q^{2}+1\right)$ & 1 \\
\hline$\chi_{s} \quad s \in H_{1}, s^{2} \neq 1$ & $q^{6}+1$ & $\left(q^{2}-3\right) / 2$ \\
\hline$\chi_{s} \quad s \in H_{2}, s^{2} \neq 1$ & $\left(q^{2}-1\right)\left(q^{4}-q^{2}+1\right)$ & $\left(q^{2}-3\right) / 6$ \\
\hline$\chi_{s} \quad s \in H_{3}, s \neq 1$ & $\left(q^{4}-1\right)\left(q^{2}+\sqrt{3} q+1\right)$ & $\left(q^{2}-\sqrt{3} q\right) / 6$ \\
\hline$\chi_{s} \quad s \in H_{4}, s \neq 1$ & $\left(q^{4}-1\right)\left(q^{2}-\sqrt{3} q+1\right)$ & $\left(q^{2}+\sqrt{3} q\right) / 6$ \\
\hline
\end{tabular}

The group of outer automorphisms of $X$ is cyclic of (odd) order $2 f+1$, isomorphic to $\operatorname{Aut}\left(\mathbb{F}_{q^{2}}\right)$. A generator of $\operatorname{Out}(X)$ acts naturally on $H_{1} \cong \mathbb{F}_{q^{2}}^{\times}$. On $H_{2}$ it acts by sending elements to their 9 th power, and on $H_{3}$ and $H_{4}$ by sending elements to their $3^{6}=729$ th power, as can be seen by embedding these tori into $\mathbb{F}_{q^{12}}^{\times}$. In particular all inertia factor groups in $\operatorname{Aut}(X)$ of characters $\theta \in \operatorname{Irr}(X)$ are cyclic.

(17.1) THEOREM. Let $X=\mathrm{R}\left(q^{2}\right)$, where $q^{2}=3^{2 f+1} \geq 27$. Then $X$ is good for all prime divisors of its order. 
Proof. First, we consider primes $p$ dividing $\left(q^{2}+\sqrt{3} q+1\right)$. Then the six unipotent characters $\chi_{1}, \ldots, \chi_{5}$ and $\chi_{8}$ and also another $\left(q^{2}+\sqrt{3} q\right) / 6$ Deligne-Lusztig characters are of $p^{\prime}$-degree. A Sylow $p$-subgroup is contained in $H_{4}$, and its normalizer is $T=\mathbf{N}_{X}\left(H_{4}\right)$. Since $T / H_{4}$ acts semiregularly on the nonidentity elements of $H_{4}$, we see that $\operatorname{Irr}(T)$ has six linear characters and $\left(q^{2}+\sqrt{3} q\right) / 6$ irreducible characters of degree 6 , and there is an obvious bijection $\iota: \operatorname{Irr}_{p^{\prime}}(X) \rightarrow \operatorname{Irr}_{p^{\prime}}(T)$. This is compatible with the action of field automorphisms on both sets. Exactly the same arguments apply to primes $p$ dividing $\left(q^{2}-\sqrt{3} q+1\right)$.

Now let $p$ be an odd prime divisor of $\left(q^{2}-1\right)$. Then $\chi_{1}, \chi_{8}, \chi_{a}, \chi_{a}^{\prime}$ and a further $\left(q^{2}-3\right) / 2$ characters of $X$ have $p^{\prime}$-degree. The normalizer $T=\mathbf{N}_{X}\left(H_{1}\right)$ is the normalizer of a Sylow $p$-subgroup of $X$. It acts on $H_{1}$ by inversion, and so precisely two characters in $\operatorname{Irr}\left(H_{1}\right)$ remain invariant, while the others are interchanged in pairs. This gives four linear characters and $\left(q^{2}-3\right) / 2$ characters of degree 2 in $\operatorname{Irr}(T)$. Again, the obvious bijection is equivariant with respect to field automorphisms.

Next, let $p$ be an odd prime divisor of $\left(q^{2}+1\right)$. Here $\operatorname{Irr}_{p^{\prime}}(X)$ contains the eight characters $\chi_{1}, \chi_{4}, \ldots, \chi_{8}$ and $\chi_{a}$ and $\chi_{a}^{\prime}$ and also $\left(q^{2}-3\right) / 6$ Deligne-Lusztig characters $\chi_{s}$. The normalizer $T=\mathbf{N}_{X}\left(H_{2}\right)$ of $H_{2}$ is the normalizer of a Sylow $p$-subgroup of $X$, and it acts semiregularly on the set of characters of $H_{2}$ of order larger than 2 , giving $\left(q^{2}-3\right) / 6$ characters of degree 6 in $\operatorname{Irr}(T)$. The characters of order 2 of $H_{2}$ are permuted transitively, yielding two characters of degree 3 , and the trivial character of $H_{2}$ has six extensions to $T$. Again, the desired bijection is obvious.

For $p=2$, we see that $\operatorname{Irr}_{p^{\prime}}(X)$ consists of $\chi_{1}, \chi_{4}, \ldots, \chi_{8}$ and $\chi_{a}$ and $\chi_{a}^{\prime}$. A Sylow 2 -subgroup $Q$ of $X$ is elementary abelian of order 8 , with normalizer quotient a Frobenius group of order 21. Thus the seven non-trivial characters of $Q$ give rise to three characters of degree 7 of the normalizer $T=\mathbf{N}_{X}(Q)$, while the trivial character has three extensions and gives rise to two further characters of degree 3 . Since $T$ is already contained in the group $\mathrm{R}(3)=\operatorname{Aut}\left(\mathrm{PSL}_{2}(8)\right)$ over the prime field, all of these characters are invariant under all field automorphisms, and so any bijection from $\operatorname{Irr}_{p^{\prime}}(X)$ to $\operatorname{Irr}_{p^{\prime}}(T)$ will do.

For $p=3$, the irreducible characters of $p^{\prime}$-degree are the semisimple characters. These are $\chi_{1}, \chi_{a}$ and the Deligne-Lusztig characters $\chi_{s}$. The unipotent radical $U$ of a Borel subgroup $B$ is a Sylow 3-subgroup of $X$, and $B$, its normalizer, is a split extension of $U$ by the maximal torus $H_{1}$ acting regularly on $U / U^{\prime}$. Thus $B$ has $q^{2}-1$ linear characters and one character of degree $q^{2}-1$. (See Lemma 5 of [5].) In this situation, the existence of an $\mathbf{N}_{\text {Aut }(X)}(B)$-equivariant bijection was established by Eaton in the course of proving Dade's invariant conjecture for these groups. (See Theorem 8 of [5].) This completes the proof of Theorem 17.1.

We conclude by noting that in all cases, the bijections constructed above satisfy congruences for the character degrees as proposed in [11]. 


\section{REFERENCES}

1. J. L. Alperin, "The main problem of block theory" Proceedings of the Conference on Finite Groups (Park City, Utah, 1975), 341-356. Academic Press, New York, 1976.

2. R. W. Carter, Finite Groups of Lie Type, Wiley-Interscience, New York, 1985.

3. E. C. Dade, "Counting characters in blocks I" Inventiones Mathematicae 109 (1992), $187-210$.

4. E. C. Dade, "Counting characters in blocks II" J. Reine Angew. Math. 448 (1994), 97-190.

5. C. Eaton, "Dade's inductive conjecture for the Ree groups of type $G_{2}$ in the defining characteristic", J. of Algebra 226 (2000), 614-620.

6. C. W. Eaton and G. R. Robinson, "On a minimal counterexample to Dade's projective conjecture", J. Algebra 249 (2002), no. 2, 453-462.

7. J. A. Green, G. I. Lehrer and G. Lusztig, "On the degrees of certain group characters", Quart. J. Math. Oxford Ser. 227 (1976), 1-4.

8. B. Huppert and N. Blackburn, Finite groups. III. Grundlehren der Mathematischen Wissenschaften 243 Springer-Verlag, Berlin-New York, 1982.

9. I. M. Isaacs, Character theory of finite groups Dover, New York, 1994.

10. I. M. Isaacs Algebra: a graduate course Brooks-Cole, Pacific Grove, 1994.

11. I. M. Isaacs and Gabriel Navarro, "New refinements of the McKay conjecture for arbitrary finite groups" Ann. of Math. (2) 156 (2002), 333-344.

12. G. Lusztig, "On the representations of reductive groups with disconnected centre", pp. 157-166 in: Orbites unipotentes et représentations, I. Astérisque 168, (1988).

13. G. Malle, "The inductive McKay condition for simple groups not of Lie type", to appear in Comm. Algebra.

14. G. Malle, "Height 0 characters of finite groups of Lie type", Submitted 2006.

15. M. Murai, "A remark on the Alperin-Mckay conjecture." J. Math. Kyoto Univ. 44 (2004), 245-254.

16. G. Navarro, "The McKay conjecture and Galois automorphisms", Ann of Math. 160 (2004), 1129-1140.

17. T. Okuyama and M. Wajima, "Character correspondence and $p$-blocks of $p$-solvable groups", Osaka J. Math. 17 (1980), 801-806.

18. G. R. Robinson, "Dade's projective conjecture for p-solvable groups", J. Algebra 229 (2000), 234-248.

19. B. Späth, "Die McKay Vermutung für quasi-einfach Gruppen vom Lie-Typ", Doctoral thesis, Technische Universität Kaiserslautern, 2007. 
20. M. Suzuki, "On a class of doubly transitive groups", Ann. of Math. 75 (1962), 105-145.

21. A. Turull, "Strengthening the McKay conjecture to include local fields and local Schur indices", To appear.

22. H. N. Ward, "On Ree's series of simple groups", Trans. Amer. Math. Soc. 121 (1966), $62-89$.

23. J. H. Walter, "The characterization of finite groups with abelian Sylow 2-subgroups", Ann. of Math. 89 (1969), 405-514.

24. R. A. Wilson, "The McKay conjecture is true for the sporadic simple groups", $J$. Algebra 207 (1998), 294-305.

24. T. R. Wolf, "Variations on McKay's character degree conjecture" J. Algebra 135 (1990), 123-138. 